\title{
Optimization of Variational Boussinesq Models
}

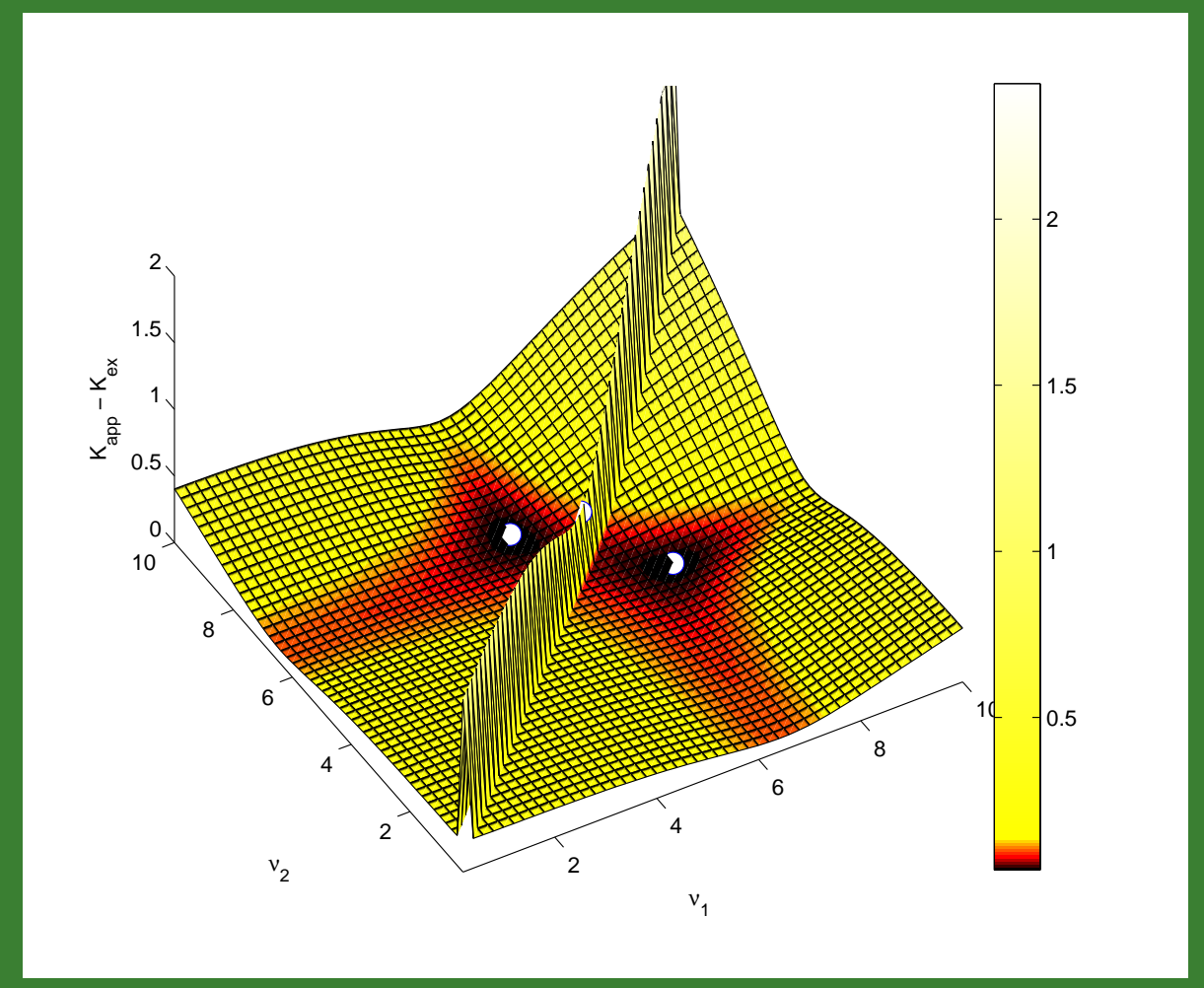

IVAN LAKHTUROV 
The research described in this thesis was undertaken at the group of Applied Analysis and Mathematical Physics (AAMP), University of Twente, Enschede, The Netherlands.

The research was partially done at the research institute LabMathIndonesia, Bandung, Indonesia.

The research was supported by Netherlands Science Foundation NWOSTW, TWI-7216 and by KNAW (Royal Netherlands Academy of Arts and Sciences).

Ivan Lakhturov: Optimization of

Variational Boussinesq Models (c) 2012

Printed by Wöhrmann Printing Service, Zutphen, The Netherlands.

ISBN: 978-90-365-3447-5

Front cover figure: see Fig. 21 and Chapter 4.

Back cover figure: see Fig. 18 and Chapter 3 .

This thesis was typeset using LYX \& CLAssicthesis. 


\section{OPTIMIZATION OF \\ VARIATIONAL BOUSSINESQ MODELS}

\section{PROEFSCHRIFT}

ter verkrijging van

de graad van doctor aan de Universiteit Twente,

op gezag van de rector magnificus,

prof. dr. H. Brinksma,

volgens besluit van het College voor Promoties

in het openbaar te verdedigen

op vrijdag 9 november 2012 om 12.45 uur

door

Ivan Lakhturov

geboren op 30 september 1982

te Charkov, Oekraïne 
Dit proefschrift is goedgekeurd door de promotor prof. dr. ir. E.W.C. van Groesen 
To the loving memory of Zoya, the brave World War II soldier and my caring grandmother 

The research presented in this thesis is concentrated mostly around the properties of the Variational Boussinesq Model (VBM). The VBM is a model for waves above a layer of ideal fluid, which conserves mass, momentum, energy, and has decreased dimensionality compared to the full problem. It is derived from the Hamiltonian formulation via an approximation of the kinetic energy, and can provide approximate dispersion characteristics. In particular, dispersive properties of a model are important for a large number of practical applications, especially if modelled waves have a broad spectrum.

The most important assessment for a model is a comparison between results obtained from the model and experimental data. We show how well the VBM performs in a few complex cases, such as focussing wave groups with broad spectra. The real-life experiments with this type of waves were performed at the facilities of the MARIN hydrodynamic laboratory (Maritime Research Institute Netherlands). In order to put the work into the context of existing research, we compare the obtained results to those of other models, in particular the AB-equation which is briefly discussed in the thesis as well.

Having free parameters in the VBM gives opportunities to optimize the parameters depending on the specifcs of the application. We explore possibilities of such optimization, interchanging norms in different optimization criteria. Then the question rises, which of these norms is the best? We come up with the novel kinetic energy optimization criterium that is natural for the VBM and gives seemingly the best result in the considered test cases.

Another important property from the practical viewpoint is how well a model simulates reflection. We study the reflective properties of the VBM and compare them to previous results by other authors. We also derive and investigate an analytical reflection model of the WKB (Wentzel-Kramers-Brillouin) type, whose performance is surprisingly good.

For the numerical implementation of the VBM we employ a Finite Element Method (FEM), and a pseudo-spectral method in case of the AB-equation. In the thesis we concentrate on errors caused by the modelling process, and provide details of the numerical implementation in the first chapters and in the Appendix. This includes an embedded influx condition, which we use in signalling problems for both the AB-equation and VBM. 



\section{SAMENVATTING}

Het onderzoek van dit proefschrift concentreert zich voornamelijk op eigenschappen van Variationele Boussinesq modellen (VBM). Dat zijn modellen voor golven op een laag van een ideale vloeistof, die massa, impuls en energie-behoudend zijn, en een dimensiereductie hebben ten opzichte van de volledige formulering. Afgeleid uit de Hamiltonse formulering door een benadering van de kinetische energie, kunnen verschillende benaderingen van de dispersie-eigenschappen verkregen worden. Deze dispersie-eigenschappen zijn in het bijzonder van belang voor meerdere toepassingen, in het bijzonder als de golven een breed spectrum hebben.

De belangrijkste beoordeling van een model wordt verkregen door vergelijking van de modelresultaten met experimentele data. Wij tonen aan hoe goed de VBM presteert in enkele moeilijke gevallen, zoals focussing golfgroepen met breed spectra. De experimenten voor dit soort golven werden uitgevoerd door het hydrodynamische laboratorium MARIN (Maritime Research Institute Netherlands). Om het werk een plaats te geven in het huidige onderzoek vergelijken we de verkregen resultaten ook met die van andere modellen, in het bijzonder met de $\mathrm{AB}$-vergelijking die ook kort beschreven wordt.

De vrije parameters die in VBM beschikbaar zijn maken het mogelijk de parameters optimaal te kiezen, afhankelijk van de specifieke toepassing. We onderzoeken die optimale keuzen, in normen die corresponderen met verschillende optimaliteitscriteria. Maar dan dient de vraag zich aan welke keuze de beste is. Wij presenteren een nieuw optimaliteitscriterium dat gebaseerd is op de kinetische energie, hetgeen op natuurlijke wijze past bij VBM en dat ook de beste prestaties geeft in de beschouwde testcases.

Een andere belangrijke eigenschap is hoe goed een model reflecties kan simuleren. Wij bestuderen de reflectie-eigenschappen van VBM en vergelijken die met eerdere resultaten van andere auteurs. Wij leiden ook een analytisch WKB (Wentzel-Kramers-Brillouin) reflectiemodel af dat verbazingwekkend nauwkeurig is.

Voor de numerieke implementatie van de VBM gebruiken we de Finite Element Methode (FEM), en voor de AB-vergelijking een pseudospectrale methode. Met concentratie op het modelleerproces, geven we details van de numerieke implementatie in de eerste hoofdstuken en in de Appendix, inclusief een beschrijving van een embedded influxmethode die gebruikt wordt in de signaalproblemen voor de ABvergelijking en de VBM. 

The research presented in this thesis is based on a few years of efforts, exerted within the group of Applied Analysis and Mathematical Physics, University of Twente, Enschede, The Netherlands. I greatly enjoyed these years, and would like to express my deep gratitude to my supervisor, professor Brenny van Groesen, for guidance on the way and patience while waiting for me to deliver results. Part of the work was done at the research institute LabMath-Indonesia, Bandung, Indonesia. The support of its director Aan Andonowati is also cordially appreciated. The funding and support provided by Netherlands Science Foundation NWO-STW (TWI-7216) and KNAW (Royal Netherlands Academy of Arts and Sciences) gave me an opportunity to perform this research. The use of MARIN (Maritime Research Institute, Netherlands) experimental data is highly appreciated, as well.

My appreciation goes to my co-authors Liam Lie, Didit Adytia and Wenny Kristina, and to all staff members and PhD-students of both AAMP and NACM groups of the University of Twente, who shared working hours and coffee breaks with me: Natanael Karjanto, Tim Op 't Root, Milan Maksimović, Alyona Ivanova, Sid Visser, Marcel Lourens, Sena Sopaheluwakan, Vijaya Raghav Ambati, Bob Peeters, David Lopez Penha, Henk Sollie, Domokos Sármány, Lilya Ghazaryan, Elena Gagarina, Svetlana Polenkova, Shavarsh Nurijanyan, Stephan van Gils, Manfred Hammer, Gerard Jeurnink, Antonios Zagaris, Hil Meijer, Onno Bokhove, Jaap van der Vegt, Adri van der Meer. Special thanks to our secretaries, Marielle Slotboom-Plekenpol, Carin KrijnenSmid and Linda Wychgel-van Dalm. I appreciate the help of LabMathIndonesia students Dwi Fajar Saputri, Zulkarnain and Amanda P. Rudiawan, who performed the simulations for one of the subsections in the thesis. And a separate word of thanks to Gert Klopman and Mike Botchev, for infrequent but valuable discussions.

And many thanks to my friends: Sander Rhebergen, René Redder, Alexander Shpak, Andrey Khrustaleu, Andrey Sazonov, Konstantin Laevsky, Dmitriy Kirichenko, Evgeniy Dobrinov, Sergey Amstibovskiy, and my first teacher, Vyacheslav Epshtein. Finally, my sincere gratitude is to my parents and relatives, including my sister, the family of my brother, Slivko family, and personally to Oya Tagit. Thank you for supporting me during these years. 

CONTENTS

SUMMARY vii

SAMENVATTING viii

ACKNOWLEDGMENTS X X

1 INTRODUCTION

1.1 Motivation ................. I

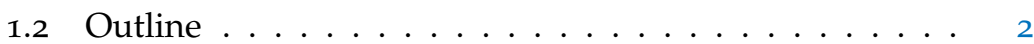

2 WAVE MODELLING 7

2.1 Variational Boussinesq Model . . . . . . . . . . . 7

2.1.1 Linear Shallow Water Equation . . . . . . . . . . . 9

2.1.2 VBM with one profile ........... 10

2.1.3 VBM with multiple profiles ......... 12

2.1.4 Numerical implementation ........... 12

2.1.5 Dispersion of the model . . . . . . . . . . 14

2.1.6 Advantages of VBM . . . . . . . . . . . 16

2.2 Uni-directional AB-equation . . . . . . . . . . . . . . 17

2.2.1 Derivation of AB-equation . . . . . . . . 18

2.2.2 Numerical implementation ........... 22

2.2.3 Highest Stokes wave test case . . . . . . . . . . . 24

2.3 Influx in signalling problem . . . . . . . . . . . . . . 27

2.4 Conclusions and remarks ............ 31

3 OPTIMIZATION OF VBM WITH ONE PROFILE 37

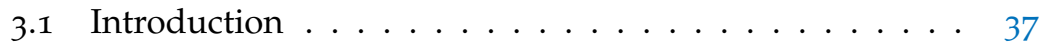

3.2 The VBM dispersion relations . . . . . . . . . . . 40

3.2.1 VBM dispersion relation ........... 41

3.2.2 Parabolic Approximation ........... 43

3.2.3 Hyperbolic Cosine Approximation . . . . . . . . 43

3.3 Optimization criteria ... . . . . . . . . . . 44

3.3.1 Weighted least square formulations . . . . . . 47

3.3.2 Kinetic energy optimization criteria . . . . . . 48

3.4 Test cases . . . . . . . . . . . . . . . . . . . 49

3.4.1 Focussing wave groups ........... 49

3.4.2 Accurate simulation of the focussing process . . 50

3.5 Optimized VBM simulations . . . . . . . . . 52

3.5.1 Calculation of optimal values . . . . . . . . 52

3.5 .2 Signal at focussing point ............. 53

3.5.3 Maximal wave heights comparisons . . . . . 56 
3.6 Conclusions and remarks . . . . . . . . . 57

4 OPtimized VBM With MUltiple PROFiles 65

4.1 Introduction . . . . . . . . . . . . . 65

4.2 Variational Boussinesq Model with multiple profiles . . 68

4.2.1 Hamiltonian evolution equations . . . . . . . . 68

4.2.2 Dispersion relation ............. 69

4.2.3 Numerical implementation ......... 72

4.3 Optimization criterion ............. 73

4.3.1 Kinetic energy optimization criterion ..... 73

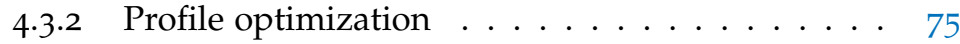

4.4 Test case . . . . . . . . . . . . . . 78

4.4.1 Focussing wave group (FWG) . . . . . . . 78

4.4.2 Accurate simulation of the focussing process . . 79

4.5 Optimized VBM simulations . . . . . . . . . . . . 80

4.5.1 Signal at focussing point ........... 81

4.5 .2 Maximal temporal amplitudes ........ 81

4.5.3 Simulation results with other codes ...... 82

4.6 Conclusions and remarks ............ 83

5 REFLECTION IN VBM AND WKB 91

5.1 Introduction ................... 91

5.1.1 Reflection from a step in the bathymetry . . . . 92

5.1.2 Classical WKB-approximation ... . . . . . 94

5.2 Reflection-capable WKB-approximation . . . . . . . 95

5.3 Numerical experiment and reflection curve . . . . . . . . . 98

5.3.1 Setup of the experiment . . . . . . . . . . 99

5.3.2 Reflection in WKB-approximation . . . . . . . . 99

$5 \cdot 3 \cdot 3$ Reflection in VBM .............. 102

5.4 Conclusions and remarks .............. 103

6 CONCLUSIONS AND RECOMMENDATIONS 107

7 APPENDIX 111

7.1 Values of coefficients $\alpha, \beta$ and $\gamma \ldots \ldots$. . . . . III

7.2 Matrices in VBM numerical implementation . . . . . . 112

7.3 JONSWAP spectrum and kinetic energy minimization . 113 


\subsection{MOTIVATION}

Our main research interest lies in building wave models that can in a deterministic way predict appearance of oceanic rogue waves (also called freak waves and sometimes referred as giant or extreme waves). The rogue wave is a wave that is much higher than expected for the sea state. The common definition requires them to be at least twice as large as the significant wave height. Such waves can cause severe damages and lead to lethal outcomes. For example, between 1969 and 1994 at least 22 supercarriers were lost because of rogue waves [6]. There are evidences of ships, oil platforms and coastal structures, which were hit by them, sometimes with devastating results. Death toll of freak waves during the last half-century is measured in hundreds, if not thousands. A good review of the literature and phenomenology of rogue waves one can find in [3].

Rogue waves are studied mainly from two theoretical points of view. The first is the statistical study. After realizing that freak waves are not some mystical creatures appearing only in the marine folklore, but vice-versa, they seem to be responsible for a large number of ship wrecks, the statistical research obtained proofs that the probability of their appearance was seriously underestimated before. Observations obtained with wave gauges, buoys and other modern wave measuring devices give empirical confirmations to this [3].

Our approach lies in the stream of the other type of freak waves research, deterministic one. It is important to address not only the probabilistic questions like how often these waves appear. The questions of shape and other properties of such waves are also vital. If we can reproduce the rogue waves with our models and in a hydrodynamic laboratory, it will allow for further study, in particular, the research of influence that these waves can exert upon ships and open-sea and coastal structures.

It is believed [6] that three physical mechanisms can lead to appearance of large waves in a moderate wave field: spatial focusing (including refractive focusing due to bathymetry and current-wave interactions), dispersive focusing, and nonlinear focusing. Dispersive focusing is the main topic we study throughout this thesis. Generally, the theoretical, numerical and experimental research of rogue waves becomes more and more topical and obtains larger momentum with time. A particular boost in this research is observed since 2000. 

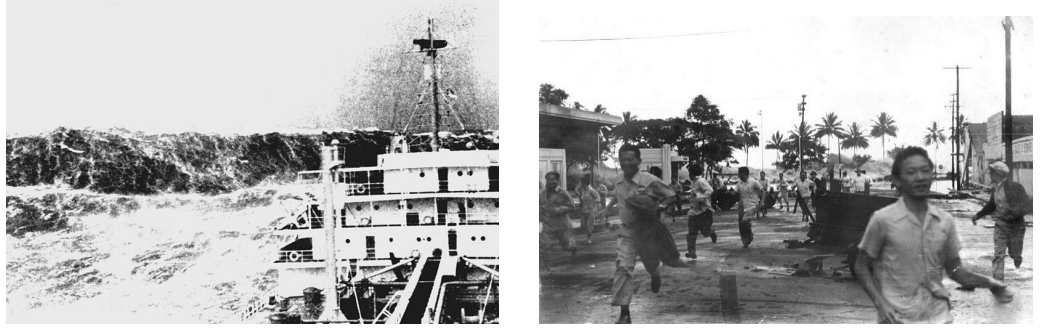

Figure 1: At the left a freak wave approaches the ship in the Bay of Biscay. At the right residents of Hawaii run from the approaching tsunami. Image courtesy of Wikipedia.

The human toll of tsunamis is far greater than one caused by rogue waves. As a result of the most devastating tsunami in human history, happened on 26 December 2004, more than 230,000 people died in the Asian region. The material damage brought by it is hardly countable.

The importance of the research in this area is indisputable, and a lot of efforts are being spent to improve tsunami models in all phases of the process: tsunami generation, propagation, shoaling and runup [4]. Generation usually happens due to an earthquake, but some tsunamis arise also as a result of a landslide, volcanic eruption, comet or asteroid impact [2]. And depending on its type, the generation may require very different modelling approaches. For the (local) simulation of the propagation phase, the shallow water equation and similar models are usually considered as satisfactory, but nowadays, with global tsunami simulations becoming a commonplace, it becomes necessary to incorporate tides and Coriolis forces into the models.

As regards tsunami shoaling and runup process, an appropriately accurate and fast prediction by models is challenging, and of the vital importance. With recent developments of tsunami real-time warning systems, the models' performance has potential of saving thousands of human lives. It is also promising to make use of such models, which realistically incorporate e. g. both tsunami propagation and shoaling processes, instead of coupling different models together.

Harbour simulations are of a considerable interest as well. One example of questions that rise in this regard is whether resonant (standing) waves will appear in particular harbour geometry, and if the answer is yes, how to improve artificially the harbour coastline for better protection?

\subsection{OUTLINE}

The outline of the thesis is as follows. 
In the next Chapter 2 we introduce the Variational Boussinesq Model (VBM), proceeding from its 'classical' form with the parabolic vertical dispersion profile, through the cosh VBM with one vertical profile and to the cosh VBM with multiple vertical profiles. The VBM will be used in every of the subsequent chapters, with the same approach of the model's complexity increase. We find it convenient to discuss advantages of the VBM in the same chapter.

It should be remarked that the analysis in this thesis is generally concentrated on the errors caused by the modelling process, and not on numerical accuracy errors. For the VBM simulations everywhere we used a FE-implementation with sufficiently fine grid, and we provide only the major numerical scheme, leaving other minor details to the Appendix. In addition, we describe an embedded boundary condition, which we use for the solution of signalling problems for both $\mathrm{AB}$-equation and the VBM.

The AB-equation serves as the main vehicle for purposes of comparison of the VBM to the auxiliary model. It is briefly introduced in the same chapter. The results of the AB-equation simulations are presented, for the test case with the so-called highest Stokes wave, together with concise description of the pseudo-spectral implementation. The demonstrated test case is taken from the published article [5].

In Chapter 3, having in mind a signalling problem, we search for optimal dispersive properties of the 1-D linear VBM with one vertical profile (over flat bottom) and, using finite element and (pseudo-) spectral numerical codes, investigate its quality. For the optimization we restrict to the class of potentials with hyperbolic vertical profiles that are parametrized by the wavenumber. The optimal wavenumber is obtained by minimizing the kinetic energy for the given signal and produces good results for two realistic test cases. Besides this kinetic energy principle we also consider various ad-hoc least square type of minimization problems for the error of the phase or group velocity. The test cases are two examples of focussing wave groups with broad spectra for which accurate experimental data are available from MARIN hydrodynamic laboratory [10]. To determine the quality of an 'optimized' wavenumber for the governing dynamics, we use accurate numerical simulations with the AB-equation to compare with VBM calculations for the whole range of possible wavenumbers. The comparison includes the errors in the signal at the focussing position, as well as the integrated errors of maximal and minimal wave heights along a spatial and temporal interval that is symmetric around the focussing event.

We observe that the performance of the cosh VBM with one vertical profile does not reach accuracy of the AB-equation. In particular we show that for any chosen parameter, all sufficiently short waves have 
the same, finite propagation speed. This erroneous behaviour is an inevitable consequence of the way we represent the fluid potential.

So, in Chapter 4 we take one of the considered test cases (the most extreme case of the focussing wave group) and use it for testing the derived generalization of the VBM, where the fluid potential is approximated not with one, but with a superposition of many vertical coshdispersion profiles. Again, we use the novel kinetic energy optimization criterion, adapted to calculate these several optimal parameters, and perform linear and nonlinear calculations with the optimal profiles. The results of the optimized VBM calculations are compared to the real-life measurements, the AB-simulations and simulations with MIKE 21 [11] and SWASH [12].

These two chapters comprise the contents of the technical report [8] and the published article [9]. The last chapter, however, has not been published yet.

We start Chapter 5 with the derivation of the reflection coefficient from the vertical step in the bottom profile, as well as the derivation of the classical WKB approximation. Then we calculate reflection coefficients of the VBM for the Booij's test case [1] and compare the obtained results to the results by Klopman \& Dingemans [7]. We also derive the analytical reflection-capable model based on WKB approximation and use it to obtain the reflection curve. VBM- and WKB-reflection results, being obtained with a simpler approach, are close to ones obtained by Klopman \& Dingemans. We also do additional simulations, varying a different parameter than in the previous works.

Finally, in the last Chapter 6 we briefly summarize the research done in this thesis, give a few recommendations and highlight possible directions for future advancements. 
[1] N. Booij, A note on the accuracy of the mild-slope equation, Coastal Engineering 7 (1983) 191-203.

[2] E. Bryant, Tsunami: The Underrated Hazard, (2001) Cambridge Univ. Press.

[3] K. Dysthe, H. Krogstad, P. Müller, Oceanic Rogue Waves, Ann. Rev. Fluid Mech. 40 (2008) 287-310.

[4] G. Gisler, Tsunami Simulations, Ann. Rev. Fluid Mech. 40 (2008) 71-90.

[5] E. van Groesen, Andonowati, L. She Liam, I. Lakhturov, Accurate modelling of uni-directional surface waves, J. Comput. Appl. Math. 234 (2010) 1747-1756.

[6] C. Kharif, E. Pelinovsky, Physical mechanisms of the rogue wave phenomenon, Eur. J. Mech. B, 22 (2003) 603-634.

[7] G. Klopman, M. W. Dingemans, Reflection in variational models for linear waves, Wave Motion 47 (2010) 469-489.

[8] I. Lakhturov, E. van Groesen, Optimized Variational Boussinesq Modelling; part 1: Broad-band waves over flat bottom, Internal report, University of Twente, The Netherlands. See http://eprints. eemcs . utwente.nl/.

[9] I. Lakhturov, D. Adytia, E. van Groesen, Optimized Variational ID Boussinesq Modelling for broad-band waves over flat bottom, Wave Motion 49 (2012) 309-322.

[10] MARIN, the Maritime Research Institute Netherlands, http:// www.marin.nl/web/Home. htm

[11] MIKE by DHI software, http://mikebydhi.com/

[12] SWASH, http://swash. sourceforge.net/ 

Generally speaking, wave motion is a phenomenon of energy transfer from one point in space to another without (or almost without) moving the mass of the continuum, whose oscillations are recognized as waves. There is a whole variety of oscillations and waves happening in different media and at all scales: from elementary particles, exhibiting wave-like properties, to gravitational waves in general relativity theory, piercing the whole universe. In the middle of this variety lie water waves, in particular, surface gravity waves studied in this thesis.

This chapter introduces the Variational Boussinesq Model (VBM), along with the $\mathrm{AB}$-equation, and presents some preliminaries needed to understand the material of the subsequent chapters. The VBM is derived in a manner of going from simpler to harder. Some details as regards numerical simulations are also provided.

\subsection{VARIATIONAL BOUSSINESQ MODEL}

In our research we restrict to waves over a layer of ideal fluid, i. e. fluid whose viscosity effects are neglected. Furthermore, we restrict to fluid which is incompressible, and to flow which is irrotational. We do not take into account such phenomena as surface tension, Coriolis forces or dissipation. The fluid is driven by influence of the gravitation, its flow satisfies mass and energy conservation laws [12].

Under these restrictions, at each moment of time the fluid motion must satisfy the Laplace equation in the interior of the fluid layer (see Figure 2):

$$
\triangle \Phi=0,
$$

along with surface and bottom conditions. Here $\Phi$ stands for the fluid velocity potential. It is a scalar function, depending on the spatial variables $x$ and $z$, and on the temporal variable $t$. Variable $x$ can be twodimensional, and then one speak about two horizontal dimensions, or three-dimensional flow. But in this thesis we restrict to the case when $x$ is a scalar, $\mathrm{i}$. e. the case of one horizontal dimension, or twodimensional flow.

It is important to note that the solution of the full nonlinear flow problem (1) can be complicated, resource-demanding and unnecessary. In many application areas one chooses to use Boussinesq models, which are models with reduced dimensionality, i. e. instead of solving the full 3- or 2-dimensional model, one derives some approximation of the initial model, but one dimension smaller, taking away a vertical 


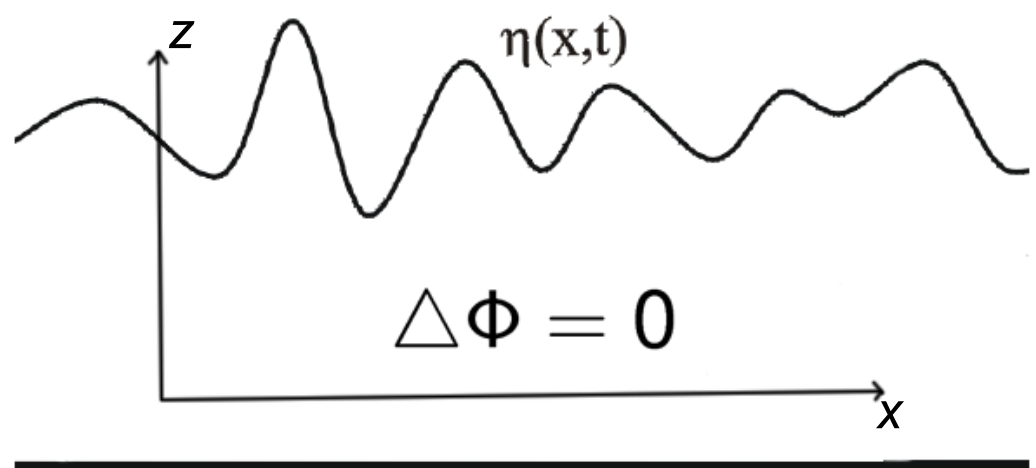

Figure 2: Fluid motion must satisfy the Laplace equation for $\Phi$ in the interior of the fluid layer. $\Phi$ is the fluid velocity potential, $\eta(x, t)$ is the wave elevation function.

variable, and solving then 2- or 1-dimensional model, correspondingly. Here we describe the VBM, a Boussinesq model, derived with the variational approach; in the following chapters we investigate its robustness and optimize its properties.

We start with the definition of the total energy, or Hamiltonian. It is the sum of the kinetic and potential energies:

$$
\mathcal{H}=\mathcal{K}+\mathcal{P}=\int H d x=\int K d x+\int P d x,
$$

where $H, K$ and $P$ are accordingly the total, kinetic and potential energy densities:

$$
\begin{gathered}
K=\frac{1}{2} \int_{-h}^{\eta}\left[\left(\partial_{x} \Phi\right)^{2}+\left(\partial_{z} \Phi\right)^{2}\right] d z, \\
P=g n \Rightarrow \mathcal{P}=\frac{1}{2} g \eta^{2} .
\end{gathered}
$$

In the following we use the canonical variables to describe the fluid equations, the surface elevation $\eta$ and the fluid potential $\phi$ at the free surface; $g$ is the gravitational acceleration. The surface fluid potential $\phi(x, t)=\left.\Phi(x, z, t)\right|_{z=\eta(x, t)}$ is the restriction of the (full) fluid potential $\Phi$ to the wave surface $\eta$. Luke's variational principle [11, 22] (see also $[2,25,30])$ reads as

$$
\delta \mathcal{L}=\delta \iint\left(\phi \partial_{t} \eta-K-P\right) d x d t=0 .
$$

Notice that variations of the Lagrangian $\mathcal{L}$ by $\phi$ and $\eta$, equated to zero,

$$
\delta_{\phi} \mathcal{L}=\iint\left(\partial_{t} \eta-\delta_{\phi} K\right) d x d t=0,
$$




$$
\delta_{\eta} \mathcal{L}=\iint\left(\partial_{t} \phi-\delta_{\eta} K-g \eta\right) d x d t=0,
$$

lead to the following Hamiltonian evolution equations:

$$
\left\{\begin{array}{l}
\partial_{t} \eta=\delta_{\phi} K \\
\partial_{t} \phi=-g \eta-\delta_{\eta} K .
\end{array}\right.
$$

This Hamiltonian description was first derived by Zakharov [30]. Exact equations are obtained if we could assure that the fluid potential $\Phi$ satisfies the Laplace equation in the interior, the impermeability condition at the bottom $z=-h$ and has the prescribed value $\Phi=\phi$ at the free surface $z=\eta$. In this chapter and further we consider bottom bathymetry to be flat; the case of varying bottom is dealt with in Chapter 5 .

The exact potential $\Phi$ has the extremal property — it minimizes the kinetic energy over all potentials that satisfy the prescribed surface value $\phi$. This minimization property of the kinetic energy will be exploited later in the thesis to obtain the best dispersive properties of the VBM.

Since the Laplace problem cannot be solved explicitly for nontrivial $\eta$, the kinetic energy has to be approximated to make the model useful for numerical simulations. Every feasible kinetic energy approximation in the VBM will be obtained by approximating the velocity potential $\Phi$. Depending on approximation, system (6) will be expanded with elliptic equations, to be solved together with evolution equations.

\subsubsection{Linear Shallow Water Equation}

At first, we would like to give a rather trivial example. It can be shown that the Linear Shallow Water Equation (LSWE) can be seen as a particular case of the VBM. Indeed, take $\Phi$ independent of the vertical variable:

$$
\Phi(x, z, t) \approx \phi(x, t), \forall z \in[-h, \eta(x, t)] .
$$

This means that the fluid particles do not move in the vertical direction.

The kinetic energy (3) transforms then to

$$
K=\frac{1}{2}(\eta+h)\left(\partial_{x} \phi\right)^{2},
$$

and for small-amplitude waves can be approximated as

$$
K \approx \frac{h}{2}\left(\partial_{x} \phi\right)^{2}
$$

Thus, after taking variations, the evolution equations for the constant depth $h_{0}$ become

$$
\left\{\begin{array}{l}
\partial_{t} \eta=-h_{0} \partial_{x}^{2} \phi \\
\partial_{t} \phi=-g \eta
\end{array}\right.
$$


Notice, this immediately leads to the well-known LSWE or the wave translation equation:

$$
\partial_{t}^{2} \eta=c_{0}^{2} \partial_{x}^{2} \eta
$$

where $c_{0}=\sqrt{g h_{0}}$ is the shallow water phase speed.

A variation of the LSWE, valid for the varying bottom, will be derived and used in Chapter 5 in calculations of the reflection from the bathymetry features.

\subsubsection{VBM with one profile}

Of course, the LSWE covers a very limited range of applications. In oceanography it was mostly (and quite extensively) used in tsunami simulations. To make VBM more useful one needs to improve the kinetic energy approximation. This is usually done for the VBM by approximating the fluid potential function $\Phi$ (see e.g. [17]). In this subsection we present a VBM variant, which we call further the VBM with one profile; the word 'profile' is related to a (vertical dispersion) function $F(z)$, which greatly affects dispersive properties of the model.

Represent $\Phi$ in the form:

$$
\Phi(x, z, t) \approx \phi(x, t)+F(z) \psi(x, t),
$$

where $\psi$ is an unknown function, but the vertical profile $F$ will be chosen in advance (this is usually done with help of a parabolic function [18], but futher we will show that the cosh-profile is much better). The bottom impermeability condition $\left.\frac{\partial \Phi}{\partial n}\right|_{z=-h(x)}=0$ where $n$ is a normal to the bottom, for the case of flat bottom implies $\partial_{z} \Phi(-h)=0$ or $F^{\prime}(-h)=0$. Also the condition that on the surface $F(\eta)=0$ should be satisfied. Taking the assumption that the amplitude of waves is small, this immediately transforms into $F(0)=0$.

The kinetic energy density (3) expands to

$$
\begin{gathered}
K=\frac{1}{2} \int_{-h}^{\eta}\left[\left(\partial_{x} \phi+F \partial_{x} \psi\right)^{2}+\left(F^{\prime} \psi\right)^{2}\right] d z \\
=\frac{1}{2}(\eta+h)\left(\partial_{x} \phi\right)^{2}+\partial_{x} \psi \partial_{x} \phi \int_{-h}^{\eta} F d z+\frac{1}{2}\left(\partial_{x} \psi\right)^{2} \int_{-h}^{\eta} F^{2} d z+\frac{1}{2} \psi^{2} \int_{-h}^{\eta}\left(F^{\prime}\right)^{2} d z
\end{gathered}
$$

and for waves of small amplitude can be approximated as

$$
K \approx \frac{1}{2}\left[h\left(\partial_{x} \phi\right)^{2}+\alpha\left(\partial_{x} \psi\right)^{2}+\gamma \psi^{2}+2 \beta \partial_{x} \phi \partial_{x} \psi\right],
$$

where coefficients $\alpha, \beta$ and $\gamma$ are constants (in case of the parabolic approximation) or depend on parameters (the $\kappa$-parameter in the next chapter):

$$
\alpha=\int_{-h}^{0} F^{2} d z, \quad \beta=\int_{-h}^{0} F d z, \quad \gamma=\int_{-h}^{0}\left(F^{\prime}\right)^{2} d z
$$


Their exact values are given in Chapter 3 by formulae (52) and (68).

The Lagrangian is then given by

$$
\begin{gathered}
\mathcal{L}(\eta, \phi, \psi)=\int_{0}^{T} \int_{0}^{L}\left[\phi \partial_{t} \eta-\frac{1}{2} g \eta^{2}\right. \\
\left.-\frac{1}{2}\left\{h\left(\partial_{x} \phi\right)^{2}+\alpha\left(\partial_{x} \psi\right)^{2}+\gamma \psi^{2}+2 \beta \partial_{x} \phi \partial_{x} \psi\right\}\right] d x d t .
\end{gathered}
$$

Notice, in addition to solving the evolution equations (6) we ought to solve an additional 1-dimensional elliptic equation in the interior of $x$-domain. Indeed, the Euler-Lagrange equations of the evolution system (6) are extended to:

$$
\left\{\begin{array}{l}
\partial_{t} \eta=\delta_{\phi} K \\
\partial_{t} \phi=-g \eta-\delta_{\eta} K \\
\delta_{\psi} K=0
\end{array}\right.
$$

The equation $\delta_{\psi} K=0$ after partial integrations becomes

$$
-\alpha \partial_{x}^{2} \psi+\gamma \psi=\beta \partial_{x}^{2} \phi
$$

Hence, the dynamic PDE system of the linear VBM with one profile looks like:

$$
\left\{\begin{array}{l}
\partial_{t} \eta=-h \partial_{x}^{2} \phi-\beta \partial_{x}^{2} \psi \\
\partial_{t} \phi=-g \eta \\
-\alpha \partial_{x}^{2} \psi+\gamma \psi=\beta \partial_{x}^{2} \phi,
\end{array}\right.
$$

where coefficients $\alpha, \beta$ and $\gamma$ can be pre-calculated before solving the PDE system. For this, of course, the vertical profile function $F$ should be chosen in advance. This choice largely defines dispersive properties of the model (see Subsection 2.1.5 further).

The VBM with one profile is extensively used in Chapter 3, where derivation of the model and its dispersion relation will be given with more details. The adapted version of the model, which can simulate waves over bathymetry, is investigated in Chapter 5 , in particularly, to calculate reflection coefficients of the model.

Going through the same derivations, but taking into the account bathymetry $h(x)$, with so called mild-slope approximation (i. e. when the $x$-derivative of the function $F$ is negligible) and without linearization, one would obtain the nonlinear VBM system with one profile:

$$
\left\{\begin{array}{l}
\partial_{t} \eta=-\partial_{x}\left((h+\eta) \partial_{x} \phi\right)-\partial_{x}\left(\beta \partial_{x} \psi\right) \\
\partial_{t} \phi=-g \eta-\frac{1}{2} \partial_{x} \phi^{2} \\
-\partial_{x}\left(\alpha \partial_{x} \psi\right)+\gamma \psi=\partial_{x}\left(\beta \partial_{x} \phi\right) .
\end{array}\right.
$$

In this case, together with the depth function $h(x)$, the integral coefficients $\alpha, \beta$ and $\gamma$ will depend on the spatial coordinate $x$. Moreover, with allowing nonlinearity, they will be represented by integrals not up to the still water level, but to the wave surface $\eta(x, t)$, so they have to be recalculated on each time step for each point of a domain. 


\subsubsection{VBM with multiple profiles}

Now let us take the more complicated form of the $\Phi$-Ansatz:

$$
\Phi(x, z)=\phi(x)+\Sigma_{m} F_{m}(z) \psi_{m}(x)=\phi(x)+F(z) \cdot \Psi(x)
$$

with the appropriate bottom and surface conditions on functions $F_{m}$. Here notations of vector-functions is used for $F$ and $\Psi$.

The kinetic energy (3) becomes in this case:

$$
K \approx \frac{1}{2} \int\left[(h+\eta)\left(\partial_{x} \phi\right)^{2}+\alpha \partial_{x} \Psi \cdot \partial_{x} \Psi+\gamma \Psi \cdot \Psi+2 \partial_{x} \phi \beta \cdot \partial_{x} \Psi\right] d x,
$$

where instead of scalar coefficients, as in the previous subsection, we have now integral coefficients that are elements of the matrices $\alpha$ and $\gamma$ and the column vector $\beta$ :

$$
\alpha_{j m}=\int_{-h}^{\eta} f_{j} f_{m} d z, \quad \beta_{m}=\int_{-h}^{\eta} f_{m} d z, \quad \gamma_{j m}=\int_{-h}^{\eta} f_{j}^{\prime} f_{m}^{\prime} d z .
$$

And again, they depend on the approximation of the vertical potential profiles $F$. These profiles are the cosh-profiles, given by (122) where $\kappa_{m}$ is a wave number to be chosen optimally in the next chapters.

Taking variations of the Lagrangian, as in the previous subsection, we arrive at the ultimate PDE-system :

$$
\left\{\begin{array}{l}
\partial_{t} \eta=-\partial_{x}\left((h+\eta) \partial_{x} \phi\right)-\partial_{x}\left(\beta \partial_{x} \Psi\right) \\
\partial_{t} \phi=-g \eta-\frac{1}{2} \partial_{x} \phi^{2} \\
-\partial_{x}\left(\alpha \partial_{x} \Psi\right)+\gamma \Psi=\partial_{x}\left(\beta \partial_{x} \phi\right)
\end{array}\right.
$$

Here the last equation is in fact an elliptic system of PDEs. The matrices $\alpha$ and $\gamma$ are symmetric, and the vector $\beta$ consists of the corresponding coefficients $\beta_{i}$. The vector $\Psi$ consists of the functions $\psi_{k}(x, t)$ to be found at each time step. There are as many interconnected elliptic equations, as there are functions $\psi_{k}$ in the Ansatz (17).

This form of the VBM is used throughout Chapter 4, in order to obtain the best dispersive characteristics, unachievable with any choice of the F-function in the case of the one-profile VBM. .

\subsubsection{Numerical implementation}

The VBM is obtained from the variational formulation by minimizing the Lagrangian (12) with respect to $\eta, \phi$ and $\psi_{m}$, so, it is natural to build an implementation with the Finite Element Method (FEM). One can use piecewise linear local basis functions $T_{k}(x)$ since the highest derivatives in (12) are of the first order. We discretize the solutions in 
space by FEM; this leads to a system of ordinary differential equations (ODEs), which we solve in time by a Runge-Kutta method. It is most convenient to derive the numerical system from the weak formulation.

For the simplicity of the example, let us define the numerical implementation of the one-dimensional linear VBM with one profile over varying bathymetry (16).

First of all, we consider the spatial domain as the segment $[0, L]$, and take the spatial grid $x_{i}$ on it, where $x_{1}=0$ and $x_{n}=L$. The grid can be equidistant or not (for example, changed according to depth variations). We define the vector of $\eta$-values in the grid points:

$$
\eta_{i}(t)=\eta\left(x_{i}, t\right)
$$

Similar arrangement we have for functions $\phi, \psi, h$, and coefficients $\alpha, \beta$ and $\gamma$. On the same grid points we define the piecewise-linear tent functions $T_{i}(x)$ with value one in the point $x_{i}$ and zero in the neighbouring grid points. By definition, the distance between two grid points, $\triangle x_{i}=x_{i+1}-x_{i}$, is the length of the element $e_{i}=\left[x_{i}, x_{i+1}\right]$.

We approximate $\eta, \phi$ and $\psi$ as superposition of tent functions:

$$
\begin{aligned}
& \eta(x, t)=\sum_{i} \eta_{i}(t) T_{i}(x) \\
& \phi(x, t)=\sum_{i} \phi_{i}(t) T_{i}(x) \\
& \psi(x, t)=\sum_{i} \psi_{i}(t) T_{i}(x) .
\end{aligned}
$$

Taking variations of the Lagrangian (12) in (5) by $\phi, \eta$ and $\psi$ in the directions $\delta \phi, \delta \eta$ and $\delta \psi$ appropriately, substituting the test functions $\delta \phi, \delta \eta$ and $\delta \psi$ with the element functions $T_{i}$, and gathering them into matrices, we arrive at the matrix equations:

$$
\left\{\begin{array}{l}
M \partial_{t} \underline{\eta}=D^{h} \underline{\phi}+D^{\beta} \underline{\psi} \\
M \partial_{t} \underline{\phi}=-g M \underline{\eta} \\
-D^{\alpha} \underline{\psi}-M^{\gamma} \underline{\psi}=D^{\beta} \underline{\phi} .
\end{array}\right.
$$

One can find values of the matrices $M$ and $D$ in Section 7.2. The discrete system (21) is nothing else but a high-dimensional system of ordinary differential equations, which can be solved by the Runge-Kutta method.

\section{Algorithm for evolution simulation}

1. Build up the grid $x_{i}$. An equidistant grid can be used. Another possibility is to vary a grid cell size according to depth (over smaller depth a wave length is shrinking, so the grid there should become finer).

2. Having a bathymetry $h_{i}$ on the grid $x_{i}$, pre-calculate $\kappa_{i}=\kappa\left(x_{i}\right)$ according to the kinetic-energy minimization principle (see Subsections 3.3.2 and 4.3.1 in the following chapters). 
3. Pre-calculate coefficients $\alpha_{i}, \beta_{i}$ and $\gamma_{i}$ according to cosh-profile (see Section 7.I for exact coefficients values).

4. Build up matrices $M, D, M^{\gamma}, D^{\alpha}, D^{\beta}$ and $D^{h}$ (see Section 7.2 for exact element values).

5. Start a Runge-Kutta ODE-solver. We normally use ode45 function in MATLAB.

6. For every fixed time $t$ we have the vectors $\eta$ and $\phi$, obtained from the previous step of the ODE solver.

7. Given this $\phi$ and using the third equation of (16), we calculate a new vector $\underline{\psi}^{+}$.

8. Using the first two equations from the system (16) and a vector $\psi^{+}$, we calculate new vectors $\eta^{+}$and $\phi^{+}$, according to the RungeKutta algorithm.

9. Repeat from step 6.

The situation becomes slightly more complicated in case of the VBM with multiple profiles. The cost of the calculation on Step 7 rises, as one must find several vectors $\psi$ instead of one. One can find the corresponding numerical system (72) in Subsection 4.2.3.

\subsubsection{Dispersion of the model}

The choice of the vertical profile (or profiles) $F$ determines dispersive properties of the model. It was usual for the VBM to choose a parabolic profile that satisfies bottom and surface conditions (see e. g. [14, 15]). The parabolic VBM is discussed in Chapter 3. It should be remarked that the parabolic VBM would satisfy needs of tsunami simulations up to the coastal zone, as rather long waves have the appropriate propagation speed.

However, when it comes to the laboratory waves with broad spectra, waves over a deep sea or e. g. a monochromatic wave train of a specific (high) frequency, the cosh VBM with one vertical profile demonstrates its superiority over the previous choice, this is shown in Chapter 3 (or corresponding technical report [21]). The dispersion accuracy for the long waves stays approximately the same as in the parabolic VBM. But additionally, for one specific wave number $\kappa$ the model obtains an exact dispersion as derived in the Airy wave theory, including the phase speed and the group speed.

For applications, where the wave spectrum is very broad, even performance of the optimized cosh VBM with one vertical profile can become incomplete. This is not a rare case: for example, for waves having the realistic JONSWAP spectrum the wave length in the spectral tail 
can become several times smaller than in the spectral peak. As demonstrated in Subsections 3.5.2 and 3.5.3, such short waves can become important for the appropriate focussing of a wave train, adding up to $15-20 \%$ of the wave amplitude.

For such extreme test cases the optimized cosh VBM with multiple vertical profiles, presented with more details in Chapter 4 (or corresponding paper [20]), shows really good performance. Already with two vertical profiles the dispersion accuracy errors become small enough to allow for almost exact reproduction of the real-life wave shape and amplitude. This can be done partially thanks to the appropriate choice of the parameters. For the effective wave numbers $\kappa_{j}$-s, used in this type of VBM, Airy theory provides the exact dispersion. It will be shown how to find these wave numbers $\kappa_{j}$-s in an optimal way, using the novel kinetic energy criterion, minimizing the kinetic energy error and simultaneously minimizing the error in phase and group speed.

The material of the subsequent chapters relies a lot on the analytic expressions of the dispersion, which will be obtained from the derived PDE systems of the VBM. The derivation and formulae will be given in Subsections 3.2.1 and 4.2.2, followed by plots of both phase and group speeds and further analysis.

Here we only make the remark that one can obtain expressions not only for the VBM's dispersion, but it is possible also to obtain analytically the dispersion of the numerical scheme. We do not provide formulae of the numerical dispersion here (for they are large), but show a plot of the phase and group speed of the parabolic VBM (both model's and numerical dispersion), along with the exact dispersion; see Figure 3 .

It is visible that the model's phase speed is much larger than the exact phase speed, having non-zero limit for short waves. This causes the waves in the model to propagate faster than real waves. Although the situation is much better in case of the cosh VBM, it can be shown, that the non-zero limit is always the case (at least for the VBM with one profile, see expression (49) in Subsection 3.2.1).

Moreover, as regards the phase speed in the numerical scheme, it is even larger than the model's dispersion. Only until a certain boundary they approximately coincide. Of course, by taking finer grid the range of simulated wave lengths will grow, and appropriately this boundary will shift further into the region of short waves. The curve of the numeric group speed grows only until certain point and then falls rapidly to zero. Still, it remains approximately the same as the model's group speed until the same point.

This implies importance of choosing a sufficiently fine grid. On the other hand, whichever discretization we take, we can come up only with the model's phase speed at best. When for the range of simulated 

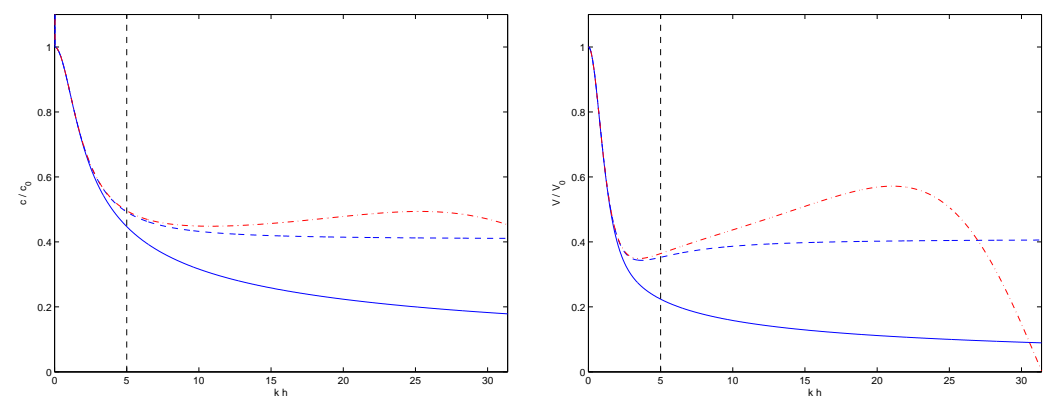

Figure 3: At the left the normalized phase speed and at the right the normalized group speed are shown for the exact dispersion (solid), the parabolic VBM with one profile (dashed) and for the FEM numerical scheme (dash-dot). On horizontal scale $k h$ is a wave number multiplied by depth. For the numeric dispersion the whole spectrum of waves simulated by the numerical scheme is presented (that is, the distance between grid points is $\triangle x=0.1 \mathrm{~m}$ ). The vertical bar (dashed) represents the boundary, until which the numerical dispersion approximately coincides with the dispersion of the model.

wave numbers model's dispersion differ much from the exact (or real) dispersion, any numerical tricks will be in vain. That is, to improve the results of simulations, which depend much on the dispersive properties, first of all, we have to improve the model's dispersion. In the following chapters 3 and 4 we show how to approach this task efficiently.

\subsubsection{Advantages of VBM}

We would like to defer the demonstration of the VBM capabilities as regards dispersion improvements to the next chapters. Here we would like to stress important properties of Boussinesq models and, in particular, advantages of the VBM. These model's tratis, in our opinion, should lead to the successful use of the VBM in practical applications.

Dimensions reduction is a common feature of all Boussinesq-like models. Instead of solving the full nonlinear flow in 3 or 2 dimensions, one derives some approximation of the initial model, but one dimension smaller, taking away the vertical variable and solving then 2- or 1-dimensional model, correspondingly. There is no reason to solve the complicated and resource-demanding full flow problem, when only the wave elevation function is required (or e. g. some depth-averaged characteristics of the solution).

At the beginning of their development, Boussinesq-like models were essentially shallow-water models, i. e. valid for a confined range of long waves, and with limited nonlinearity. During recent decades the 
two major tendencies were driving improvements in this field: struggle for better dispersion and for better nonlinearity [13]. The accurate dispersion is believed to be an important requirement for the improvement of various properties of a Boussinesq model $[23,24]$. In this thesis we concentrate mostly on the dispersive characteristics, and there will be a lot said about them in the following chapters. It will be demonstrated that the VBM can achieve excellent linear dispersion (the nonlinear properties of the VBM are not studied thoroughly in this thesis, but one can find nonlinear simulations in Chapter 4). Even more important to note, this gain is achieved without sacrificing any other beneficial properties of the VBM, specific to the discussed model.

The VBM is derived with the variational approach, and possesses positive-definite Hamiltonian. Thanks to this, the variational structure of the model is transferred to the FEM discretization, and the used numerical implementation conserves mass and energy (apart from minor dissipation inherent in a Runge-Kutta ODE solver). This is in contrast to some Boussinesq-like models, where energy can become negative for very short waves, which, in turn, can lead to instabilities of the numerical code [18]. There is also momentum conservation in the VBM, provided that the bottom profile is horizontal.

Many models contain higher-order derivatives and mixed spacetime derivatives, causing inconvenience in practical applications. The PDE-system of the VBM, in contrast, contains at most second order derivatives; see e. g. (16). In the weak formulation this becomes at most order one, and the simplest element functions can be used in the FEM discretization of the model, as explained in Subsection 2.2.2. The lack of mixed space-time derivatives allows separating spatial and temporal discretizations in the numerical code. For instance, we prefer to use FEM for spatial, and Runge-Kutta for temporal solution of the dynamic equations.

Other useful advantages of the VBM include the simplicity of the model's generalization to two horizontal dimensions (see e. g. [5]), generalization to varying bathymetry (see e. g. [6]) and good shoaling characteristics $[5,16]$. In particular, we show in Chapter 5 that reflection coefficients are close to the exact ones (see also $[16,18]$ ). This allows to use the VBM in a wide range of coastal applications.

\subsection{UNI-DIRECTIONAL AB-EQUATION}

This section concerns the recently derived so-called AB-equation [9], which models waves varying in time and in only one horizontal direction (unlike the VBM, which is a bi-directional model). Here we briefly demonstrate how to derive the uni-directional AB-equation, describe shortly its pseudo-spectral numerical implementation and use it to simulate the Highest Stokes Wave. 
This section is mainly an excerpt from the published article [9]. In the following Chapters 3 and 4 the described AB-equation serves as the main vehicle for purposes of comparison of the VBM to the auxiliary model.

\subsubsection{Derivation of $A B$-equation}

We consider both cases of a horizontal bottom and an infinitely deep layer of water. We start to introduce the uni-directionalization method for the linear equations, after which we will derive the approximate $\mathrm{AB}$-equation for nonlinear equations.

As it will be demonstrated shortly, the AB-equation is capable of incorporating any dispersion relation. We normally use the exact dispersion relation for infinitesimal waves, as derived in linear theory:

$$
\omega^{2}=g k \tanh (k h)
$$

with $k$ the wave number and $h$ the depth. We introduce a pseudodifferential (linear dispersive) operator $A$ which is defined by its symbol:

$$
\hat{A}(k)=i k \sqrt{\tanh (k h) / k} ;
$$

note that defined in this way, $A$ is a skew-symmetric, real operator. Then the kinetic energy is

$$
K_{\text {lin }}=\frac{1}{2} \int(A \phi)^{2} d x
$$

and the linear equations are

$$
\partial_{t} \eta=-A^{2} \phi, \quad \partial_{t} \phi=-g \eta,
$$

equivalent with the second order equation

$$
\partial_{t}^{2} \eta=g A^{2} \phi
$$

Exact uni-directionalization for linear equations. In the simplest model, non-dispersive and linear, the equation for the surface elevation reads $\left(\partial_{t}^{2}-c^{2} \partial_{x}^{2}\right) \eta=0$, with general solution $\eta(x, t)=f(x-c t)+$ $g(x+c t)$. Here, $f$ and $g$ are arbitrary profile functions, and $f(x-c t)$ denotes the wave running undisturbed in shape to the right and similarly $g(x+c t)$ running to the left. An initial hump released without initial velocity will split precisely in two: one to the right, the other to the left, each with half of the initial elevation height. If the hump is confined in space, after some time the two parts are separated, and it is tempting to look for the equation that describes only the right travelling part. For the simple equation above, this is easy. It is the first order uni-directional equation given by $\left(\partial_{t}+c \partial_{x}\right) \eta=0$. We observe 
that the possibility to split the two waves is based on the fact that $\partial_{t}^{2}-c^{2} \partial_{x}^{2}=\left(\partial_{t}+c \partial_{x}\right)\left(\partial_{t}-c \partial_{x}\right)=\left(\partial_{t}-c \partial_{x}\right)\left(\partial_{t}+c \partial_{x}\right)$. Of course, this is only true provided $c$ is constant, i.e. for horizontal bottom. A non-flat bottom leads to $c$ depending on $x$, and waves cannot be split exactly: a wave initially travelling to the right will be partly reflected, leading to a right travelling wave, etc.; then uni-directionalization can at best be approximate. The same applies when the equation is nonlinear.

As a direct generalization of the above case, uni-directionalization is possible for any linear dispersive wave equation (with constant coefficients). For a dispersion operator $L$, the equation $\left(\partial_{t}^{2}-L^{2}\right) \eta=0$ can be written as $\left(\partial_{t}+L\right)\left(\partial_{t}-L\right) \eta=0$, and the uni-directional equations are $\left(\partial_{t} \pm L\right) \eta=0$.

We will use this generalization in the following for $L=\sqrt{g} A$ with $A$ as above for infinitesimal waves. We observe that since the original equations are given by $\partial_{t} \eta=-A^{2} \phi_{0}$, and $\partial_{t} \phi_{0}=-g \eta$, for unidirectional waves satisfying $\left(\partial_{t}+\sqrt{g} A\right) \eta=0$ the potential and elevation are related by

$$
\phi_{0}=\sqrt{g} A^{-1} \eta
$$

We here introduced the subscript for $\phi_{0}$ to remember that this is the potential at the flat surface according to linear theory.

Uni-directionalization by restriction of the action principle. It is useful to derive the dynamic equation in a different way using the canonical action principle (5) for the linear problem:

$$
\text { Crit }_{\phi, \eta} \int d t\left\{\int \phi_{0} \partial_{t} \eta d x-\mathcal{H}_{\text {lin }}\left(\phi_{0}, \eta\right)\right\},
$$

where $\mathcal{H}_{\text {lin }}\left(\phi_{0}, \eta\right)=\frac{1}{2} \int\left(A \phi_{0}\right)^{2} d x+\int \frac{1}{2} g \eta^{2} d x$. By restriction to the set $\phi_{0}=\sqrt{g} A^{-1} \eta$ one gets

$$
\text { Crit }_{\eta} \int d t\left\{\int \sqrt{g} A^{-1} \eta \partial_{t} \eta d x-\mathcal{H}_{u n i}(\eta)\right\}
$$

with

$$
\mathcal{H}_{u n i}(\eta)=\int g \eta^{2} d x
$$

This restricted canonical action principle leads to the correct equation for $\eta$ as found above: $\left(\partial_{t}+\sqrt{g} A\right) \eta=0$. Note that the Hamiltonian $\mathcal{H}_{\text {uni }}$, being the sum of potential and kinetic energy, is precisely twice the potential energy, expressing equipartition of energy as is known for linear equations.

Derivation of the AB-equation. Following the derivation in Van Groesen \& Andonowati [9], we use the above method to incorporate nonlinear effects. The basic idea is to use the fluid potential at the free 
surface $\phi_{0}$ as auxiliary variable and approximate the potential above the still water level with a few Taylor terms. To that end we introduce the vertical velocity at the still water level $W_{0}:=\partial_{z} \Phi(x, z=0, t)$. Then we have for instance $\Phi(x, z, t)=\phi_{0}+z W_{0}+O\left(z^{2}\right)$. According to linear theory it holds that $W_{0}=-A^{2} \phi_{0}$.

We now consider the approximation for the kinetic energy. This is done by splitting the kinetic energy in a term up to the still water level and a term accounting for the actual surface elevation as

$$
K=\iint^{z=0} \frac{1}{2}|\nabla \Phi|^{2} d z d x+\iint_{z=0}^{z=\eta} \frac{1}{2}|\nabla \Phi|^{2} d z d x
$$

The first term is the term of linear theory, $\iint^{z=0} \frac{1}{2}|\nabla \Phi|^{2} d z d x=\int \frac{1}{2}\left(A \phi_{0}\right)^{2} d x$. Imposing the uni-directionalization restriction (25), we find the quadratic expression $\mathcal{H}_{\text {uni }}$ of linear theory as could be expected.

For the other term we take the approximation

$$
\iint_{z=0}^{z=\eta} \frac{1}{2}|\nabla \Phi|^{2} d z d x=\frac{1}{2} \int \eta\left[\left(\partial_{x} \phi_{0}\right)^{2}+W_{0}^{2}+O\left(\eta^{3}\right)\right] d x .
$$

This approximation is motivated by the Taylor expansion of the potential near the still water level, together with the uni-directionalization restriction which relates $\phi_{0}$ to the surface elevation. This explains the order term, where, here and in the following, we will use the order symbol to denote the lowest degree of the polynomial in the surface height and all its derivatives. Imposing the uni-directionalization restriction, one gets the cubic terms in an explicit way; the restricted Hamiltonian which is correct up to and including the third order is then found to be

$$
\bar{H}(\eta)=g \int\left[\eta^{2}+\frac{1}{2} \eta\left\{(A \eta)^{2}+(B \eta)^{2}\right\}\right] d x
$$

Here we have introduced for notational simplicity the symmetric operator $B=\partial_{x} A^{-1}$. Notice, given the dispersion operator $C$, the operators $A$ and $B$ will be expressed via it as

$$
A=C \partial_{x} / \sqrt{g}, \quad B=\sqrt{g} C^{-1} .
$$

To implement the same restriction in the action, we use $\phi=\phi_{0}+$ $\eta W_{0}+O\left(\eta^{3}\right)$, which results into

$$
\iint \phi \partial_{t} \eta d x d t=\sqrt{g} \iint\left[A^{-1} \eta-\eta A \eta\right] \partial_{t} \eta d x d t
$$

Although correct, the expression $\eta A \eta \partial_{t} \eta$ will lead to rather complicated expressions. We will simplify this term by taking the lowest order approximation for $\partial_{t} \eta=-\sqrt{g} A \eta+O\left(\eta^{2}\right)$, so that

$$
\iint \sqrt{g}\left[A^{-1} \eta-\eta A \eta\right] \partial_{t} \eta d x d t=\iint\left[\sqrt{g} A^{-1} \eta \partial_{t} \eta+g \eta(A \eta)^{2}\right] d x d t .
$$


Then we can rewrite the action principle as

$$
\mathcal{A}_{A B}(\eta)=\int\left[\int \sqrt{g} A^{-1} \eta \partial_{t} \eta d x-H_{A B}(\eta)\right] d t,
$$

where the modified Hamiltonian $H_{A B}$ contains a term from the original action and is given by

$$
H_{A B}(\eta)=g \int\left[\eta^{2}+\frac{1}{2} \eta\left\{(B \eta)^{2}-(A \eta)^{2}\right\}\right] d x
$$

The resulting equation $\delta \mathcal{A}_{A B}(\eta)=0$ reads

$$
\partial_{t} \eta=-\frac{A}{2 \sqrt{g}} \delta H_{A B}(\eta)
$$

This equation was called the $A B$-equation in [9] because of the distinct role of the operators $A$ and $B$ that appear in the full expression of the equation:

$$
\partial_{t} \eta=-\sqrt{g} A\left[\eta+\frac{1}{4}(B \eta)^{2}+\frac{1}{2} B(\eta B \eta)-\frac{1}{4}(A \eta)^{2}+\frac{1}{2} A(\eta A \eta)\right] .
$$

The dispersive properties are clearly present in the four quadratic terms through the operators $A$ and $B$. This dispersion applies for all depths, including the limit for infinite depth. For infinite depth, the operators are given by their limiting expressions by

$$
\hat{A}(k)=i \operatorname{sign}(k) \sqrt{|k|}, \quad \hat{B}(k)=\sqrt{|k|} .
$$

From this it becomes clear that all 4 terms in the AB-equation are of the same dispersive order. On the other hand, for shallower water, $\hat{A}(k) \approx i k \sqrt{h}, \hat{B}(k) \approx 1 / \sqrt{h}$, and the 'A-terms' are negligible compared to the 'B-terms':

$$
\partial_{t} \eta \approx-\sqrt{g} A\left[\eta+\frac{3}{4 h} \eta^{2}\right]
$$

This result is close to the classical KdV-equation, see below.

In all the preceding steps, the total action functional has been approximated correctly up to and including cubic terms in the wave height. As a consequence, the AB-equation is second order accurate in the wave height and applicable both for finite depth dispersion and for infinite depth dispersion.

The AB-equation is a generalised Hamiltonian dynamical equation, since the cosymplectic operator $A$ is skew-symmetric. As an immediate consequence, the total energy is conserved for solutions

$$
\partial_{t} H_{A B}(\eta)=0
$$


Moreover, since $A$ is space independent, the equation is translationinvariant. This continuous symmetry corresponds to another conserved functional, the momentum functional

$$
M=g \int \eta \cdot B \eta d x
$$

This functional is the restriction of the corresponding momentum functional of the exact equations, which is $\int \eta \cdot \partial_{x} \phi d x$. With this momentum functional, the AB-equation can also be written in the way of Benjamin [1] as $\partial_{t} \delta M(\eta)=-\partial_{x} \delta H(\eta)$; hence solutions have a constant center of mass velocity.

Approximations. Within the restriction to second order accuracy in the wave height, the AB-equation is valid for waves of all wavelength since the operators $A, B$ lead to exact dispersion relation for small waves. There are many other uni-directional wave equations that are derived under specific restrictions for the wavelength / depth ratio; this is the reason that such approximations have only been derived for finite depths. For finite depth, the non-rational pseudo-differential operators $A, B$ can be expanded and truncated to a certain order. In that way, many approximations can be derived from the AB-equation in a consistent way. One of these is the most famous uni-directional equation, the one named after Korteweg \& de Vries, the classical KdVequation. The equation and its standard Hamiltonian structure are given by

$$
\begin{aligned}
\partial_{t} \eta & =-c_{0} \partial_{x}\left[\left(1+\frac{h^{2}}{6} \partial_{x}^{2}\right) \eta+\frac{3}{4 h} \eta^{2}\right] \\
& =-c_{0} \partial_{x} \delta H_{K d V} \text { for } H_{K d V}=\int\left(\frac{1}{2} \eta^{2}-\frac{h^{2}}{12}\left(\partial_{x} \eta\right)^{2}+\frac{1}{4 h} \eta^{3}\right) d x
\end{aligned}
$$

This coincides with the result (3I) by taking the approximation $\hat{A}(k) \approx$ $i k \sqrt{h}$ acting on the nonlinear terms, and a next order expansion $\hat{A}(k) \approx$ $i k \sqrt{h}\left(1-(h k)^{2}\right) / 6$ acting on the linear term. Although such 'uncontrolled' approximations may easily destroy the variational structure, in this case the final result, the KdV-equation, is a Hamiltonian system again. See [9] for further examples of consistent approximations that may be useful in cases of less stringent conditions on wavelength or amplitude.

\subsubsection{Numerical implementation}

Here we describe a simple algorithm of the AB-equation simulation, which uses pseudo-spectral technique. We assume that the Initial Value Problem has to be solved. For details about the signalling problem, see Section 2.3. 
The main calculation is done in Fourier space, to which we transfer the equation (30):

$$
\left.\partial_{t} \hat{\eta}=-\sqrt{g} \hat{A} \cdot\left[\hat{\eta}+\frac{1}{4} \widehat{(B \eta)^{2}}+\frac{1}{2} \widehat{B(\eta B \eta}\right)-\frac{1}{4} \widehat{(A \eta)^{2}}+\frac{1}{2} \widehat{A(\eta A \eta)}\right],
$$

with the hat notation for the spatial Fourier transform. In the Fourier space operator applications are just inner-product multiplications. However, from the numerical point of view, some terms here should be calculated in the real space. For instance, for the nonlinear terms like $\eta \cdot B \eta$, it is better to calculate the operator's application $B \eta$ in the Fourier space, and then to convert the vector back to the real space to perform the multiplication $\eta \cdot B \eta$. Otherwise, in the Fourier space it would be a costly convolution operation.

\section{Algorithm for evolution simulation}

1. Build up the equidistant grid $x_{i}$. Discretize the given initial function $\eta(x, t=0)$ to obtain the vector $\underline{\eta_{0}}$.

2. Transfer this $\eta_{0}$ vector to the Fourier space and obtain $\hat{\eta}_{0}$. The size of the grid is equal to the number of Fourier modes in the calculation.

3. Given the (approximate) dispersion operator $C$ and its symbol $\hat{C}(k)$, where $k$ is the wave number, calculate symbols of the operators $A$ and $B$ according to formulae (26). We usually use the exact (linear) dispersion (22).

4. Start a Runge-Kutta ODE-solver. We normally use ode45 function in MATLAB.

5. For every fixed time $t$ we have the vector $\hat{\eta}$, the Fourier transform of the wave elevation function, which is obtained from the previous step of the ODE solver.

6. Apply operators in Fourier space: $\hat{A} \cdot \hat{\eta}$ and $\hat{B} \cdot \hat{\eta}$.

7. Convert by the inverse Fourier transform these vectors back to the real space, in order to calculate the inner products $\eta \cdot A \eta$, $\eta \cdot B \eta,(A \eta)^{2}$ and $(B \eta)^{2}$.

8. Transfer the obtained vectors to the Fourier space, and apply again operators $A$ and $B$ to obtain summands $\widehat{A(\eta A \eta)}$ and $\widehat{B(\eta B \eta)}$.

9. Now sum up all the terms according to (33) and make the last application of the operator $A$, to obtain the right hand side of the equation.

10. Apply aliasing by cutting away short-waves part of the bandwidth. Usually two thirds of the wave numbers are forced to be zero. 
11. Using the equation (33), calculate a new vector $\eta^{+}$with the RungeKutta algorithm.

12. Repeat from step 5 .

As regards the aliasing step, it is done in order to suppress the inevitable high-frequency mode generation in the nonlinear model. Without this the simulation can blow up.

All the Fourier transforms in the code are performed with the Fast Fourier Transform (FFT), thus the resulting numerical code shows really good performance. For the vast majority of applications the whole calculations can be performed faster than real-time. And the progress in GPU-computing allows to go even further in the speed optimization of the numerical code, as the FFT operation is performed exceptionally quick on modern GPU processors.

\subsubsection{Highest Stokes wave test case}

Looking for the existence and shape of steady waves was an important motivation for research in the nineteenth century (the search for the 'wave of elevation') and continued in the twentieth century. The main motivation in the 1895 paper of Korteweg and de Vries was to investigate this existence matter. The existence and explicit formulation of steady finite energy solutions (later called solitons) and periodic (cnoidal) waves was their contribution to this issue, providing a positive answer about existence in the approximation of the one-way directional $\mathrm{KdV}$-equation. Existence of periodic waves (for the full wave equation) has been investigated in the twentieth century by many scientists; see for instance Craig et al. [3]. Of relevance for this subsection are approximations of the periodic wave shapes using expansion methods (in amplitude, Fourier modes, etc.) as have been derived to a high degree of accuracy by Rienecker \& Fenton [27].

Among the steady periodic solutions, one is exceptional. It occurs on infinitely deep water, and, while the other profiles are smooth, this special one, the Highest Stokes Wave (HSW), is a Stokes wave with a profile that has a corner of 120 degrees in the crest. It was shown recently by Rainey \& Longuet-Higgins [26] that in a good approximation, HSW has the profile of a catenary, i.e. can be written as a hyperbolic cosine curve in between two successive crests. The appearance of a cornered profile indicates a certain singularity in the governing description, since any non-smoothness in an initial profile will be resolved by dispersion in regular dispersive nonlinear equations with differential operators. General Hamiltonian theory about Relative Equilibria (RE) implies that by looking for extremizers of the Hamiltonian $H$ on level sets of the momentum $M$, the extremizers are the profiles of steady waves, and that the multiplier in the governing equation $\delta H=\mu \delta M$ is 
the speed of that profile. We will use this characterization in the next subsection to characterize Stokes waves with the AB-model. Since the $\mathrm{AB}$-equation can also describe waves on infinitely deep water, we will consider that case and investigate the HSW.

AB-relative equilibria (AB-RE) and the highest stokes wave (HSW). Below we formulate the RE-problem and calculate the profiles and speeds. We show that these RE are very close to the set of Rienecker \& Fenton solutions for all values of the momentum until values close to the HSW value. We will also show how well the HSW is approximated. All these results confirm that, at least for these steady solutions, the approximate Hamiltonian and Momentum are rather accurate, providing relatively simple approximations of these integrals of the full surface wave problem. The explicit expression may make these integrals also useful for further advanced functional analytic investigations.

We consider periodic waves of fundamental period $2 \pi$ and zero average $\int \eta d x=0$. Taking the crest at $x=0 \bmod (2 \pi)$, we look for solutions of the RE-equation in the form of a truncated Fourier series. With $a$ an amplitude parameter, we look for solutions as

$$
\eta_{N}=a \cos (x)+a^{2}\left[\sum_{k=2}^{N} \beta_{k} \cos (k x)\right] .
$$

Restricting the Hamiltonian and Momentum to this set, we arrive at functions $H_{N}$ and $M_{N}$ of $p=\left(a, \beta_{2}, \ldots, \beta_{N}\right)$ and the RE-equation becomes

$$
\nabla_{p} H_{N}=\mu_{N} \nabla_{p} M_{N},
$$

which corresponds to the Galerkin projected equation $\delta H=\mu \delta M$. Splitting the Hamiltonian in a quadratic and cubic part, $H_{A B}=H^{(2)}+$ $H^{(3)}$ it can be observed that $H^{(3)}(a \cos (k x))=0$, which implies that the energy for Fourier expansions as above does not contain terms of third order $H_{A B}\left(a \cos x+a^{2} v\right) / g=\frac{1}{2} a^{2}+O\left(a^{4}\right)$. Also, since $\delta H^{(3)}(a \cos (k x))=$ $\frac{1}{2} g a^{2} \cos (2 k x)$, the second order Stokes contribution appears immediately: $\delta H_{A B}(a \cos x) / g=2 a \cos x+\frac{1}{2} a^{2} \cos 2 x$.

In Fig. 4 we show in the Momentum-Hamiltonian plane the curve of AB-Relative Equilibria for approximations of Stokes waves with 16 modes; the tangent at a point to this curve is the propagation speed of the RE at that point. Also shown is the one-term approximation of the HSW by Rainey \& Longuet-Higgins, indicated by a dot; for this solution $a k=0.36$. In Fig. 5 the profile is shown of the AB-RE with the same momentum as HSW. Besides the one-term approximation by Rainey \& Longuet-Higgins, the 2 and 16 mode AB-relative equilibria are plotted, together with a zoom-in near the crest. In all these plots, also the results for the Rienecker \& Fenton (RF) solutions with 64 Fourier modes (dashed line) are shown. 

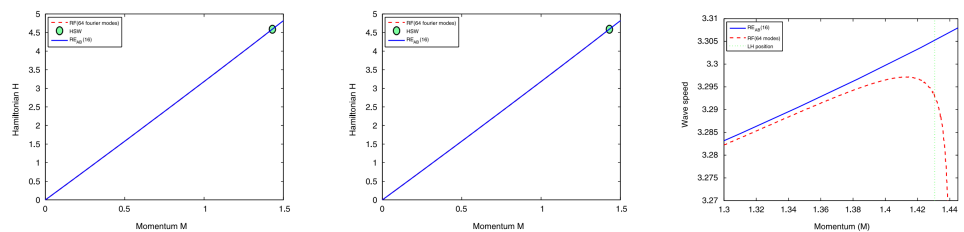

Figure 4: The left plot shows the Momentum (horizontal) versus the Hamiltonian, with the curve of Relative Equilibria of the AB-equation (16 modes: $\left.R E_{A B}(16)\right)$. The dot corresponds to the values of the Highest Stokes Wave (HSW). The curve of the Rienecker \& Fenton approximations with 64 modes (dashed) is hidden but becomes visible when zooming in as done in the middle plot. In the right plot the speed is shown as function of momentum for waves with momentum near the HSW.
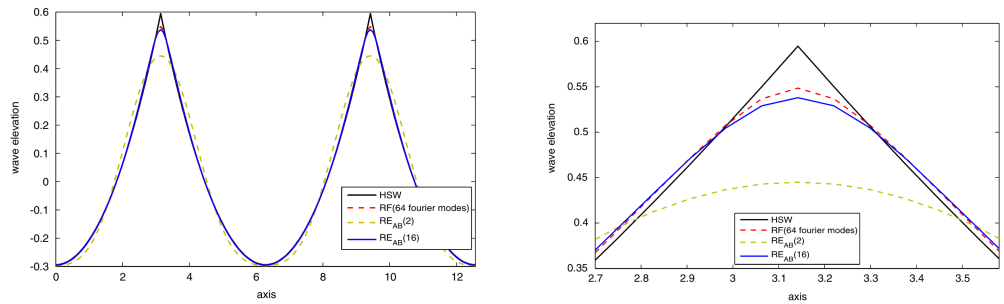

Figure 5: In the left plot the HSW as given by the catenary description, and for the same value of momentum the Relative Equilibria for the ABequation with 2 and 16 modes, and the Rienecker \& Fenton wave with 64 modes are shown. The right plot is a zoom-in near the crest. 
Dynamic simulations. Dynamic simulations have been performed with a very accurate pseudo-spectral implementation (see the previous Subsection 2.2.2), for calculations over more than 100 wavelengths. As expected, all AB-RE travel virtually undisturbed at constant speed for RE's approximated with at least 6 modes. Also the HSW-profile travels for the AB-dynamics with only a small breathing, undisturbed in shape. The speeds of these solutions are equal: the tops remain in a small neighbourhood of each other, with small periodic oscillations.

Discussion and conjecture. The results presented above are for Stokes waves on infinitely deep water, for which no other equation of KdVtype exists but the AB-model. The profiles as well as the dynamics with $\mathrm{AB}$ provide reliable good results, even for the HSW. For parameter values larger than those for the HSW, the numerical scheme does not converge anymore; a similar problem is encountered with the RFsolution technique. This may indicate that such periodic solutions may not exist for these large parameter values.

The results above lead to the conjecture that HSW is a singular solution, not 'connected' to smooth periodic steady waves; for the ABmodel this shows itself from the fact that HSW is not on the AB-curve of RE. The conjecture is supported by the results in $[7,8]$ for the NLSand KdV-equation that maximal crest solutions for prescribed Momentum and Hamiltonian are 'cornered' REs, also in a (one-sided) neighbourhood of the RE-curve. A similar analysis for the AB-equation has not been done yet, but could give more substance to the claim that HSW is isolated and has just 'accidentally' the simple steady dynamics, which means that dispersion does not eliminate the corner as in other cases.

\subsection{INFLUX IN SIGNALLING PROBLEM}

When a new wave model is being built, the traditional approach is to solve the Initial Value Problem first. It can happen that this is enough for the application range of this particular model. However, very often applications require to incorporate boundary conditions into the model. It turns out that introducing robust boundary conditions can be a non-trivial task.

Singalling problem appears naturally in a number of real life applications, in particular, for waves simulated in a hydrodynamic laboratory. A typical one-dimensional laboratory basin layout [29] is shown in Figure 6 . There is a wave flap which generates signal $s(t)$ on the left, and an artificial beach on the right, which damps the incoming waves. Generated waves propagate from left to right, forming a wave field $\eta(x, t)$. It is a task of a model, given a signal, to predict the wave field inside the basin. 


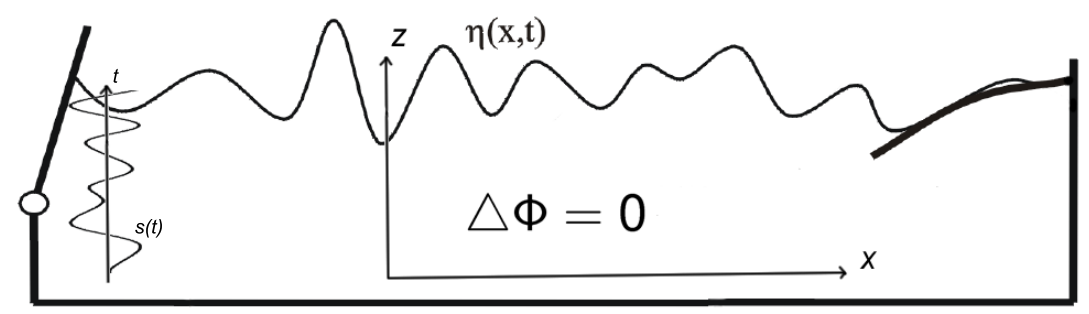

Figure 6: A typical one-dimensional laboratory basin layout. The signal $s(t)=$ $\eta(x=0, t)$ is excited by the wave flap on the left, generated waves propagate to the right, where they are damped by the artificial beach.

It is understandable that in our numerical simulations we need some means of providing an influx, i.e. to excite waves in some point of the spatial domain. This is especially challenging in a spectral setting of the AB-equation, because it does not accept standard types of boundary conditions. The area wave generation described below was first derived for the AB-model. But according to our experience, the described way of influxing is the best for the VBM, too.

We make use of the linear uni-directional equation

$$
\partial_{t} \eta+i \Omega \eta=0
$$

with an operator $\Omega$ defining the dispersion relation $\omega=\Omega(k)$, where $\omega$ is the angular frequency, $k$ is the wave number, and we use $\Omega$ for both the operator and its symbol. Waves in this model propagate to the right, and we will consider only the spatial domain to the right of the origin: $x>0$.

The solution of the equation (34) is

$$
\eta(x, t)=\int \widehat{\eta_{0}}(k) e^{i(k x-\Omega(k) t)} d k=\int \check{s}(\omega) e^{i(K(\omega) x-\omega t)} d \omega,
$$

where we use notation $\hat{\eta_{0}}(k)$ for the spatial Fourier transform of the initial profile $\eta(x, t=0), \breve{s}(\omega)$ for the temporal Fourier transform of the signal at the origin $s(t)=\eta(x=0, t)$, and $K(\omega)$ is the inverse of the dispersion relation function: $k=K(\omega)=\Omega^{-1}(\omega)$. To reduce clutter, we omit $2 \pi$-factors which will cancel out eventually. It follows from the expression (35) that the initial value and the signal are related as $\hat{\eta}_{0}(k) d k=\breve{s}(\omega) d \omega$, via the group velocity factor $V(k)=d \omega / d k$. That is, changing $\omega$ to $k$ and using definition of the Fourier transform in (35), we obtain

$$
\eta(x, t)=\iint s(\tau) e^{i(k x-\Omega(k)(t-\tau))} V(k) d \tau d k .
$$

This can be interpreted as (different harmonics in) the signal $s(\tau)$ being translated (with different phase velocities) from the origin to the point 
$x$, forming the wave elevation function $\eta(x, t)$ in that point. It is clear that in the uni-directional equation (34), where waves are propagating to the right only, the signal $\eta$ in the point $x$ will be defined by the history of the signal $s$, i.e. by $s(\tau)$ with $\tau<t$. So, for $x>0$ we can change upper boundary of the integral:

$$
\begin{aligned}
\eta(x, t) & =\iint^{t} s(\tau) e^{i(k x-\Omega(k)(t-\tau))} V(k) d \tau d k \\
& =\int V(k) e^{i k x} \int^{t} s(\tau) e^{-i \Omega(k)(t-\tau)} d \tau d k .
\end{aligned}
$$

Now let us take the forced version of the linear uni-directional equation with the same dispersion operator:

$$
\partial_{t} \eta+i \Omega \eta=\gamma(x) \cdot s(t),
$$

where initial profile $n(x, t=0)$ is zero, signal $s(t)$ is the same as previously, and $\gamma(x)$ is an unknown spatial function. We want to find such forcing profile $\gamma(x)$ that the source term $\gamma(x) \cdot s(t)$ will excite the same wave field $\eta(x, t)$ as in the previous model (34).

The solution of the inhomogeneous equation (38) is

$$
\eta(x, t)=\int^{t} \gamma(x) s(\tau) e^{-i \Omega(t-\tau)} d \tau=\int \hat{\gamma}(k) e^{i k x} \int^{t} s(\tau) e^{-i \Omega(k)(t-\tau)} d \tau d k .
$$

Comparing this expression to the formula (37), we observe that $\hat{\gamma}(k)=$ $V(k)$. Then we immediately arrive at the result, stating that the area generation function $\gamma(x)$ is the inverse Fourier transform of the group velocity:

$$
\gamma(x)=\int V(k) e^{i k x} d k
$$

In every model, where an analytic dispersion relation is calculated, we can apply the area wave generation by adding the source term $\gamma(x) \cdot s(t)$ to the $\eta$-equation, where $\gamma$-function is calculated according to (40) and depend on the group speed. For instance, in the VBM system (15) we add this term to the right hand side of the first equation. The same is true for the AB-equation (30), but for the pseudo-spectral implementation (33) we have to transfer the $\gamma$-function (back) to the Fourier space.

As in all subsequent chapters we simulate a signalling problem, we make extensive use of such influxing for both the VBM and the ABequation. In the latter case we normally use the exact dispersion relation. The shapes of $\gamma$-functions for the exact dispersion (22) and dispersion of the VBM with a parabolic profile (see Subsection 3.2.2) are shown in Figure 7 . Notice, both $\gamma$-functions are infinite in zero, but unlike the exact $\gamma$-function, the VBM counterpart has a delta-function 


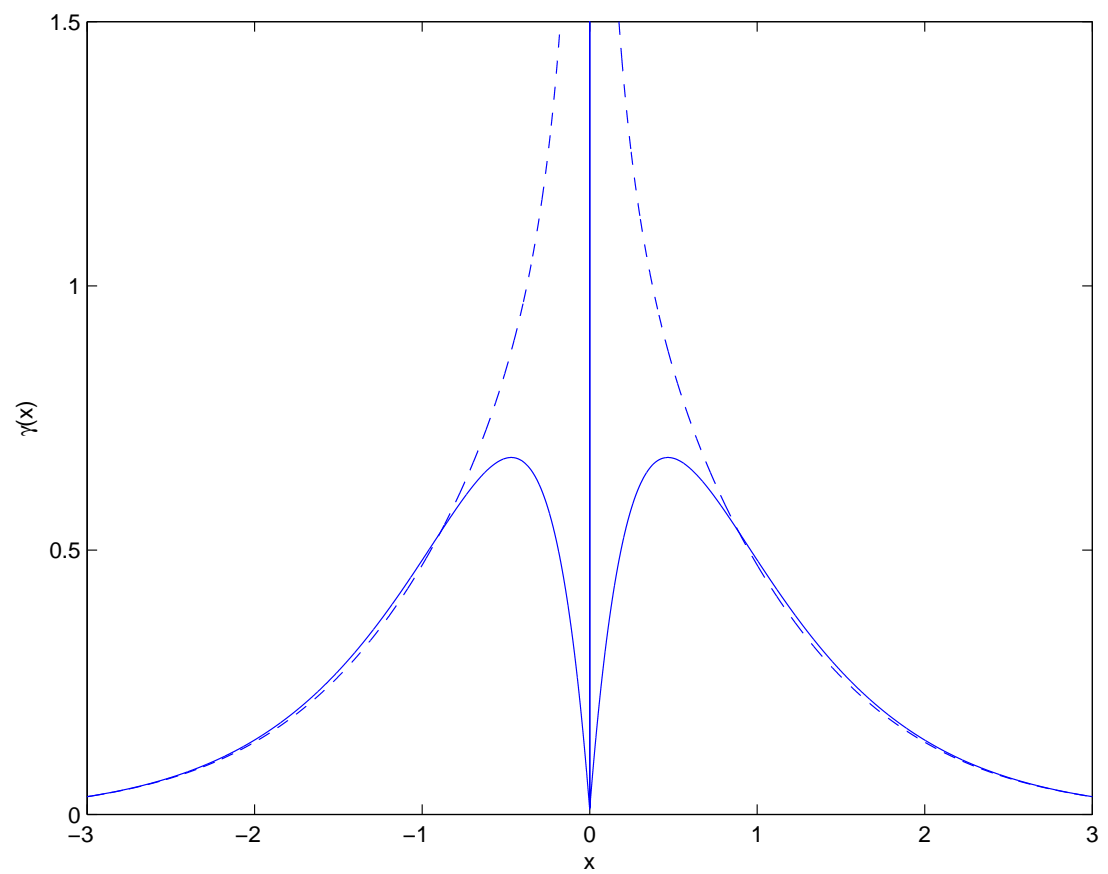

Figure 7: The shapes of $\gamma$-functions for the exact dispersion (dashed) and dispersion of the VBM with a parabolic profile (solid). The depth $h_{0}=1 \mathrm{~m}$. 
in the origin. This is due to the non-zero limit of the group speed for $k \rightarrow \infty$; see expression (49) in the following Chapter.

For the evaluation of the described embedded boundary condition, see $[4,28]$. More general form of the area wave generation is derived there as well, which allows, for instance, the point source influxing.

\subsection{CONCLUSIONS AND REMARKS}

The Variational Boussinesq Model (VBM) is obtained via the Luke's action principle, constricting the potential flow to certain families of functions. Thus, it has decreased dimensionality comparing to the full problem and other nice properties like conservation of energy. See Subsection 2.1.6 and also Chapter 6 for the description of various advantages of the VBM.

Brief introduction into a few variants of the VBM has been presented. These variants will be extensively exploited throughout the following chapters. We mentioned several types of the dispersion, which can be used in the VBM, and postponed the detailed investigation of dispersion choice consequences to the next chapters.

We also reviewed the AB-equation, which will serve as the main instrument of comparison. As the uni-directional AB-model allows to choose the exact dispersion and also has good nonlinear properties, this comes in handy when judging about performance of the VBM.

For both models we gave the description of the numerical codes, being the FEM implementation for the VBM and the pseudo-spectral code for the AB-equation. Demonstration of the numerical performance of the latter model was done, using the example of the Highest Stokes Wave. As all the test cases simulated in the subsequent chapters belong to the signalling problem kind, we found it useful to recall the derivation of the embedded boundary condition we use in a signalling problem. The derived boundary condition is capable of providing an influx in any dispersive model. It turns out it requires knowledge of the analytical dispersion relation of the model. 

[1] B. Benjamin, Lectures on nonlinear wave motion, in: Nonlinear Wave Motion, Lectures in Applied Mathematics 15 (1974) AMS, Providence.

[2] L.J.F. Broer, On the Hamiltonian theory of surface waves, Appl. Sci. Res. 30 (1974) 340.

[3] W. Craig, P. Guyenne, H. Kalisch, Hamiltonian long-wave expansions for free surfaces and interfaces, Comm. Pure Appl. Math. 58 (2005) 1587-1641.

[4] D. Adytia, Coastal Zone Simulations with Variational Boussinesq Modelling, Ph. D. Thesis, Univ. of Twente, Enschede, The Netherlands (2012) 114 pp., ISBN-13: 978-90-365-3351-5.

[5] D. Adytia, E. van Groesen, The Variational 2D Boussinesq model for wave propagation over a shoal, in: International Conference on Developments in Marine CFD, 18 - 19 November 2011, Chennai, India.

[6] D. Adytia, E. van Groesen, Optimized Variational 1D Boussinesq modelling of coastal waves propagating over a slope, Coast. Eng. 64 (2012) 139-150.

[7] E. van Groesen, Andonowati, Finite energy wave signals of extremal amplitude in the spatial NLS-dynamics, Phys. Lett. A 357 (2006) 86-91.

[8] E. van Groesen, Andonowati, Extremal periodic wave profiles, Nat. Hazards Earth Syst. Sci. 7 (2007) 33-40.

[9] E. van Groesen, Andonowati, Variational derivation of KdV-type of models for surface water waves, Phys. Lett. A 366 (2007) 195201.

[10] E. van Groesen, Andonowati, L. She Liam, I. Lakhturov, Accurate modelling of uni-directional surface waves, J. Comput. Appl. Math. 234 (2010) $1747-1756$.

[11] E. van Groesen, Jaap Molenaar, Continuum Modeling in the Physical Sciences, SIAM, Mathematical Modeling and Computation Vol. 13, 2007, 236 pp.; ISBN-13: 978-o-898716-25-2.

[12] R. Johnson, A Modern Introduction to the Mathematical Theory of Water Waves, (1997) Cambridge Univ. Press. 
[13] J. T. Kirby, Boussinesq models and applications to nearshore wave propagation, surfzone processes and wave-induced currents, in Advances in Coastal Modelling (Elsevier) 67 (2003) 1-41.

[14] G. Klopman, M. W. Dingemans, E. van Groesen, A variational model for fully non-linear water waves of Boussinesq type, in: Proceedings of the International Workshop on Water Waves and Floating Bodies, 2005.

[15] G. Klopman, M. W. Dingemans, E. van Groesen, Propagation of wave groups over bathymetry using a variational Boussinesq model, in: Proceedings of the International Workshop on Water Waves and Floating Bodies, 2007.

[16] G. Klopman, M. W. Dingemans, Reflection in variational models for linear waves, Wave Motion 47 (2010) 469-489.

[17] G. Klopman, E. van Groesen, M. W. Dingemans, A variational approach to Boussinesq modelling of fully nonlinear water waves, J. Fluid Mech. 657 (2010) 36-63.

[18] G. Klopman, Variational Boussinesq Modelling of Surface Gravity Waves over Bathymetry, Ph. D. Thesis, Univ. of Twente, Enschede, The Netherlands (2010) 114 pp., ISBN-13: 978-90-365-3037-8.

[19] D. Korteweg, G. de Vries, On the change of form of long waves advancing in a rectangular canal, and on a new type of long stationary waves, Phil. Mag. 39 (1895) 422-443.

[20] I. Lakhturov, D. Adytia, E. van Groesen, Optimized Variational ID Boussinesq Modelling for broad-band waves over flat bottom, Wave Motion 49 (2012) 309-322.

[21] I. Lakhturov, E. van Groesen, Optimized Variational Boussinesq Modelling; part 1: Broad-band waves over flat bottom, Internal report, University of Twente, The Netherlands (2010). See http:// eprints . eemcs . utwente.nl/.

[22] J.C. Luke, A variational principle for a fluid with a free surface, J. Fluid Mech. 27 (1967) 395-397.

[23] P. A. Madsen, H. A. Schäffer, A review of Boussinesq-type equations for gravity waves, in: World Scientic, Advances in Coastal and Ocean Engineering (World Scientic) 5 (1999) 1-95.

[24] P. A. Madsen, D. R. Fuhrman, High-order Boussinesq-type modelling of nonlinear wave phenomena in deep and shallow water, in: Advances in numerical simulation of nonlinear water waves (World Scientic) 5 (2010) 245-285. 
[25] J.W. Miles, On Hamilton's principle for surface waves, J. Fluid Mech. 83 (1977) 153-158.

[26] R. Rainey, M. Longuet-Higgins, A close one-term approximation to the highest Stokes Wave on deep water, Ocean Eng. 33 (2006) 2012-2024.

[27] M. Rienecker, J. Fenton, A Fourier approximation method for steady water wave, J. Fluid Mech. 104 (1981) 119-137.

[28] L. She Liam, D. Adytia, and E. van Groesen, On internal wave generation for 1 - and $2 \mathrm{D}$ dispersive surface wave models, In preparation.

[29] J. Westhuis, The numerical simulation of nonlinear waves in a hydrodynamic model test basin, Ph. D. Thesis, Univ. of Twente, Enschede, The Netherlands (2001) 244 pp., ISBN: 90-365-1575-0.

[30] V.E. Zakharov, Stability of periodic waves of finite amplitude on the surface of a deep fluid, J. Appl. Mech. Tech. Phys. 2 (1968) 190194 . 

The Variational Boussinesq Model (VBM) for waves above a layer of ideal fluid conserves mass, momentum, energy, and has decreased dimensionality compared to the full problem. It is derived from the Hamiltonian formulation via an approximation of the kinetic energy, and can provide approximate dispersion characteristics. Having in mind a signalling problem, we search for optimal dispersive properties of the 1-D linear model over flat bottom and, using finite element and (pseudo-) spectral numerical codes, investigate its quality. For the optimization we restrict to the class of potentials with hyperbolic vertical profiles that are parametrized by the wavenumber. The optimal wavenumber is obtained by minimizing the kinetic energy for the given signal and produces good results for two realistic test cases. Besides this kinetic energy principle we also consider various ad-hoc least square type of minimization problems for the error of the phase or group velocity. The test cases are two examples of focussing wave groups with broad spectra for which accurate experimental data are available from MARIN hydrodynamic laboratory. To determine the quality of an 'optimized' wavenumber for the governing dynamics, we use accurate numerical simulations with the $\mathrm{AB}$-equation to compare with VBM calculations for the whole range of possible wavenumbers. The comparison includes the errors in the signal at the focussing position, as well as the integrated errors of maximal and minimal wave heights along a spatial and temporal interval that is symmetric around the focussing event.

This chapter comprise the contents of the technical report [8].

\subsection{INTRODUCTION}

The Variational Boussinesq Model (VBM) is based on the fact that the surface wave evolution can be described as an infinite dimensional Hamiltonian system. The canonical variables are the surface elevation $\eta$ and the fluid potential $\phi$ at the free surface. The Hamiltonian is the total energy, of which the kinetic energy is given by

$$
K(\phi, \eta)=\iint_{-h}^{\eta} \frac{1}{2}|\nabla \Phi|^{2} d z d x,
$$

where the fluid potential $\Phi$ satisfies the Laplace equation in the interior, the impermeability condition at the bottom $z=-h$ and the prescribed value $\Phi=\phi$ at the free surface $z=\eta$. Here we use notation $K$ for 
the kinetic energy functional, different than previously (in expression (3) where we used $K$ for the kinetic energy density). In this chapter we consider the bottom to be flat; the case of varying bottom will be dealt with in Chapter 5 . This potential $\Phi$ has the extremal property that it minimizes the kinetic energy over all potentials that satisfy the prescribed surface value $\phi$. This minimization property of the kinetic energy will be exploited further on in an essential way to obtain the best dispersive properties.

Since the Laplace problem cannot be solved explicitly for nontrivial $\eta$, the kinetic energy has to be approximated to make the model useful for numerical simulations.

In this chapter, just as in $[5,6,7]$, we choose to take the approximation that follows by writing $\Phi$ as a one-term perturbation of the surface potential:

$$
\Phi(x, z)=\phi(x)+F(z) \psi(x),
$$

requiring $F(\eta)=0$ along with the bottom impermeability condition. The vertical profile function $F$ has to be chosen in advance, and the function $\psi$ at the free surface becomes an additional variable for which an additional elliptic equation has to be solved together with $\eta$ and $\phi$.

The dispersion relation of the resulting dynamical system depends (strongly) on the choice of the function $F$. The choice $F=0$ leads to the shallow water equations (SWE) with no dispersion. From linear theory, the fluid potential of a small amplitude harmonic wave with wave number $\kappa$ can be exactly represented by the choice

$$
F(z)=\frac{\cosh \kappa(z+h)}{\cosh \kappa(\eta+h)}-1 .
$$

But for non-harmonic waves, or when nonlinearity is essential, the form (43) can at best be approximative.

In this chapter we address the question for which choice of $F$ one gets the best dispersive properties. We restrict to the linearized equations, and consider the signaling problem for wave fields in $1 \mathrm{D}$ with broad-band spectra. We will keep the form (43) as Ansatz, but allow the value of $\kappa$ to be chosen in an optimal way. Intuitively, the optimal $\kappa$ will be some averaged wave number, the value of which will depend on properties of the wave field, in particular on the initial profile or the initial signal.

Since the dispersion relation related to the choice of $F$ in (43) will depend on $\kappa$, it is natural to choose $\kappa$ in such a way that the difference with the exact dispersion relation, given by

$$
\omega=\Omega_{e x}(k)=\operatorname{sign}(k) \cdot \sqrt{g k \tanh k h},
$$

is as small as possible for relevant wave numbers. But it is not obvious which norm to choose for measuring the difference. Moreover, it is 
not simple to know what the effect is of differences in the dispersion relation on the behaviour of the wavefields.

In the following we will show that a natural choice for $\kappa$ is obtained by minimizing the kinetic energy for the given initial time signal. This optimization is well-founded by the minimality property of the kinetic energy. Nevertheless we will also consider some ad-hoc least square formulations. These are of the form of minimizing the phase speed error or the group speed error in the $L_{2}$-norm, weighted with the initial spectrum. It will be shown that these optimal values give almost the same optimization result for the two test cases considered here.

The two test cases are focussing wave groups that have been generated and measured at MARIN hydrodynamic laboratory, Wageningen, the Netherlands. Instead of the MARIN numbering 109001 and 101013, we will refer to these cases as the mild and the strong focussing group, denoted by $\mathrm{mFG}$ and $\mathrm{sFG}$ respectively.

In order to qualify the VBM results, we compare the evolutions with simulations using the linear version of the $\mathrm{AB}$-equation $[2,3]$. These $\mathrm{AB}$-simulations have exact dispersive effects, and turn out to be very accurate when compared to the point measurements of MARIN. With these accurate simulations, we compare the VBM calculations. We compare the time signal at the focussing point, but also the whole spatial evolution of the focussing and de-focussing behaviour as represented by the maximal and minimal temporal amplitudes. It will turn out that in all cases the optimal $\kappa$ is far away from the peak-wave number, which could have been a first guess for an optimum.

The outline of the chapter is as follows. In Section 3.2 we present the dynamic equations, and investigate the dispersion relation in its dependence on $\kappa$. In particular, we show that for each $\kappa$, all sufficiently short waves have the same, finite propagation speed; this erroneous behaviour is an inevitable consequence of the fact that we represent the fluid potential as in (42).

In Section 3.3 we derive the kinetic energy optimization principle to calculate the optimal $\kappa$-value. In addition, we propose various optimization criteria which could be used as well. In Section 3.4 we describe the two test cases of focussing wave groups and show that the simulations with the linear and non-linear AB-equation are very close to the MARIN experiments. In Section 3.5 we calculate the optimal $\kappa$-values according to the previously proposed optimization criteria, and we present the comparison between the optimal VBM calculations and the AB-simulations. In Section 3.6 we provide some remarks and conclusions.

It should be remarked that the analysis in this chapter is concentrated on the errors caused by the modelling process, and not on numerical accuracy errors. The numerical simulations are performed for 
the exact dispersion with a spectral code; for the VBM simulation we used a FE-implementation with sufficiently fine grid.

\subsection{THE VBM DISPERSION RELATIONS}

As written in the introduction, every Variational Boussinesq model is obtained via approximation of the kinetic energy. We accomplish this by approximating the fluid potential $\Phi$ by the expression (42). Since we are studying the dispersive properties in this thesis, we will restrict to linearized equations. This is obtained by replacing in (4I) the vertical integration interval till the still water level 0 instead of till the surface elevation $\eta$, and putting $\eta=0$ in (43).

The kinetic energy is then given by

$$
K \approx \frac{1}{2} \int\left[h\left(\partial_{x} \phi\right)^{2}+\alpha\left(\partial_{x} \psi\right)^{2}+\gamma \psi^{2}+2 \beta \partial_{x} \phi \partial_{x} \psi\right] d x
$$

with integral coefficients $\alpha, \beta$ and $\gamma$ that are given by

$$
\alpha=\int_{-h}^{0} F^{2} d z, \quad \beta=\int_{-h}^{0} F d z, \quad \gamma=\int_{-h}^{0}\left(F^{\prime}\right)^{2} d z .
$$

Based on Luke's variational principle $[4,9]$ (see also $[1,12,13])$, variations of the following Lagrangian should be equal to zero.

$$
\delta \mathcal{L}=\delta \int\left(\int \phi \partial_{t} \eta d x-K-P\right) d t=0,
$$

where $P=\frac{1}{2} \int g \eta^{2} d x$ is the potential energy.

Variations of the Lagrangian with respect to $\phi, \eta$ and $\psi$ give the following system of PDEs, which we call the Linear Variational Boussinesq Model,

$$
\left\{\begin{array}{l}
\partial_{t} \eta=-h \partial_{x}^{2} \phi-\beta \partial_{x}^{2} \psi \\
\partial_{t} \phi=-g \eta \\
-\alpha \partial_{x}^{2} \psi+\gamma \psi=\beta \partial_{x}^{2} \phi .
\end{array}\right.
$$

The coefficients $\alpha, \beta$ and $\gamma$ depend on the approximation of the vertical potential profile $F$ of the model. For a parabolic approximation of the profile $F$, taken in [5] and [6], they depend only on depth. For the cosine hyperbolic approximation, on which we concentrate in this chapter, the coefficients depend also on the wave number $\kappa$; the value of this parameter will shortly be determined in an optimal way. 


\subsubsection{VBM dispersion relation}

We obtain an analytic expression for the dispersion of the system (47): we look for harmonic profiles with frequency $\omega(k)$ depending on the wave number $k$ :

$$
\eta=a e^{i(k x-\omega t)}, \quad \phi=b e^{i(k x-\omega t)}, \quad \psi=c e^{i(k x-\omega t)} .
$$

Substituting these profiles into the system (47), we obtain a matrix equation in a form $L \cdot(a, b, c)^{T}=0$, for which non-trivial solutions exist only when $\operatorname{det} L=0$. This gives the dispersion relation is given through the phase velocity $C_{V B M}=\omega / k$, by

$$
C_{V B M}=c_{0} \sqrt{1-\frac{\beta^{2}}{h} \cdot \frac{k^{2}}{\gamma+\alpha k^{2}}}
$$

where $c_{0}=\sqrt{g h}$. Unlike the exact phase speed, this approximation has the nonzero limit for short waves

$$
\lim _{k \rightarrow \infty} C_{V B M}(k)=c_{0} \sqrt{1-\frac{\beta^{2}}{\alpha h}} .
$$

Indeed, this limit is real and nonzero as a consequence of the fact that $\beta^{2} \leqslant \alpha h$ because of Cauchy-Schwarz inequality:

$$
\left(\int_{-h}^{0} F \cdot 1 d z\right)^{2} \leqslant \int_{-h}^{0} F^{2} d z \cdot \int_{-h}^{0} 1 d z,
$$

while equality in this expression is only possible for trivial functions F.

The limit for long waves is, as it should be, $c_{0}=\sqrt{g h}$. The Taylor expansion around $k=0$ yields

$$
\begin{gathered}
\Omega_{V B M} \approx c_{0} k\left[1-\frac{\beta^{2}}{2 h \gamma} k^{2}+\left(\frac{\alpha \beta^{2}}{2 h \gamma^{2}}-\frac{\beta^{4}}{8 h^{2} \gamma^{2}}\right) k^{4}-\right. \\
\left.\left(\frac{\alpha^{2} \beta^{2}}{2 h \gamma^{3}}-\frac{\alpha \beta^{4}}{4 h^{2} \gamma^{3}}+\frac{\beta^{6}}{16 h^{3} \gamma^{3}}\right) k^{6}+O\left(k^{8}\right)\right] .
\end{gathered}
$$

It should be noticed that these expressions are valid for a Variational Boussinesq Model with any vertical potential approximation $F(z)$ in (42), although the integral coefficients (46) will depend on model parameters, e.g. the wave number $\kappa$ as appears in the cosh-approximation (43).

Fig. 8 shows normalized plots of the phase and group speed for the parabolic and the cosine hyperbolic models; for comparison, also the plot of the exact phase and group speed is given, where the group speed is expressed by $V=\frac{d \omega}{d k}$. 


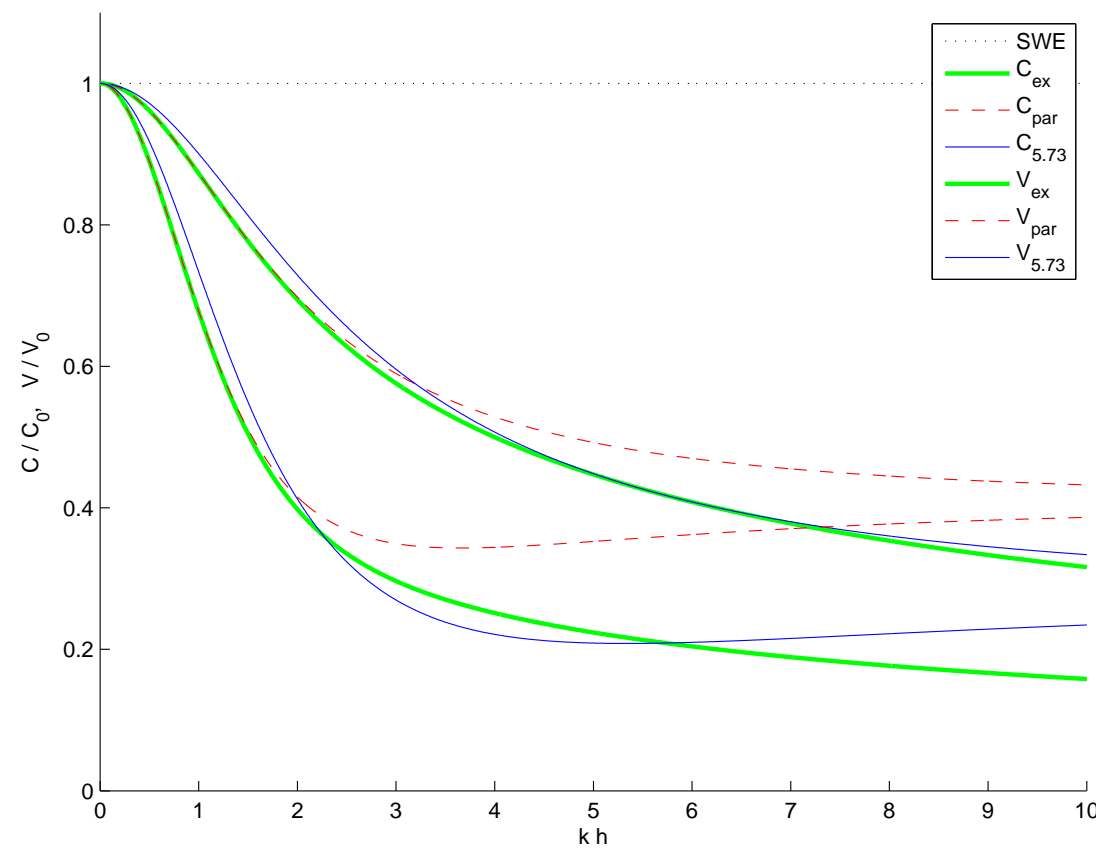

Figure 8: The phase speed (upper curves) and the group speed (lower curves) for different models: the shallow water equation (dotted black), the exact dispersion (thick solid green), the parabolic approximation (dashed red), the cosine hyperbolic approximation with $\kappa=5.73$ (thin blue solid). 


\subsubsection{Parabolic Approximation}

The parabolic approximation for the function $F$ has been extensively discussed by Klopman e.a. $[5,6]$; we will briefly recall the results. The function $F$ in (42) is taken to be

$$
F(z)=(A+1) \frac{z}{h}+A \frac{z^{2}}{h^{2}} .
$$

We set $A=1$ because of the bottom impermeability condition $F^{\prime}(-h)=$ 0 . The surface condition $F(0)=0$ is satisfied as well, and additionally, we can normalize the function so that $F(-h)=-1$. Then the coefficients are given by

$$
\alpha=\frac{8}{15} h, \quad \beta=-\frac{2}{3} h, \quad \gamma=\frac{4}{3} \cdot \frac{1}{h} .
$$

After substitution of $\alpha, \beta$ and $\gamma$ from these formulae in expression (48), one gets the expression by Klopman e.a. [5, 6] (compare to the appropriate expressions in [10, 11]):

$$
\frac{\omega^{2} h}{g}=(k h)^{2} \cdot \frac{1+\frac{1}{15}(k h)^{2}}{1+\frac{2}{5}(k h)^{2}} .
$$

The phase speed $\omega / k$ by this formula has the limit $\sqrt{g h / 6}$ for $k \rightarrow \infty$.

Comparing the Taylor expansion around $k=0$

$$
\Omega_{V B M}^{\text {parab }} \approx c_{0} k\left[1-\frac{1}{6}(k h)^{2}+\frac{19}{360}(k h)^{4}-\frac{193}{10800}(k h)^{6}+O\left((k h)^{8}\right)\right]
$$

to the Taylor expansion of the exact dispersion relation (44)

$$
\Omega_{e x} \approx c_{0} k\left[1-\frac{1}{6}(k h)^{2}+\frac{19}{360}(k h)^{4}-\frac{55}{3024}(k h)^{6}+O\left((k h)^{8}\right)\right],
$$

one can conclude that the dispersion of the VBM equations is correct up to and including the 5-th order for long waves.

\subsubsection{Hyperbolic Cosine Approximation}

According to the linear theory for small-amplitude gravity driven waves on a layer of ideal fluid, the expression (43) leads to the correct fluid potential for harmonic waves with wave number $\kappa$.

The surface condition $F(0)=0$ is satisfied along with the bottom impermeability condition $F^{\prime}(-h)=0$. The integral coefficients $\alpha, \beta$ and $\gamma$ in (46) are:

$$
\begin{gathered}
\alpha(\kappa)=-\frac{3}{2 \kappa} \tanh \kappa h+\frac{1}{2} \frac{h}{\cosh ^{2} \kappa h}+h, \\
\beta(\kappa)=\frac{1}{\kappa} \tanh \kappa h-h, \\
\gamma(\kappa)=\frac{\kappa}{2} \tanh \kappa h-\frac{\kappa^{2}}{2} \frac{h}{\cosh ^{2} \kappa h} .
\end{gathered}
$$


The Taylor expansion of these coefficients around $\kappa=0$ gives

$$
\begin{gathered}
\alpha(\kappa)=h\left[\frac{2}{15}(\kappa h)^{4}-\frac{34}{315}(\kappa h)^{6}+O\left((\kappa h)^{8}\right)\right], \\
\beta(\kappa)=h\left[-\frac{1}{3}(\kappa h)^{2}+\frac{2}{15}(\kappa h)^{4}-\frac{17}{315}(\kappa h)^{6}+O\left((\kappa h)^{8}\right],\right. \\
\gamma(\kappa)=\frac{1}{h}\left[(\kappa h)^{4}-\frac{4}{15}(\kappa h)^{6}+O\left((\kappa h)^{8}\right] .\right.
\end{gathered}
$$

Substituting the coefficient values (55) into the phase and group speed expressions, we observe that whatever the value of $\kappa$ is, the dispersion relation is exact in $k=\kappa$ for both the phase speed and the group speed:

$$
\begin{aligned}
& C_{\kappa}(k)=\left.C_{e x}(k) \quad\right|_{k=\kappa}, \\
& V_{\kappa}(k)=\left.V_{e x}(k) \quad\right|_{k=\kappa} .
\end{aligned}
$$

Remark. We observe from Fig. 8 that it seems that $C_{\kappa} \geqslant C_{e x}$ : the approximate phase velocity is for each wavenumber larger than or equal to the exact phase speed. This is actually true for any approximate VBM and is a direct consequence of the minimization property of the (quadratic) kinetic energy, mentioned in the introduction. Indeed, the kinetic energy can be written for any linear dispersive wave equation as

$$
K(\phi)=\frac{1}{2 g} \int u C^{2} u d x \text { with } u=\partial_{x} \phi,
$$

where $C$ is the phase velocity. For the exact dispersion with $C_{e x}$ and an approximate VBM model, such as $C_{\mathcal{K}}$ in the hyperbolic approximation, we have

$$
\begin{aligned}
K(\phi) & =\min _{\Phi=\phi \text { at } z=0} \frac{1}{2} \iint|\nabla \Phi|^{2} d z d x=\frac{1}{2 g} \int u C_{e x}^{2} u d x \\
& \leqslant \frac{1}{2} \iint\left|\nabla \Phi_{V B M, \kappa}\right|^{2} d z d x=\frac{1}{2 g} \int u C_{\mathcal{K}}^{2} u d x .
\end{aligned}
$$

Since this holds for each $\phi$ (each $u$ ) we conclude that $C_{e x}^{2} \leqslant C_{\mathcal{K}}^{2}$ for each $\kappa$. For the hyperbolic profile we have

$$
C_{e x}(\kappa)=C_{\kappa}(\kappa) \text {, and } C_{e x}(k)<C_{\kappa}(k) \text { for } k \neq 0, \kappa \text {. }
$$

\section{$3 \cdot 3$ OPTIMIZATION CRITERIA}

As stated above, it is not clear in advance how to choose an optimal value of $\kappa$. To illustrate the problem, in Fig. 9 we present the power spectrum $S(\omega)$ of a time signal of the wave at one position for the two test cases that we will consider in the next section.

In the same figure we plotted the graph of the phase velocity $C_{t}(\omega)$; here we used the subscript $t$ to indicate that we consider the phase 
velocity as a function of frequency. Hence, for given dispersion relation $\omega=\Omega(k)$, we consider the inverse $k=K(\omega)$ and define $C_{t}$ as

$$
C_{t}(\omega)=C(K(\omega))=\frac{\omega}{K(\omega)} .
$$

When using the exact dispersion relation $\omega=\Omega_{e x}(k)$, we will specify this by writing $C_{t, e x}$. The same figure shows a plot of the group velocity $V_{t}(\omega)$, where again the subscript $t$ indicates that we take the group velocity as a function of frequency. It is defined as $V_{t}(\omega)=V(K(\omega))$.

When using the VBM-hyperbolic profile, we have to transfer a choice for $\kappa$ to the frequency domain. Since the exact and the VBM-hyperbolic dispersion relation coincide at $\kappa, \Omega_{e x}(\kappa)=\Omega_{\kappa}(\kappa)$, the transformation is independent of this choice, and we find a unique value $v=\Omega_{e x}(\kappa)=$ $\Omega_{\kappa}(\kappa)$ corresponding to $\kappa$. Therefore we will write $C_{t, v}$ to denote the VBM-phase speed as function of the frequency:

$$
C_{t, v}(\omega)=\frac{\omega}{K_{\kappa}(\omega)} \text { with } v=\Omega_{\kappa}(\kappa) .
$$

The difference between the two phase speeds is denoted by $\Delta C_{t, v}$

$$
\Delta C_{t, v}(\omega)=C_{t, v}(\omega)-C_{t, e x}(\omega)
$$

Since the derivatives of the exact dispersion relation $\Omega_{e x}(k)$ and the hyperbolic one coincide in the point $\kappa$, the group speed error

$$
\Delta V_{t, v}(\omega)=V_{t, v}(\omega)-V_{t, e x}(\omega)
$$

is also zero for $\omega=v$. It turns out that there is another zero value, and the frequency for which the group speeds are the same is quite close to the peak frequency of the shown spectra (for the chosen value of $v$ ).

In the figure we also plot the error for the phase and group velocity as function of $v$. Actually, the specific value of $v$ is not relevant for the present reasoning; the chosen values are actually the optimal choices according to the kinetic energy minimization to be defined below.

This figure illustrates the problem how to choose an optimal value $v$. Intuitively, we would like the approximate velocity to be accurate, i.e. small $\Delta C$ or small $\Delta V$, where the spectrum is large, but for applications with a broad spectrum as in this example, the best value is not obvious. Besides that, the problem is made even more intricate because we have only very limited intuition what the effect of changes in phase or group speed is on the actual evolution of the waves.

The error $\Delta V$ is much larger than $\Delta C$ in the tail of the spectrum close to $\omega=10$ : the error $\Delta V$ is comparable to the actual value, while the error $\Delta C$ is approximately $20 \%$ near $\omega=10$. Since $\Delta V$ vanishes also in a point close to the peak frequency, we observe that an optimal $v$-value gives a group speed curve that has minimal error over a rather large 

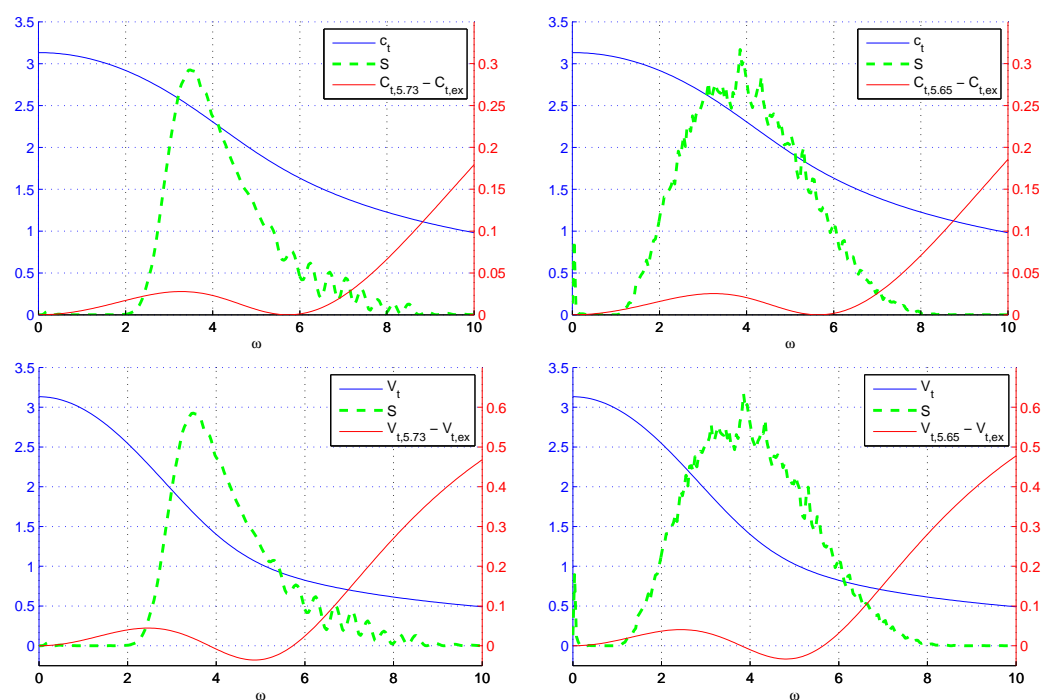

Figure 9: The broad-band signal spectrum (with convenient normalization, green, dashed) is shown as function of frequency for the two MARIN test cases to be studied below: at the left for the mFG and at the right for the sFG. In the upper plots the exact phase velocity and in the lower plots the exact group speed are given (blue, solid). For both the error between the exact dispersion and the VBM for some (optimal) value of $v$ is given (red, solid). 
frequency range, but the error increases much faster than the error in phase speed for higher frequencies.

In this chapter we first investigate in Subsection 3.3.1 some ad-hoc, but reasonable optimization criteria. Then we will use the criterion of kinetic energy minimization in Subsection 3.3.2. These optimization criteria will be used in the next section to determine the quality of the resulting dynamics, and to verify that the kinetic energy minimizer is the best choice.

\subsubsection{Weighted least square formulations}

In this subsection we will consider several ad-hoc least square formulations, each of which aims to reduce the velocity error over the whole relevant frequency interval. The methods can be formulated as a temporal optimization problem

$$
\operatorname{Err}_{v}:=\int\left|\Delta W_{v}(\omega)\right|^{2} \rho_{t}(\omega) d \omega \rightarrow \min _{v}
$$

or a spatial optimization problem

$$
\operatorname{Err}_{\kappa}:=\int\left|\Delta W_{\mathcal{K}}(k)\right|^{2} \rho_{s}(k) d k \rightarrow \min _{\mathcal{K}}
$$

where $\rho_{t}$ and $\rho_{s}$ denote temporal and spatial weight functions to be chosen. $\Delta W$ is the difference of the velocity $W$ in the VBM model and the exact velocity: $\Delta W=W_{V B M}-W_{e x}$, where for $W$ we will consider the phase or the group velocity. Be warned that we use rather sloppy, but efficient, notation: $\Delta W_{v}(\omega)=\Delta W_{\kappa}(k)$ for $\omega=\Omega_{\kappa}(k)$ and $v=\Omega_{\kappa}(\kappa)$. Also note that $\rho_{t}(\omega) d \omega=\rho_{t}\left(\Omega_{\kappa}(k)\right) V_{k}(k) d k$ so that the formulations are closely related, but that if $\rho_{t}=\rho_{s}$ formulations differ by the group velocity. Different cases arise by making different specific choices for the velocity and for the weight functions. In principle also the integration boundaries can be chosen, but for the confined - yet broad-band - examples we will consider, it is most appropriate to take the integration over the total real line, which we will do in the following.

We will consider two choices for the speed: the phase velocity $C$ and the group velocity $V$. As weight function we will take the power spectrum of (the influx of) the wave field under consideration. This leads us to four possible criteria, for $W=C$ (the phase velocity) or $W=V$ (the group velocity), in the expressions $\operatorname{Err}_{v}=\int\left|\Delta W_{v}(\omega)\right|^{2} S(\omega) d \omega$ or $E r r_{\kappa}=\int\left|\Delta W_{\kappa}(k)\right|^{2} S(k) d k$. Observe that for given $S(\omega)$ we have $S(k)=S\left(\Omega_{V B M}(k)\right)$. Hence, the difference between the formulations is the additional group velocity from the transformation $d \omega=V d k$. For these four ad-hoc optimization criteria we will determine the minimizer for the two test cases in the next section. 


\subsubsection{Kinetic energy optimization criteria}

The exact kinetic energy for linear equations with dispersion relation $\omega=\Omega(k)$ can be written like

$$
K=\frac{1}{4 g \pi} \int|\Omega \hat{\phi}|^{2} d k .
$$

A basic ingredient of the VBM is that the kinetic energy (4I) is minimized for all fluid potentials $\Phi$ that satisfy $\Phi=\phi$ at the surface. We will look for the restricted minimization on the set of potentials given by (42) with $F$ given by (43) where we minimize with respect to the parameter $\kappa$. To make this operational for the case of a signalling problem, we have to translate the uni-directional influx of a given initial signal $\eta_{0}(t)$ to the corresponding kinetic energy. This is achieved in two steps.

First, we recall the dynamic equation $\partial_{t} \phi=-g \eta$; besides that, we realize that a uni-directional influx will lead to an initial evolution given by $\partial_{t} \phi=-i \Omega \phi$. Combining these two expressions, we get for the spatial Fourier transform of the initial surface potential $\widehat{\phi_{0}}$ :

$$
i \Omega(k) \widehat{\phi_{0}}(k)=g \widehat{\eta_{0}}(k)
$$

with $\hat{\eta_{0}}$ the spatial Fourier transform of the initial profile $\eta_{0}(x)$. The kinetic energy now becomes

$$
K=\frac{g}{4 \pi} \int\left|\hat{\eta}_{0}(k)\right|^{2} d k .
$$

In a second step we relate the spatial Fourier transform of $\eta$ to the temporal Fourier transformation $\breve{\eta}_{0}(\omega)$ of the wave elevation $\eta_{0}(t)$ at $x=0$. Realizing that for uni-directional propagation it holds that

$$
\eta(x, t)=\int \widehat{\eta}_{0}(k) e^{i(k x-\Omega(k) t)} d k=\int \breve{\eta}_{0}(\omega) e^{i(K(\omega) x-\omega t)} d \omega,
$$

and that $d \omega=V(k) d k$, we get from $\breve{\eta}_{0}(\omega) d \omega=\widehat{\eta}_{0}(k) d k$ that

$$
\breve{\eta}_{0}(\omega) V(K(\omega))=\widehat{\eta_{0}}(K(\omega)) .
$$

Substituted in the last expression for the kinetic energy we get

$$
K=\frac{g}{4 \pi} \int\left|\breve{\eta}_{0}(\omega)\right|^{2} V(\omega) d \omega=\frac{g}{2} \int S(\omega) V(\omega) d \omega,
$$

where we simply write $V(\omega)=V(K(\omega))$ and $S(\omega)=\frac{\breve{\eta}_{0}(\omega) \cdot \breve{\eta}_{0} *(\omega)}{2 \pi}$ for the power spectrum.

In the case of the VBM the dispersion relation depends on $\kappa, \Omega=\Omega_{\kappa}$, and correspondingly $V(\omega)=V_{v}(\omega)$ with $v=\Omega_{\mathcal{K}}(\kappa)$. Hence for a given power spectrum the minimization problem becomes 


$$
K_{v}=\frac{g}{2} \int S(\omega) V_{v}(\omega) d \omega \rightarrow \min _{v}
$$

This is the minimization problem we will consider as the 'natural' way to find the optimal parameter $v$ and the related $\kappa$.

Observe that for the exact dispersion relation we would obtain the lowest minimal value, so that (58) provides the same optimal value as

$$
K_{v}-K_{e x}=\frac{g}{2} \int S(\omega)\left[V_{v}(\omega)-V_{e x}(\omega)\right] d \omega \rightarrow \min _{v}
$$

In Section 3.5 we will conclude that this optimization criterion leads to acceptable results for the two test cases to be considered.

\section{$3 \cdot 4$ TEST CASES}

We consider two cases of focussing wave groups. We describe in this section the main characteristics of the initial signal and spectrum, consider the evolution with the accurate $\mathrm{AB}$-equation for non-linear and linear evolutions and compare these with the measurement at the focussing point. We will use these numerical simulations in Section 3.5 to be able to quantify the quality of the optimized VBM-model.

\subsubsection{Focussing wave groups}

Both cases are examples of constructed waves that were designed for use at MARIN, the Maritime Hydrodynamic Laboratory Netherlands, to generate high waves in a long wave tank. The design is to exploit dispersive focussing: short period, small amplitude waves are generated at a waveflap, followed by successively larger and longer period waves. The design is such that the longer, i.e. faster, waves catch up with the slower shorter waves at a predetermined position in the tank. This dispersive focussing requires a broad spectrum, and the different speeds of the different frequency components determine the focussing process in a critical way. Hence, the behaviour will be most sensitive for perturbation in the phase speeds, which is why we choose these examples.

For these cases real laboratory measurements in a wavetank with depth of $1 \mathrm{~m}$ are available: the time signal at a waveflap $x=X_{0}=$ $0 \mathrm{~m}$ and near the focussing point at $x=X_{1}=20.8 \mathrm{~m}$ for one case (MARIN test case \#109001). And for another case (MARIN test case \#101013) the surface elevation at $x=X_{0}^{\prime}=10 \mathrm{~m}$ from the waveflap and at the (designed) focussing point $x=50 \mathrm{~m}$. The first case will be called the Mildly Focussing Group (mFG) in the following, since the maximal waveheight at the focussing position is rather mild. The other case, the Strong Focussing Group (sFG), is more extreme: just 

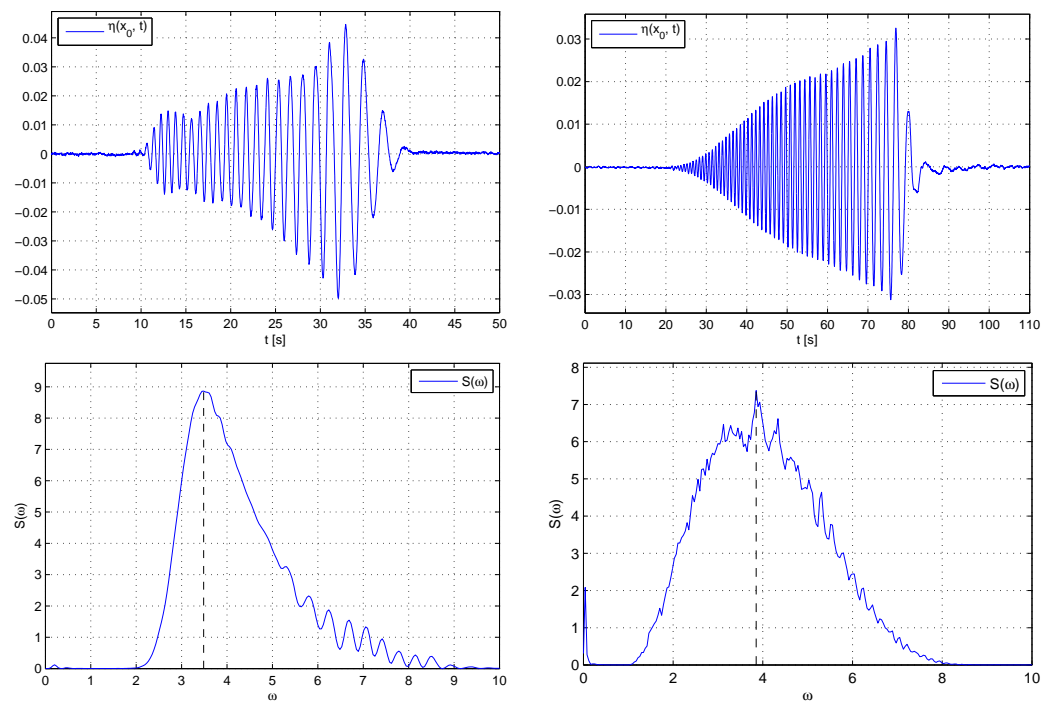

Figure 10: Focussing wave group signals and their temporal power spectra at $X_{0}=0, \mathrm{mFG}$ at the left and sFG at the right. Both signals have quite broad spectra, but sFG is more extreme than $\mathrm{mFG}$; for sFG just downstream of the focussing point some breaking (white capping) was observed.

downstream of the focussing point some breaking (white capping) was observed. For the latter case of sFG we shift in our numerical model the starting position to the position $x=X_{0}=0 \mathrm{~m}$ and accordingly the measurement position to $x=X_{1}=40 \mathrm{~m}$.

In Fig. 10, we present for each case the time signal at $X_{0}$ together with the power spectrum. Observe that the spectra (which were used in the preceding Fig. 8), are rather broad, and that sFG contains many more waves.

\subsubsection{Accurate simulation of the focussing process}

The MARIN measurement of the elevation at $X_{1}$ is the only available information downstream of $X_{0}$. This gives only little information, which is why we performed additional numerical calculations. Another reason is that the experimental data include nonlinear effects, while we are here especially interested in the linear dispersive properties.

Therefore we performed calculations with a very accurate and efficient model for uni-directional waves, i.e. the $\mathrm{AB}$-equation derived by Van Groesen \& Andonowati ([2], see [3] for numerical results). The results with the linear version of this code - which uses the exact dispersion - will be used to compare with VBM calculations in the next section for various $v$-values. The remainder of this section is to justify 

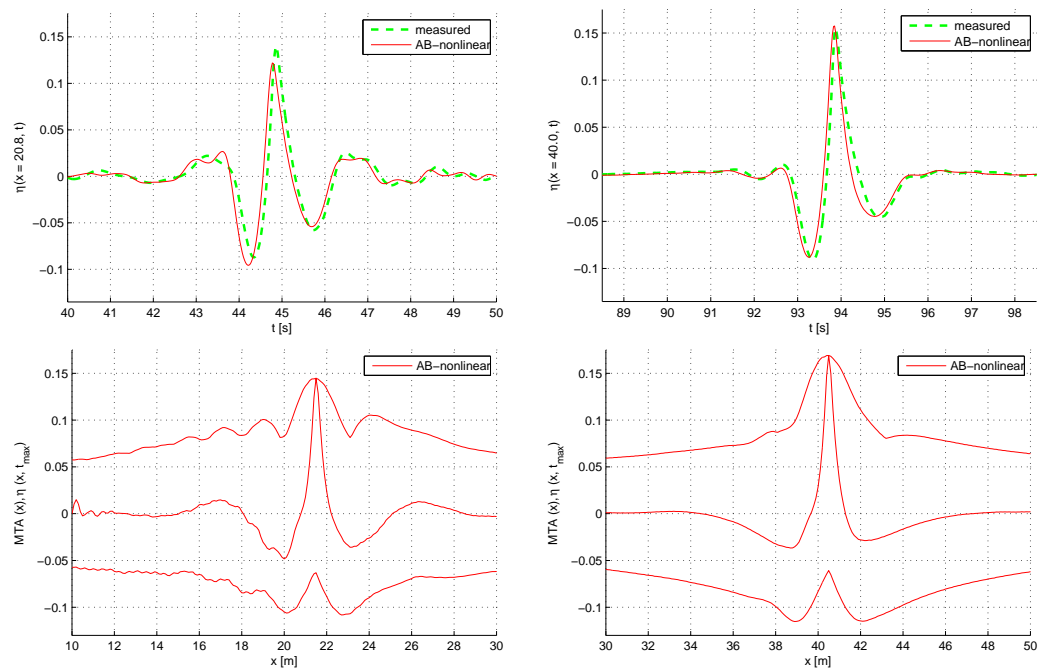

Figure 11: At the left for $\mathrm{mFG}$ and at the right for sFG are shown in the upper pane the time signals at the focussing point (respectively $X_{1}=20.8$ and $40 \mathrm{~m}$ ) of the measurement and of the nonlinear $\mathrm{AB}$ simulation. In the lower pane $\mathrm{MCH}, \mathrm{MTD}$ and the maximal wave elevation are shown as calculated by the nonlinear AB-equation.

that we can take the linear AB-simulations as sufficiently accurate results to be valid as 'exact' results for the comparison with the VBM calculations.

First, we show the result of a nonlinear evolution with a pseudospectral implementation of the AB-equation, downstream from the measured elevation signal at $X_{0}$.

In Fig. II we show at $X_{1}$ the calculated signals and the measured signal. These results show that the simulations are remarkably accurate, even for the extreme case of sFG. In measurements and simulations the spectrum changes during the evolution due to nonlinear effects. In fact, detailed analysis of the simulations show that especially for sFG, long- and short-wave generation takes place very close to the focussing point. Since this chapter deals with the linear VBM, we also consider linear evolutions of the initial signals which is simply the evolution according to the exact dispersion, and can be done with the linearized AB-equation.

Remarkably, also for these linear simulations the wave signals at $X_{1}$ are quite similar to measurements, albeit the amplitude is somewhat less, despite the fact that nonlinear effects do play a role in these test cases.

The numerical simulations - different from the available measurements - also provide information at any point in between $X_{0}$ and $X_{1}$. To get condensed information of that evolution, we will consider 

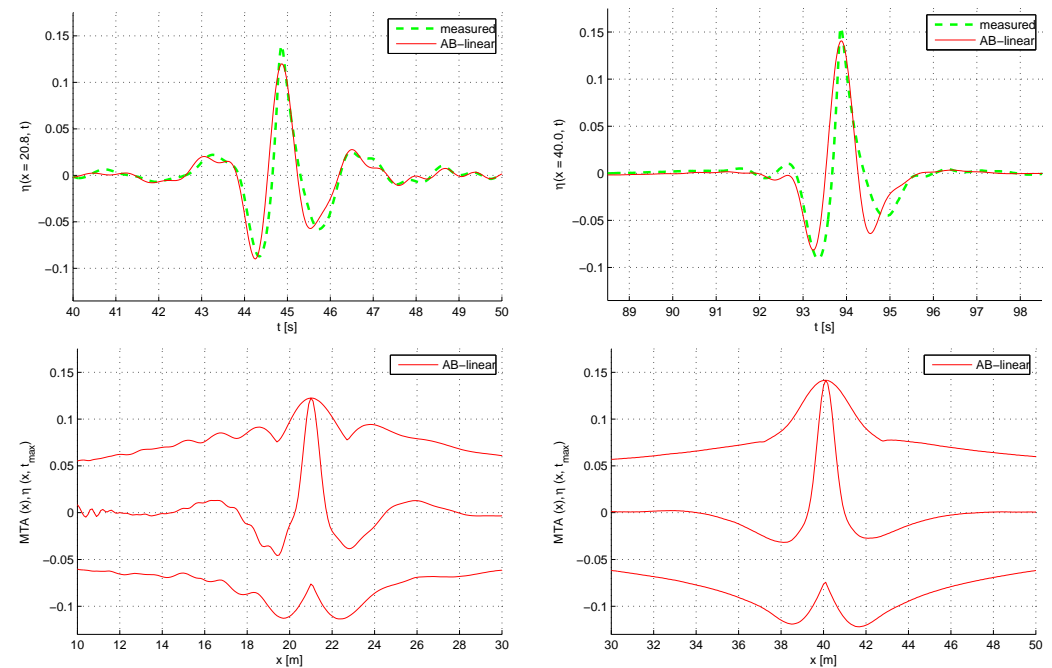

Figure 12: Similar to Fig. 11, now the AB-linear model is used. For these cases of focussing wave groups we observe that the linear simulations give results quite close to the real measurements.

the maximal temporal amplitudes: the Maximal Crest Height $(\mathrm{MCH})$ and the Minimal Trough Depth (MTD) at each position; the results are shown in Fig. 11 and Fig. 12, and we will use these to compare the MTA calculated with VBM in the next section.

\section{$3 \cdot 5$ OPTIMIZED VBM SIMULATIONS}

In this section we will first determine the optimal parameter values according to the five optimization methods discussed in Section 3.3 for both focussing wave groups. We performed numerical simulations using these optimal values in a Finite Element implementation of VBM. In Subsection 3.5.2 we present the results of the VBM calculated time signals at the focussing points for the optimal values. In Section 3.5.3 we provide the results when looking at the downstream evolution as measured by the MTA's. For both test cases the kinetic energy (KE) optimized value performed best.

\subsubsection{Calculation of optimal values}

Using the initial power spectra of the two wave groups we show in Fig. 13 the plots of the four least square errors defined in Subsection 3.3.1 and the values of the kinetic energy error as a function of the parameter $v$. The lowest point of each of these curves provides the optimal value for the corresponding optimization criterion. These optimal values are 

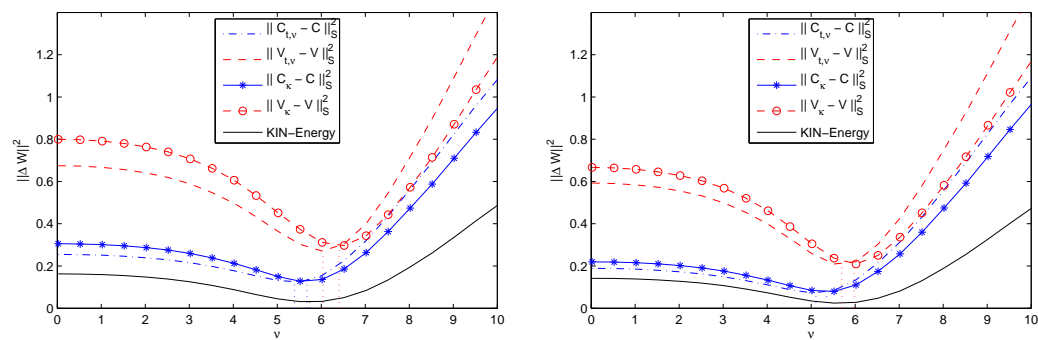

Figure 13: At the left for $\mathrm{mFG}$ and at the right for $\mathrm{sFG}$ are shown plots of the errors as functions of $v$ for the four functionals of Subsection 3.3.I and one of Subsection 3.3.2. The $v$-value at the lowest point of each curve provides the optimal value for that optimization criterion.

assembled in Table 1 . Observe that the KE-optimal value for mFG ( $v=$ 5.73 ) is best approximated by the ad-hoc optimization for spatial phase speed norm $(v=5.68)$, while for sFG the KE-optimal value $(v=5.65)$ is closest to the temporal group speed norm $(v=5.70)$.

\begin{tabular}{cccc} 
W & weight & \multicolumn{2}{c}{$v(\kappa)$ optimal } \\
& & $\mathrm{mFG}$ & $\mathrm{sFG}$ \\
\hline $\mathrm{K}$ & & $5.73(3.36)$ & $5.65(3.26)$ \\
\hline $\mathrm{C}$ & $S(\omega) d \omega$ & $5.39(2.98)$ & $5.11(2.69)$ \\
$\mathrm{C}$ & $S(k) d k$ & $5.68(3.30)$ & $5.36(2.94)$ \\
$\mathrm{V}$ & $S(\omega) d \omega$ & $6.04(3.73)$ & $5.70(3.32)$ \\
$\mathrm{V}$ & $S(k) d k$ & $6.40(4.17)$ & $5.98(3.65)$
\end{tabular}

Table 1: The first row with numerical values provides for $\mathrm{mFG}$ and $\mathrm{sFG}$ the optimal values $v$ (and $\kappa$ in parentheses) according to the kinetic energy optimization. The next rows give the optimal values for for the four ad-hoc error-minimization norms of Subsection 3.3.1

\subsubsection{Signal at focussing point}

We used a Finite Element implementation of the optimized VBM to calculate the time signal at the focussing point, with the optimal values obtained from the kinetic energy optimization, as given in Table 1 . The result is shown for both test cases in Fig. 14, upper row. To compare the results, we also depicted in the same plot the signal as calculated with the linear exact dispersion code mentioned in Section 3.4. It can be observed that the main wave is relatively well represented for both 

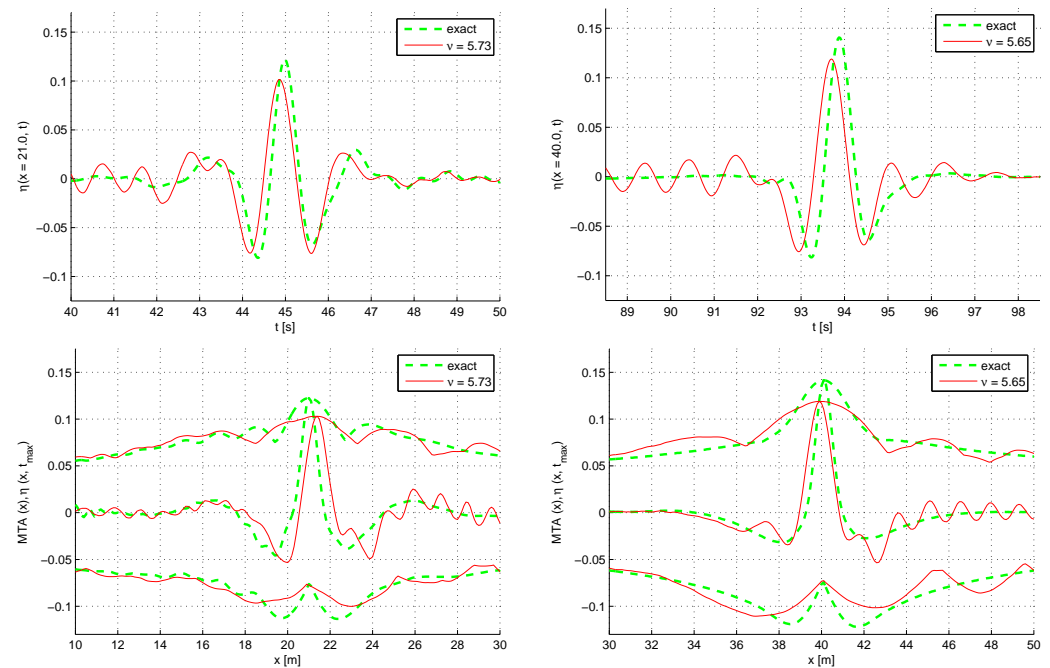

Figure 14: The mFG test case is shown to the left, the sFG test case at the right. Above are the temporal signals at the focussing point $X_{1}$, and below are the $\mathrm{MCH}, \mathrm{MTD}$ and maximal amplitude wave elevations, simulated with the VBM-code for the optimal $v$-value according to the KE-optimization, and compared to the exact dispersive linear simulation at the focussing time.

cases, but that certain frequency components disturb the signal before and after the time of focussing.

In order to compare the sensitivity of the result for any value of $v$, we calculated for each $v$ in the interval from $v=0$ till $v=10$ the signal error at the position $X_{1}$

$$
\Delta S_{1}(t)=\left|\eta_{v}\left(X_{1}, t\right)-\eta_{e x}\left(X_{1}, t\right)\right|,
$$

and the $L_{2}$-norm of the difference of the VBM signal with the 'exact' signal, over the time interval that includes the dispersive focussing and defocusssing, i.e. from $t_{0}=40 \mathrm{~s}$ till $t_{1}=50 \mathrm{~s}$ for $\mathrm{mFG}$ and from $t_{0}=89$ $\mathrm{s}$ till $t_{1}=99 \mathrm{~s}$ for $\mathrm{sFG}$ :

$$
\left\|\Delta S_{1}\right\|^{2}=\int_{t_{0}}^{t_{1}}\left[\eta_{v}\left(X_{1}, t\right)-\eta_{e x}\left(X_{1}, t\right)\right]^{2} d t .
$$

The results are shown in Fig. 15 and Fig. 16; it should be remarked that enlarging the integration span does not affect much the calculated results. The minimal value of this curve is at $v=5.09$ and $v=4.97$ for $\mathrm{mFG}$ and $\mathrm{sFG}$ respectively. Referring to Table 1 , these values are somewhat smaller than the optimal $v$-value, obtained from the KEoptimization criterion. The use of this norm to measure the error is, however, somewhat dubious, since a small phase error, as is clearly visible in Fig. 14, contributes largely to this error. 

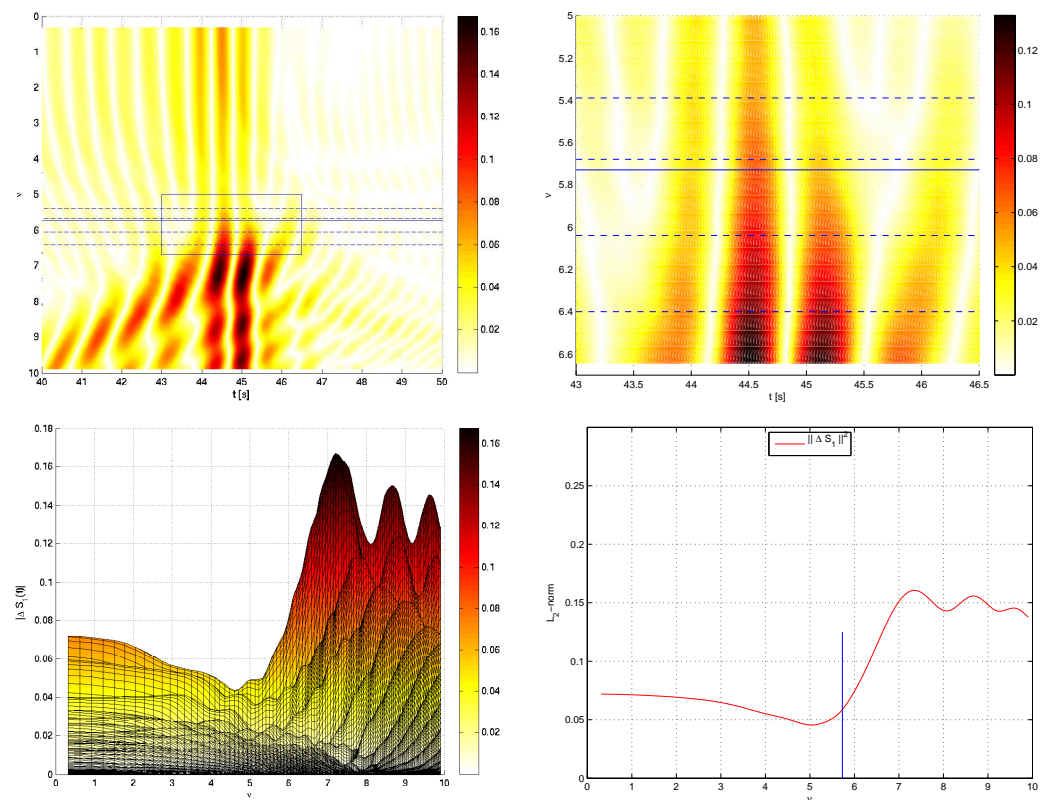

Figure 15: Density plots of the signal error (60) at $X_{1}$ for the mFG test case are shown in the top pane (at the right a zoom-in): the difference between signals of the exact dispersive simulations and VBMhyperbolic simulations, as functions of $v, t$. The solid horizontal line correspond to the the KE-optimal $v$-value, the dashed lines show the ad-hoc optimal $v$-values. A side view of the surface is shown below at the left and the $L_{2}$-error (61) is shown below at the right. 

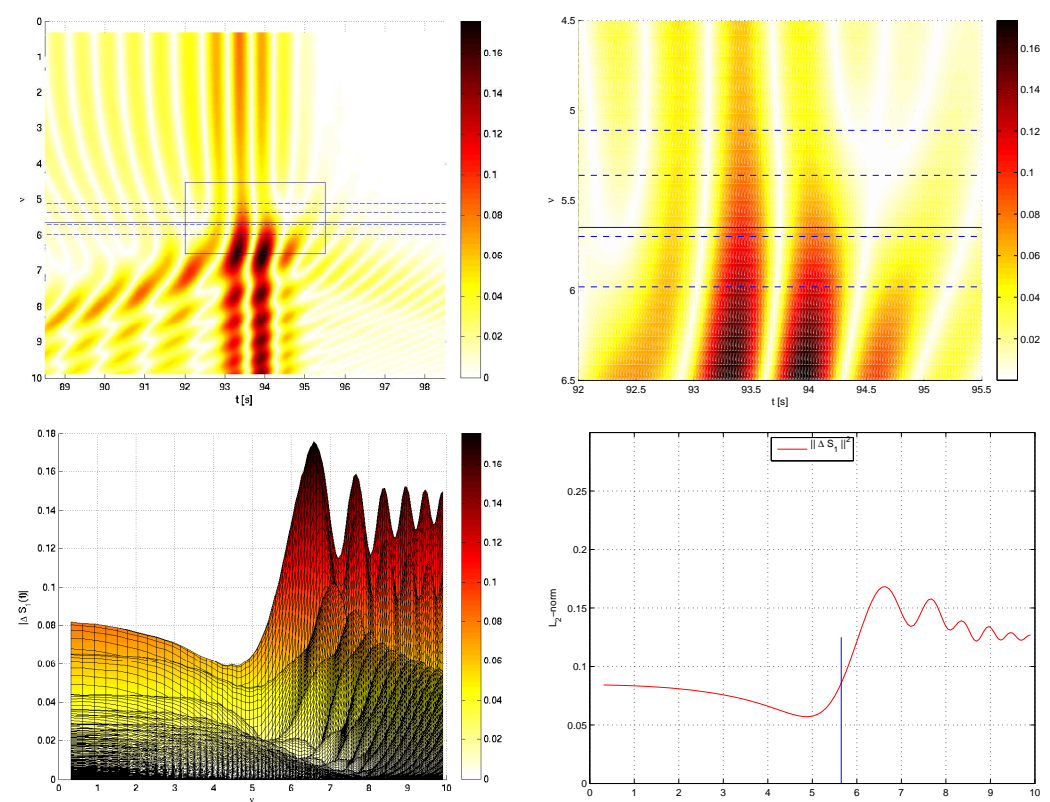

Figure 16: Similar to Fig. 15, but now for the sFG test case.

\subsubsection{Maximal wave heights comparisons}

In Fig. 14, second row, we show the spatial wave profile at the time of focussing as calculated with the KE-optimal VBM and for comparison, the 'exact' fully dispersive wave profile. Understandably, the additional oscillations in the VBM time signal in the first row, also have effects on this spatial profile. In the spatial plots we also show the curves of Maximal Crest Height $(\mathrm{MCH})$ and Minimal Trough Depth (MTD) for each simulation. These maximal temporal amplitudes give a condensed indication of the downstream running wave process.

Therefore, we considered the difference of the exact calculation with the VBM simulations for all values of the parameter $v$. The results are shown in Fig. 17 for $\mathrm{mFG}$ and in Fig. 18 for sFG. The density plots do not give much interpretable information, but the plots on the lowest row provide a precise value for $v$ for which the maximal error over the whole running down area is as small as possible. These values are given in Table 2 along with other optimal values of the parameter $v$ (or corresponding $\kappa$ ), calculated as minima of $L_{2}$-norms of the appropriate errors. The integration for the MTA's differences is done in the symmetric interval around $X_{1}$ : for $\operatorname{mFG} x \in[11,31]$ and for SFG $x \in[30,50]$; enlarging the integration span does not affect much the results. 


\begin{tabular}{ccc} 
difference & \multicolumn{2}{c}{ optimal $v(\kappa)$} \\
& $\mathrm{mFG}$ & $\mathrm{sFG}$ \\
\hline $\max _{x} \Delta M C H_{v}(x)$ & $5.90(3.55)$ & $5.82(3.46)$ \\
$\max _{x} \Delta M T D_{v}(x)$ & $6.18(3.89)$ & $5.63(3.24)$ \\
$\left\|\Delta M C H_{v}(x)\right\|$ & $5.83(3.47)$ & $5.41(3.00)$ \\
$\left\|\Delta M T D_{v}(x)\right\|$ & $5.81(3.45)$ & $5.42(3.01)$ \\
$\left\|\Delta S_{1}\right\|$ & $5.02(2.60)$ & $4.87(2.45)$ \\
\hline KE-optimization & $5.73(3.36)$ & $5.65(3.26)$
\end{tabular}

Table 2: Optimal values for mFG and sFG cases, calculated according to the difference between exact dispersion and VBM simulations. The first two rows show the values $v$ for which the maximal error over the whole running area is as small as possible. The next two rows represent $L_{2}$ errors of the difference of the $\mathrm{MCH}$ and MTD. Then the $L_{2}$-error of a signal is provided (see the previous subsection), and the last row shows the optimal values according to the kinetic energy optimization.

We see that the best value for $\mathrm{MCH}$ for $\mathrm{mFG}$ is given by $v=5.83$, which is close to the KE-optimum $v=5.73$; the best value for MTDerror is almost the same as this KE-optimum as well. For sFG the best values of $v$ are now more pronounced, given by $v=5.41$ for $\mathrm{MCH}$ and by $v=5.42$ for MTD, both quite close to the KE-optimum value.

In Fig. 19 we plot the curves of the $L_{2}$-norm of the MTA-differences. The same figure presents the plots of the maximal crest height 'positioning error', i.e. $\left|X_{v}^{\max }-X_{e x}^{\max }\right|$, where these $x$-values correspond to places in a domain, where the wave of maximal amplitude is obtained; similar for the minimal trough depth. Observe that the proposed KEoptimal choice of the parameter $v$, the first in Table 1 , is very close to the errors' minima.

\subsection{CONCLUSIONS AND REMARKS}

The freedom in the Variational Boussinesq Model (VBM) to choose the vertical profile of the fluid potential was exploited in this chapter by determining the optimal parameter value in a parameterized class of profiles. This parameter is an effective wave number, the potential profile of which is taken as an approximation of the potential profile of all other waves with different wave numbers. The optimal parameter was found from an interesting minimum kinetic energy principle that depends on properties of the influxed signal (or of an initial wave profile). Hence, in contrast with most other wave models, optimal dispersive 

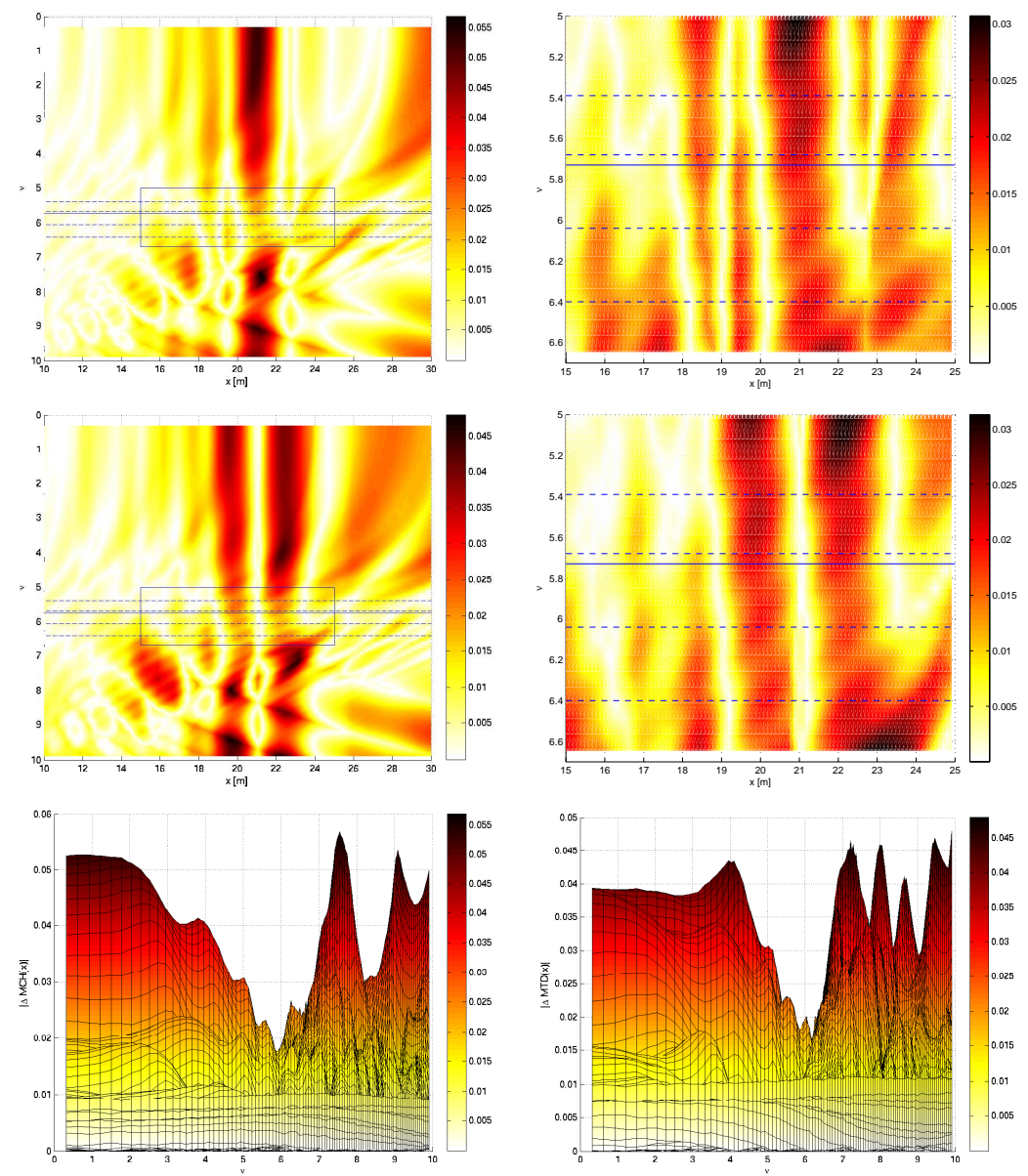

Figure 17: Density plots of $\Delta-\mathrm{MCH}$ (first row) and $\Delta$-MTD (second row) for the mFG case: the differences between the exact dispersive simulations and VBM-hyperbolic simulations, as functions of $v, x$. The solid horizontal line correspond to the KE-optimal $v$-value, the dashed lines show the ad-hoc optimal $v$-values. Two side views of the surfaces are shown in the third row: $\triangle-\mathrm{MCH}$ to the left and $\Delta$-MTD to the right. 

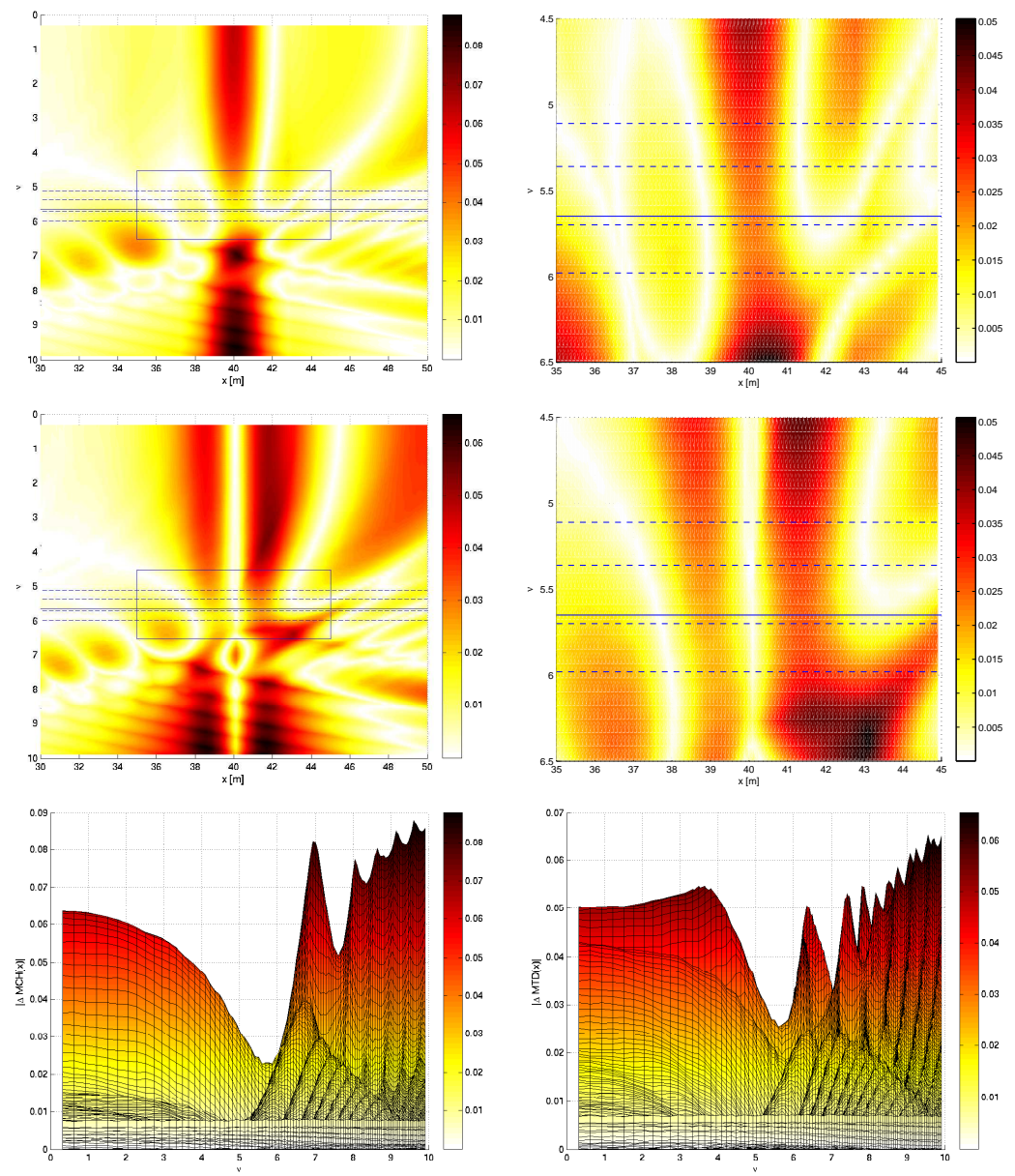

Figure 18: Similar to Fig. 17, but for the sFG test case. 

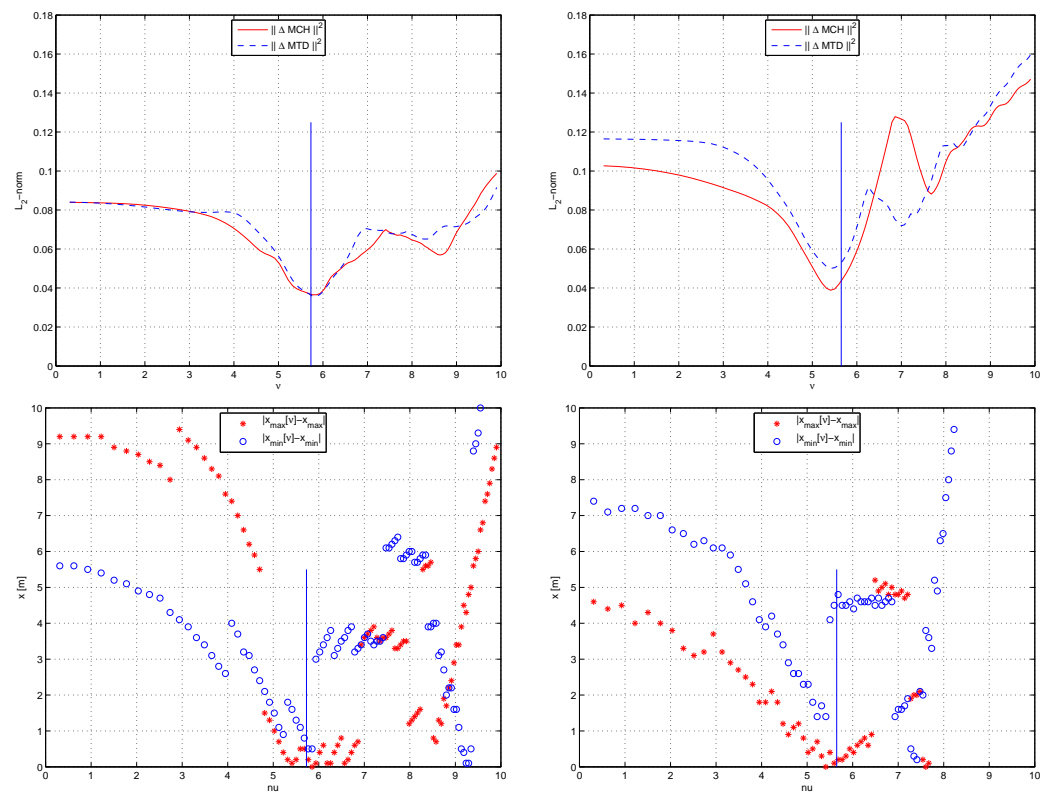

Figure 19: Calculated errors for $\mathrm{mFG}$ at the left and $\mathrm{sFG}$ at the right. The difference is given between the VBM-hyperbolic simulations with varying parameter $v$ and the exact dispersive code. The $L_{2}$-errors $\left\|M T A_{v}-M T A_{e x}\right\|$ are shown above, and the maximum's positioning errors $\left|X_{v}^{\max }-X_{e x}^{\max }\right|$ are shown below. The solid vertical line correspond to the the KE-optimal $v$-value. 
properties are determined before the model is used for simulating the evolution.

The quality of this optimal dispersion can be seen in Fig. 9 from the errors in phase and group velocity at all frequencies of the spectra. A good simulation of the focusing process requires all participating waves to evolve accurately. The result of the optimal performance is shown in Fig. 14. In both considered cases the spatial and temporal positioning of the maximal (focused) wave is rather accurate. The amplitudes of the maximal waves are too small, around $20 \%$ and $15 \%$ for the mild and strong case respectively, but the wave shape is well simulated. Some additional oscillations are noticeable which result from errors in the higher frequencies.

To make such visual observations more quantitative, we showed for both cases that the optimal parameter choice is close to optimal values, where error measures are minimal. The measures we used to judge the quality of the simulation are pointwise and integrated errors over long spatial and temporal intervals that include the essential deformations before and after focusing. The combined results for these different errors give support to the conclusion that the optimal wave number from the kinetic energy principle produces good results. This implies that if better approximations are desired, the choice of the parameterized family, provided here by (42) and (43), has to be improved, for instance by taking a superposition of various (parameterized) profiles. This opens up new opportunities for extended optimized VBMs, where the optimization should use the kinetic energy principle introduced here. This will be the topic of the next chapter. 

[1] L.J.F. Broer, On the Hamiltonian theory of surface waves, Appl. Sci. Res. 30 (1974) 340.

[2] E. van Groesen, Andonowati, Variational derivation of KdV-type of models for surface water waves, Phys. Lett. A 366 (2007) 195201.

[3] E. van Groesen, Andonowati, L. She Liam, I. Lakhturov, Accurate modelling of uni-directional surface waves, J. Comput. Appl. Math., In Press.

[4] E. van Groesen, Jaap Molenaar, Continuum Modeling in the Physical Sciences, SIAM, Mathematical Modeling and Computation Vol. 13, 2007, 236 pp.; ISBN-13: 978-0-898716-25-2.

[5] G. Klopman, M. W. Dingemans, B. van Groesen, A variational model for fully non-linear water waves of Boussinesq type, in: Proceedings IWWWFB, 2005.

[6] G. Klopman, M. W. Dingemans, B. van Groesen, Propagation of wave groups over bathymetry using a variational Boussinesq model, in: Proceedings IWWWFB, 2007.

[7] G. Klopman, M. W. Dingemans, Reflection in variational models for linear waves, Wave Motion 47 (2010) 469-489

[8] I. Lakhturov, E. van Groesen, Optimized Variational Boussinesq Modelling; part 1: Broad-band waves over flat bottom, Internal report, University of Twente, The Netherlands (2010). See http:// eprints. eemcs . utwente. nl/.

[9] J.C. Luke, A variational principle for a fluid with a free surface, J. Fluid Mech. 27 (1967) 395-397.

[10] P.A. Madsen, R. Murray, O. R. Sørensen, A new form of the Boussinesq equations with improved linear dispersion characteristics, Coast. Eng. 15 (1991), 371-388.

[11] P.A. Madsen, R. Murray, O. R. Sørensen, A new form of the Boussinesq equations with improved linear dispersion characteristics. Part 2. A slowly-varying bathymetry, Coast. Eng. 18 (1992), 371388.

[12] J.W. Miles, On Hamilton's principle for surface waves, J. Fluid Mech. 83 (1977) 153-158. 
[13] V.E. Zakharov, Stability of periodic waves of finite amplitude on the surface of a deep fluid, J. Appl. Mech. Tech. Phys. 2 (1968) 190194. 
When the wave spectrum is very broad, even the performance of the optimized cosh VBM with one vertical profile can become too poor. This is not a rare case: for example, for waves having the realistic JONSWAP spectrum the wave length in the spectral tail can become several times smaller than in the spectral peak (see also Section 7.3). As was shown in the previous chapter, such short waves can become important for the appropriate focussing of a wave train, adding up to $15-20 \%$ of the wave amplitude.

In this chapter we introduce the VBM with multiple profiles, and demonstrate that it can achieve excellent linear dispersion. We obtained good results for realistic test cases of focussing wave groups with broad spectra, compared to data from real-life experiments at MARIN hydrodynamic laboratory. We show results of comparing VBM calculations with one and two optimized vertical profiles with experimental data and simulations with other codes.

This chapter comprise the contents of the published article [24].

\subsection{INTRODUCTION}

In the modeling of surface water waves, Boussinesq-type equations have the advantage that they reduce the dimensionality of the flow problem by modelling the effects of the interior flow into a simplified description with variables in the horizontal plane, the elevation and a velocity (or potential) type of quantity. Starting from the original paper by Boussinesq [5], the work in this field concentrated at improving dispersive and nonlinear properties of the models, extending them to two (horizontal) dimensions, variable bathymetry, etc. The development obtained a boost in recent decades for the aim to get reliable numerical implementations; see $[9,17,18,31,32]$ for reviews.

The most common ways to derive such Boussinesq equations are small parameter expansion techniques in the full set of water equations, using the inverse wavelength and the amplitude as expansion parameters. The approximation of the interior flow determines the dispersion properties of the model. Dispersive properties of Boussinesqtype systems were studied and improved in many articles. Quite common is to incorporate Padé-approximants of different orders into the dispersion relation of a model. For example, Madsen et al. [28, 29] and Nwogu [35] demonstrate Padé $(2,2)$ dispersion characteristics. Schäffer \& Madsen [39] obtained dispersion accurate up to $k h \approx 6$; Mad- 
sen \& Schäffer [30] and Zou [45] used Padé approximation $(4,4)$ and Lynett \& Liu [27] Padé $(2 N, 2 N)$.

A somewhat different way to derive Boussinesq equations is to use the variational structure of the surface water problem. Luke [26] formulated a pressure principle for the complete water problem. This variational principle can be transformed to an action principle see e.g. [11], leading to a set of Hamilton equations that describe the dynamics directly in surface variables as canonical variables (elevation and fluid potential). The Hamilton equations were discovered (in a different way) by Zakharov [42]; see [37, 38, 43] for reviews. Although the basic abstract formulation does not need any approximation, to become applicable the kinetic energy functional in the Hamiltonian has to be expressed explicitly in the surface variables. And this requires an approximation of the Laplace problem for the interior flow. The difference with the expansion methods mentioned above is that by approximating the Hamiltonian, the approximate equations retain the Hamiltonian character, with conservation properties as consequence: energy conservation from time invariance and momentum conservation from translation invariance above flat bottom. This methodology has been followed in various ways. One way is to approximate the Dirichlet-to-Neumann (DtN) operator that appears in the kinetic energy, as done by Craig \& Sulem [8], see also [3, 41], using a convergent Taylor approximation of the DtN operator.

Different from this, Van Groesen \& Andonowati [12] used the surface elevation and the potential at the still water level as primary variables. The Hamiltonian structure is retained by performing this (noncanonical) transformation in Luke's action principle. Then the DtN operator is explicit, and approximations of the Hamiltonian were derived using Taylor expansions around the still water level for which the Hamiltonian is exactly known when the bottom is flat. Using spectral methods, the pseudo-differential operators can be handled as multiplications in Fourier space, and dispersive properties are exact for infinitesimal waves of any wavelength. For varying bottom, the quasihomogeneous approach is applied to operators in the Hamiltonian, which leads to consistent symmetries for operators in the resulting Hamiltonian equations, which would not be the case if this procedure is applied directly in the Hamiltonian equations. In $[14,15]$ the $A B$ equations from [12] and generalizations were described, together with hybrid spatial-spectral numerical implementations.

The Variational Boussinesq Model (VBM) also uses the Hamiltonian formulation. But now the kinetic energy is approximated, as a variant of the Green-Naghdi method [10], see Klopman e.a. [19, 22]. The internal fluid velocity is approximated as a combination of a priori chosen vertical profiles with coefficients that depend on the horizontal directions. These coefficients become independent variables that have 
to satisfy a system of elliptic equations that has to be solved together with the dynamic equations for the surface elevation and the fluid potential at the surface. The main advantages of this method are properties of practical importance for a numerical implementation: the VBM Hamiltonian is positive-definite, the resulting PDE system contains no mixed space-time derivatives and the order of the derivatives is two or less. The last property is helpful for Finite Element and FD discretizations. The VBM approach can be easily formulated for one and two horizontal dimensions and for flat and variable bottom.

The applications in [22] can all be characterized by the fact that only one vertical profile is enough to obtain sufficiently good results for the cases treated there. With either a parabolic profile or one profile as in Airy's linear theory, a restricted interval of wavenumbers can be well approximated. But for wave fields with a broad spectrum, such as irregular waves or focussing wave groups, it turns out that the dispersive properties cannot be approximated sufficiently accurate on the whole interval. Therefore, in this chapter we study an extension that uses combinations of vertical profiles. In addition we extend the kinetic energy optimization criterion (58) that provides the best choice of such profiles; the optimization will depend on the spectrum of the signal at the influx position. This leads to the variant that will be called the Optimized VBM.

We show the performance of the extended optimization with a test case of a focussing wave group with a broad spectrum. This wave group has been measured at MARIN hydrodynamic laboratory, Wageningen, the Netherlands, test number 101013, on water depth $h=1$ $\mathrm{m}$, which we refer to as FWG. We compare the experimental data with calculations with Optimized VBM for the cases of one and two vertical profiles. It will turn out that the optimal vertical potential profile(s) are in all cases far away from the profile at or near the peak-wave number, which could be a first guess for an optimum. Since strong nonlinear effects appear near focussing, we also compare the calculations with the linear and nonlinear versions of the AB-equation [12, 13, 14, 15] that has exact dispersive effects in first and second order. Furthermore, comparisons will be shown with simulations using the commercial software tool MIKE 21 [33] and the new open source model SWASH [40].

The outline of the chapter is as follows. In Section 4.2 we present the dynamic equations, investigate the dispersion relation for one and more vertical profiles, and give some details about the numerical implementation. In Section 4.3 we derive the novel optimization principle to calculate the optimal (Airy) profiles. In the next Section 4.4 we describe the test case of the focussing wave group, and show results of simulations with the very accurate (nonlinear) unidirectional ABequation. In Section 4.5 we perform linear and nonlinear calculations 
with the optimal profiles according to the kinetic energy optimization criterion, and compare the Optimized VBM calculations with real-life measurements, the AB-simulations and simulations with MIKE 21 and SWASH. In Section 4.6 we provide some remarks and conclusions.

\subsection{VARIATIONAL BOUSSINESQ MODEL WITH MULTIPLE PROFILES}

\subsubsection{Hamiltonian evolution equations}

As written in the introduction, every Variational Boussinesq model is obtained by approximating the kinetic energy. We use the canonical variables to describe the fluid equations, the surface elevation $\eta$ and the fluid potential $\phi$ at the free surface. The Hamiltonian is the total energy, of which the kinetic energy is given by

$$
K(\phi, \eta)=\iint_{-h}^{\eta} \frac{1}{2}|\nabla \Phi|^{2} d z d x
$$

and the potential energy by

$$
P=\frac{1}{2} \int g \eta^{2} d x
$$

The Hamiltonian equations are given with $H=K+P$ by:

$$
\partial_{t} \eta=\delta_{\phi} H, \quad \partial_{t} \phi=-\delta_{\eta} H
$$

as follows from Luke's variational principle [11, 26] (see also [6, 34, 42]) by taking variations of the Lagrangian

$$
\delta \mathcal{L}=\delta \int\left(\int \phi \partial_{t} \eta d x-H(\phi, \eta)\right) d t=0 .
$$

Exact equations are obtained if we could assure that the fluid potential $\Phi$ satisfies the Laplace equation in the interior, the impermeability condition at the bottom $z=-h$ and the prescribed value $\Phi=\phi$ at the free surface $z=\eta$. In this chapter we consider the bottom to be flat; the case of varying bottom is dealt with in [2] and in the next chapter. This exact potential $\Phi$ has the extremal property that it minimizes the kinetic energy over all potentials that satisfy the prescribed surface value $\phi$. This minimization property of the kinetic energy will be exploited in two essential ways to obtain the best dispersive properties in the rest of this chapter.

Since the Laplace problem cannot be solved explicitly for nontrivial $\eta$, the kinetic energy has to be approximated to make the model useful for numerical simulations. 
In $[19,20,21]$ the Ansatz taken for $\Phi$ contains one vertical profile. Here, as suggested in [22], we take the approximation that follows by writing $\Phi$ as a multiple term perturbation of the surface potential:

$$
\Phi(x, z)=\phi(x)+\Sigma_{m} F_{m}(z) \psi_{m}(x)=\phi(x)+F(z) \cdot \Psi(x),
$$

where $F$ and $\Psi$ are vector functions. To satisfy the surface condition we require $F_{m}(\eta)=0$, and for the bottom impermeability condition $F_{m}^{\prime}(-h)=0$. The vertical profile functions $F_{m}$ have to be chosen in advance, and the functions $\psi_{m}$ become additional free surface variables, for which additional elliptic equations have to be solved, together with $\eta$ and $\phi$.

The dispersion relation of the resulting dynamical system depends (strongly) on the choice of the function $F_{m}$. Taking all functions equal to zero leads to the shallow water equations (SWE) with no dispersion. From Airy linear theory, the fluid potential of a small amplitude harmonic wave with wave number $\kappa$ can be exactly represented by the choice

$$
f(z ; \kappa)=\frac{\cosh \kappa(z+h)}{\cosh \kappa(\eta+h)}-1 .
$$

But for non-harmonic waves, or when nonlinearity is essential, the form (65) can at best be approximative, and multiple functions will improve the approximation as we will see.

\subsubsection{Dispersion relation}

In this subsection we address the question for which choices of $F_{m}$ one gets the best dispersive properties. We will keep the form (65) in Ansatz (64) for each function $F_{m}$; in the following we will use the notation $f_{m}=f\left(z ; \kappa_{m}\right)$ and allow the value of $\kappa_{m}$ to be chosen in an optimal way. That is, the aim will be to choose the $\kappa_{m}$-s in such a way that the difference with the exact dispersion relation, given by

$$
\omega=\Omega_{e x}(k)=\operatorname{sign}(k) \cdot \sqrt{g k \tanh k h},
$$

is as small as possible for relevant wave numbers. But it is not obvious which norm to choose for measuring the difference. Moreover, it is not simple to know what the effect is of differences in the dispersion relation on the behaviour of the wavefields.

We assume the contribution of $\partial_{x} F$ in (62), coming from the dependence of $F$ on $\eta$, to be small and neglect it (so-called weak nonlinear model). The kinetic energy is then given by

$$
K \approx \frac{1}{2} \int\left[(h+\eta)\left(\partial_{x} \phi\right)^{2}+\alpha \partial_{x} \Psi \cdot \partial_{x} \Psi+\gamma \Psi \cdot \Psi+2 \partial_{x} \phi \beta \cdot \partial_{x} \Psi\right] d x
$$


with integral coefficients that are the elements of the matrices $\alpha$ and $\gamma$ and the column vector $\beta$, which depend on the approximation of the vertical potential profiles and are given by

$$
\alpha_{j m}=\int_{-h}^{\eta} f_{j} f_{m} d z, \quad \beta_{m}=\int_{-h}^{\eta} f_{m} d z, \quad \gamma_{j m}=\int_{-h}^{\eta} f_{j}^{\prime} f_{m}^{\prime} d z .
$$

Explicit expressions are given in the Appendix. For a parabolic approximation of the profile F, taken in [19] and [20], they depend only on depth. For the cosine hyperbolic approximation, on which we concentrate in this chapter, the coefficients depend also on the wave number $\kappa$; the value of this parameter will shortly be determined in an optimal way.

Variations of the Lagrangian with respect to $\phi, \eta$ and $\psi_{m}$-s give the following linear system of PDE-s

$$
\left\{\begin{array}{l}
\partial_{t} \eta=-\partial_{x}\left((h+\eta) \partial_{x} \phi\right)-\partial_{x}\left(\beta \partial_{x} \Psi\right) \\
\partial_{t} \phi=-g \eta-\frac{1}{2} \partial_{x} \phi^{2} \\
-\partial_{x}\left(\alpha \partial_{x} \Psi\right)+\gamma \Psi=\partial_{x}\left(\beta \partial_{x} \phi\right) .
\end{array}\right.
$$

To investigate the dispersion quality of the model, we restrict to the linearized equations of (69) by replacing in (62) the vertical integration interval till the still water level 0 instead of till the surface elevation $\eta$, and putting $\eta=0$ in (65) and (68). The dispersion relation of the linearized model is now described for harmonic profiles with frequency $\omega$ and wave number $k$ by the dispersion relation $\omega=\Omega_{V B M}(k)=k C_{V B M}(k)$ with the phase velocity

$$
C_{V B M}=c_{0} \sqrt{1-\frac{k^{2}}{h} \beta \cdot\left(\gamma+\alpha k^{2}\right)^{-1} \beta,}
$$

where $c_{0}=\sqrt{g h}$ is the speed of long waves. Here we assumed that all parameters $\kappa_{m}$ are different, so that the matrix $\left(\gamma+\alpha k^{2}\right)$ is invertible.

It appears, that unlike the exact phase speed, this approximation has a nonzero limit for short waves. Particularly, for the 1-profile approximation it holds that

$$
\lim _{k \rightarrow \infty} C_{V B M}(k)=c_{0} \sqrt{1-\frac{\beta_{1}^{2}}{\alpha_{11} h}}>0 .
$$

Indeed, this limit is real and nonzero as a consequence of the fact that $\beta_{1}^{2} \leqslant \alpha_{11} h$, because of the Cauchy-Schwarz inequality:

$$
\left(\int_{-h}^{0} f_{1} \cdot 1 d z\right)^{2} \leqslant \int_{-h}^{0} f_{1}^{2} d z \cdot \int_{-h}^{0} 1 d z,
$$

while equality in this expression is only possible for trivial functions $f_{1}$. 


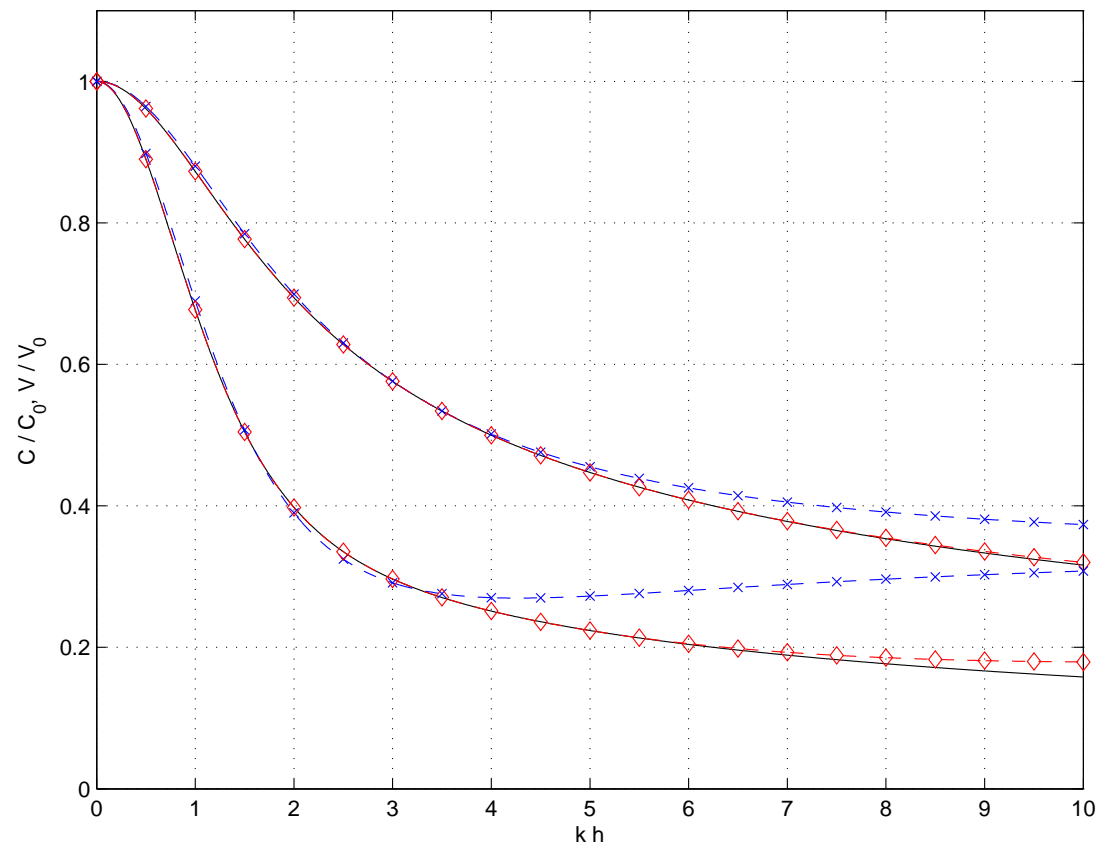

Figure 20: The phase speed (upper curves) and the group speed (lower curves) for different models: the exact dispersion (solid), the 1-profile cosine hyperbolic approximation with $\kappa_{1} h=3.27$ (dashed with crosses) and the 2-profile cosine hyperbolic approximation with $\kappa_{1} h=2.73$ and $\kappa_{2} h=5.25$ (dashed with diamonds). 
Fig. 20 shows normalized plots of the phase and group speed for the cosine hyperbolic models with 1- and 2-profiles, and of the exact phase and group speed $V_{e x}=d \Omega_{e x} / d k$.

From this plot it seems that the approximate phase velocity $C_{a p p}$ is for each wavenumber larger than or equal to the exact phase speed $C_{e x}$. This is actually true for any approximate VBM and is a direct consequence of the minimization property of the (quadratic) kinetic energy, mentioned in the introduction. Indeed, the kinetic energy can be written for any linear dispersive wave equation as

$$
K(\phi)=\frac{1}{2 g} \int|\Omega \phi|^{2} d x=\frac{1}{2 g} \int u C^{2} u d x \text { with } u=\partial_{x} \phi,
$$

where $C$ is the phase velocity. For the exact dispersion with $C_{e x}$ and an approximate VBM model, such as $C_{a p p}$ in the hyperbolic approximation, we have

$$
\begin{aligned}
K(\phi) & =\min _{\Phi=\phi \text { at } z=0} \frac{1}{2} \iint|\nabla \Phi|^{2} d z d x=\frac{1}{2 g} \int u C_{e x}^{2} u d x \\
& \leqslant \frac{1}{2} \iint\left|\nabla \Phi_{a p p}\right|^{2} d z d x=\frac{1}{2 g} \int u C_{a p p}^{2} u d x .
\end{aligned}
$$

Since this holds for each $\phi$ (each $u$ ) we conclude that $\Omega_{e x}^{2} \leqslant \Omega_{a p p}^{2}$ and $C_{e x}^{2} \leqslant C_{a p p}^{2}$. For the case of hyperbolic profiles, parametrized with wavenumbers $\kappa_{j}$, it holds that the phase speed is exact (only) for the wave numbers $k=\kappa_{j}$ :

$$
C_{e x}\left(\kappa_{j}\right)=C_{a p p}\left(\kappa_{j}\right) \text { for all } j \text {, and } C_{e x}(k)<C_{\kappa}(k) \text { for } k \neq 0, \kappa_{j} .
$$

As a consequence, we also have that the group velocity is exact at these wavenumbers $\kappa_{j}$ :

$$
V_{e x}\left(\kappa_{j}\right)=V_{a p p}\left(\kappa_{j}\right) \text { for all } j .
$$

\subsubsection{Numerical implementation}

Here we give a brief description of the numerical implementation for the VBM. For details see, e.g. $[1,2,22]$.

The VBM is obtained from a variational formulation by minimizing the Lagrangian (63) with respect to $\eta, \phi$ and $\psi_{m}$, so, it is natural to build an implementation with the Finite Element Method (FEM). One can use piecewise linear local basis functions $T_{k}(x)$ since the highest derivatives in (63) are of the first order. We discretize the solutions in space by FEM; this leads to a system of ordinary differential equations (ODEs), which we solve in time by a Runge-Kutta method.

Discretizing the variables $\eta, \phi$ and $\psi_{m}$ and varying the Lagrangian (63), we eventually arrive at a high-dimensional system of ODEs: one 
matrix equation for the vector $\underline{\eta}$, one for $\underline{\phi}$ and a coupled system of elliptic equations for $\underline{\psi_{m}}$. The matrix system is given by

$$
\left\{\begin{array}{l}
M \partial_{t} \underline{\eta}=D^{h} \underline{\phi}+\sum D^{\beta_{m}} \underline{\psi_{m}}+\underline{b} \\
M \partial_{t} \underline{\phi}=-g M \underline{\phi}-\underline{v} \\
\left(\begin{array}{ccc}
D^{\alpha_{11}}+M^{\gamma_{11}} & \ldots & D^{\alpha_{1 N}}+M^{\gamma_{1 N}} \\
\vdots & \ddots & \vdots \\
D^{\alpha_{N 1}}+M^{\gamma_{N 1}} & \ldots & D^{\alpha_{N N}}+M^{\gamma_{N N}}
\end{array}\right)\left(\begin{array}{c}
\underline{\psi_{1}} \\
\vdots \\
\underline{\psi}_{N}
\end{array}\right)=-\left(\begin{array}{c}
D^{\beta_{1}} \underline{\phi} \\
\vdots \\
D^{\beta_{N}} \underline{\phi}
\end{array}\right),
\end{array}\right.
$$

where we make use of the upper index denoting spatial distribution of weights for the so-called mass-matrix $M$ with elements $M_{i, j}^{\rho}=$ $\int \rho(x) T_{i}(x) T_{j}(x) d x$, and the so-called stiffness-matrix $D$ with elements $D_{i, j}^{\rho}=\int \rho(x) T_{i}^{\prime}(x) T_{j}^{\prime}(x) d x$; an index is omitted for the unitary weight. All the matrices here are trilinear (or just sparse if the system would be written in $2 \mathrm{D})$. The vectors $\underline{b}$ and $\underline{v}$ are nonlinear column vectors with elements $b_{j}=\sum_{i, k} \eta_{i} \phi_{k} \int T_{i}(x) T_{j}^{\prime}(x) T_{k}^{\prime}(x) d x$ and $v_{j}=\frac{1}{2} \sum_{i, k} \phi_{i} \phi_{k} \int T_{j}(x) T_{i}^{\prime}(x) T_{k}^{\prime}(x) d x$ respectively. The system is numerically solved with help of a RungeKutta method (e.g. ODE45 in MATLAB). While vectors $\eta$ and $\phi$ are obtained via the time integrator using the first two equations, the third subsystem has to be solved for $\underline{\psi}$ at each time step.

\subsection{OPTIMIZATION CRITERION}

In the first subsection we introduce the kinetic energy optimization criterion. Since this chapter is focussed on solving a signalling problem, we reformulate the expression for the kinetic energy as an integral over the power flux, and exploit the minimizing property of the kinetic energy for approximations to obtain optimal results for wave numbers of profile functions. The optimization criterion will be used in Section 4.5 to determine the quality of the resulting dynamics.

\subsubsection{Kinetic energy optimization criterion}

The kinetic energy for linear equations with dispersion relation $\omega=$ $\Omega(k)$ can be written like

$$
K(\phi)=\frac{1}{4 g \pi} \int|\Omega \hat{\phi}|^{2} d k,
$$

where $\hat{\phi}$ denotes the spatial Fourier transform of $\phi$. This thus also holds for the VBM approximation with $\Omega_{a p p}(k ; \kappa)$, and we have as above 


$$
K(\phi)=\frac{1}{4 g \pi} \int\left|\Omega_{e x} \widehat{\phi}\right|^{2} d k \leqslant \frac{1}{4 g \pi} \int\left|\Omega_{a p p}(k ; \kappa) \widehat{\phi}\right|^{2} d k \equiv K_{a p p}(\phi, \kappa) .
$$

This holds for all vectors $\kappa$.

For linear simulations with a finite number of wave lengths, we can obtain the exact evolution by the correct choice of the parameters $\kappa$. For the more realistic case that there is a continuum of wave lengths, we try to optimize the value of the vector $\kappa$, for a fixed number of parameters. Clearly, an optimal choice will depend on the characteristics of the wave field that has to be simulated. We will now show how this can be achieved for a signalling problem, but a similar (actually somewhat simpler) reasoning can be applied for an initial value problem.

For a signalling problem, a one-way travelling wave is influxed by prescribing at a certain position $x_{0}$ (which we take without loss of generality $x_{0}=0$ ) the elevation signal, to be denoted by $\eta_{0}(t)$. We will proceed in two successive steps.

First, we recall the dynamic equation $\partial_{t} \phi=-g \eta$; besides that we realize that a uni-directional influx will lead to an initial evolution given by $\partial_{t} \phi=-i \Omega \phi$. Combining these two expressions, we get for the spatial Fourier transform of an initial surface potential $\widehat{\phi_{0}}$ :

$$
i \Omega(k) \widehat{\phi_{0}}(k)=g \widehat{\eta_{0}}(k),
$$

where $\hat{\eta}_{0}$ denotes the spatial Fourier transform of the initial profile $\eta_{0}(x)$. The kinetic energy then becomes

$$
K=\frac{g}{4 \pi} \int\left|\hat{\eta}_{0}(k)\right|^{2} d k
$$

In the second step we relate the spatial Fourier transform of $\eta$ to the temporal Fourier transform $\check{\eta}_{0}(\omega)$ of the wave elevation $\eta_{0}(t)$ at $x_{0}=0$. Realizing that for uni-directional propagation it holds that

$$
\eta(x, t)=\int \widehat{\eta}_{0}(k) e^{i(k x-\Omega(k) t)} d k=\int \breve{\eta}_{0}(\omega) e^{i(K(\omega) x-\omega t)} d \omega,
$$

and that $d \omega=V(k) d k$, where $V(k)$ is the group velocity, we get from $\check{\eta}_{0}(\omega) d \omega=\hat{\eta}_{0}(k) d k$ that

$$
\check{\eta}_{0}(\omega) V(K(\omega))=\widehat{\eta_{0}}(K(\omega)) \text {. }
$$

Substituting the last expression in the expression for the kinetic energy we get

$$
K=\frac{g}{4 \pi} \int\left|\breve{\eta}_{0}(\omega)\right|^{2} V(\omega) d \omega=\frac{g}{2} \int S(\omega) V(\omega) d \omega,
$$

where we simply write $V(\omega)=V(K(\omega))$ and 


$$
S(\omega)=\frac{\check{\eta}_{0}(\omega) \cdot \check{\eta}_{0}^{*}(\omega)}{2 \pi}
$$

for the power spectrum.

In the case of the VBM with parameters $\kappa$, let $V_{a p p}(\omega, \kappa)$ be the corresponding group velocity. For the power spectrum that corresponds to the given elevation signal, the optimization problem becomes a minimization problem with respect to the wavenumbers $\kappa$ of the vertical Airy profiles of the approximate kinetic energy

$$
K_{a p p}=\frac{g}{2} \int S(\omega) V_{a p p}(\omega, \kappa) d \omega .
$$

Note that this functional, of which the density is actually the power flux, has the value of the kinetic energy, and that by equipartition, it is a constant of the motion with value half of the Hamiltonian. Observe also that the exact dispersion relation would give the lowest minimal value, so that (74) provides the same optimal value as the difference with the exact kinetic energy

$$
K_{a p p}-K_{e x}=\frac{g}{2} \int S(\omega)\left[V_{a p p}(\omega, \kappa)-V_{e x}(\omega)\right] d \omega
$$

In Section 4.5 we will show that this optimization criterion leads to acceptable results for the focussing wave group if we take one optimized vertical profile, and good results if we use two optimized vertical profiles.

\subsubsection{Profile optimization}

As stated above, for given influx signal, we choose the optimal $\kappa$ values by minimizing the expression (74). To illustrate the resulting dispersion relation for a specific case, we use the spectrum that corresponds to the influx signal that will be used to calculate the dynamic evolution of the test case FWG in the next section. For that spectrum, we show in Fig. 21 the plots of the kinetic energy error for the 2-profile case as a function of two parameters $v_{1}=\Omega\left(\kappa_{1}\right)$ and $v_{2}=\Omega\left(\kappa_{2}\right)$. The lowest points of the surface provide the optimal values $v_{1}$ and $v_{2}$ for the test case. On the diagonal, where $v_{1}=v_{2}$, we show the kinetic energy error (75) for the 1-profile case as a function of the only parameter $v_{1}$. The optimal values are shown in Table 3 , with the corresponding $\kappa$-values in parentheses.

We will now use these values to illustrate the effects on the phase and group speed of the approximate dispersion relation. 


\begin{tabular}{c|c|c} 
FWG & VBM with 1 profile & VBM with 2 profiles \\
\hline$v_{1}$ & $5.66(3.27)$ & $5.15(2.73)$ \\
\hline$v_{2}$ & & $7.18(5.25)$ \\
\hline
\end{tabular}

Table 3: For the FWG the optimal values $v_{1}$ and $v_{2}$ (and $\kappa$-s in parentheses) according to the kinetic energy optimization. To the left is the result for the 1-profile approximation, to the right for the 2-profile approximation.
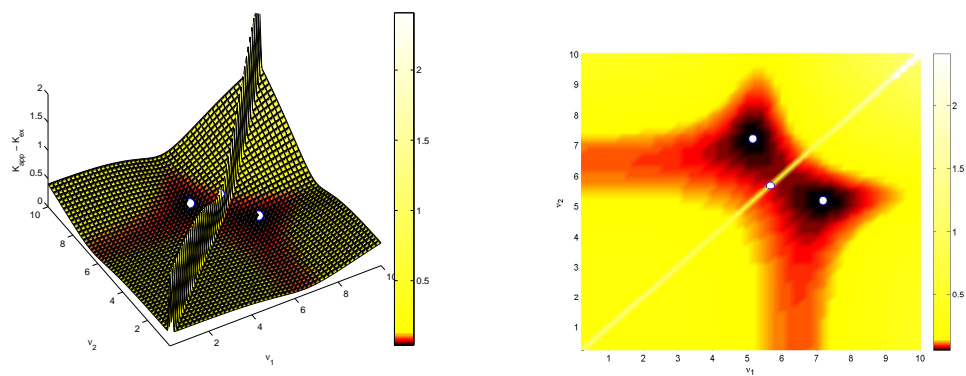

Figure 21: For the spectrum of the test case in the next section, we show at the left a side view and at the right the top view of the kinetic energy error $(75)$ as a function of $v_{1}$ and $v_{2}$. The $\left(v_{1}, v_{2}\right)$-value at the lowest point of the surface provides the optimal value of the parameters. The lowest point on the diagonal gives the optimal value $v_{1}$ for the 1-profile approximation.

In Fig. 22 is plotted the power spectrum $S(\omega)$ of the time signal of the test case, together with the exact phase speed and the phase speed error (at the top row) and the group speed with the error (at the lower row), at the left for the 1-profile optimization, at the right for 2-profile optimization.

We use the notation $C_{t}(\omega)$ with subscript $t$ to indicate that we consider the phase velocity as a function of frequency. Hence, for a given dispersion relation $\omega=\Omega(k)$, we consider the inverse $k=K(\omega)$ and define $C_{t}$ as

$$
C_{t}(\omega)=C(K(\omega))=\frac{\omega}{K(\omega)} .
$$

When using the exact dispersion relation $\omega=\Omega_{e x}(k)$, we write $C_{t, e x}$. Similarly for the group velocity $V_{t}(\omega)=V(K(\omega))$.

Note that when using the VBM-hyperbolic approximation, the transformation from wave numbers to frequencies is the same for the exact as well as for the approximate dispersion, since these coincide at $\kappa_{j}$, and so $v_{j}=\Omega_{e x}\left(\kappa_{j}\right)=\Omega_{a p p}\left(\kappa_{j}\right)$. Therefore we write $C_{t, a p p}$ to denote the VBM phase speed as function of the frequency: 

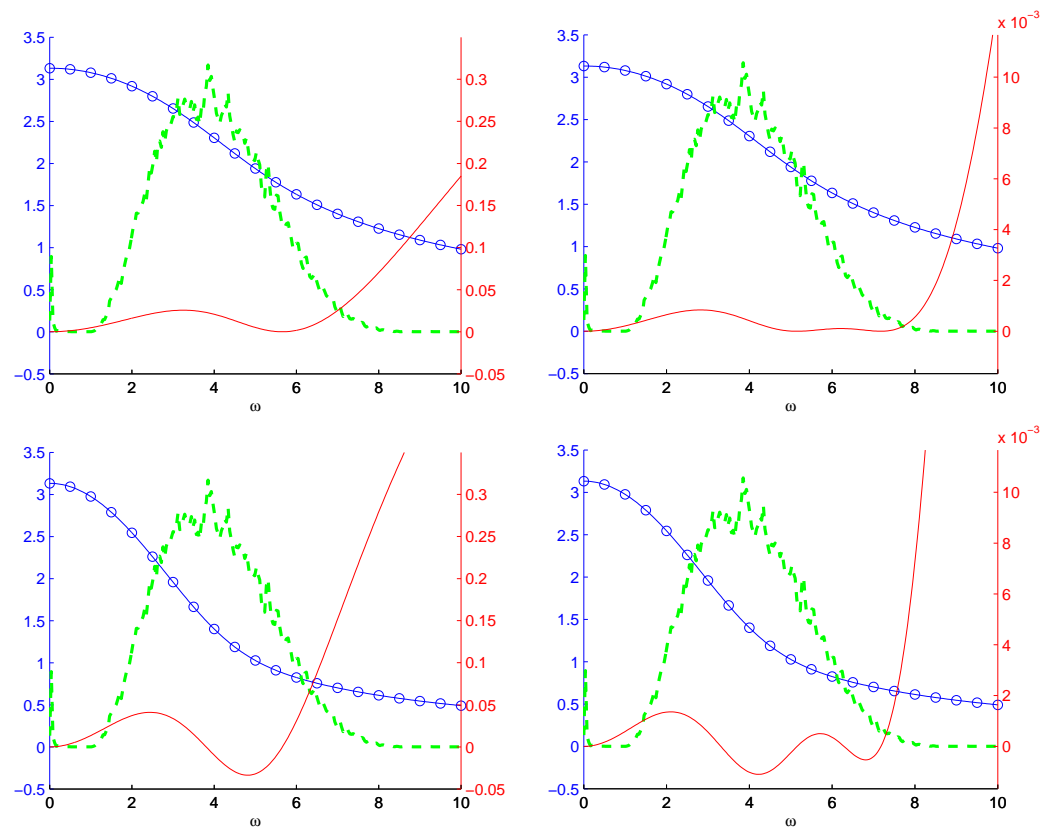

Figure 22: At the left and the right we show plots for the case of one and two vertical profiles respectively, with optimal choices of the parameters. The dashed curve is the power spectrum of the initial signal. In the upper plots is shown the exact phase velocity (solid with circles; left-hand axis) and the difference of the approximate phase velocity with the exact one (solid); in the plots below the same for the group velocity. Notice, that the scale for solid curves (right-hand axis) is 30 times finer for the 2-profiles approximation at the right.

$$
C_{t, a p p}(\omega)=\frac{\omega}{K_{a p p}(\omega)} .
$$

The difference between the two phase speeds is denoted by $\Delta C_{t}$

$$
\Delta C_{t}(\omega)=C_{t, a p p}(\omega)-C_{t, e x}(\omega) .
$$

Since the group speeds of the exact dispersion relation and the hyperbolic approximation coincide at the points $\kappa_{j}$, the group speed error

$$
\Delta V_{t}(\omega)=V_{t, a p p}(\omega)-V_{t, e x}(\omega)
$$

vanishes for $\omega=v_{j}$. Actually, in between such successive exact values, there is another frequency for which the error vanishes; as seen in Fig. 22 , the (first) additional exact frequency for which the group speeds are the same is quite close to the peak frequency of the shown spectra.

Fig. 22 illustrates the peculiarities in the choice of the optimal values $v_{j}$. Intuitively, we would like the approximate velocities to be accurate, 
i.e. small $\Delta C$ and $\Delta V$ for frequencies for which the spectrum is large. But for applications with a broad spectrum as in this example, the best value is not obvious. Besides that, we have only very limited intuition what the effect of changes in phase or group speed is on the actual evolution of the waves. The optimal values as illustrated here show that the speed errors are spread over the relevant frequency interval, with small errors near the peak frequency.

Note that for the 1-profile approximation the error $\Delta V$ is much larger than $\Delta C$ in the tail of the spectrum close to $\omega=10$ : the error $\Delta V$ is comparable to the actual value, while the error $\Delta C$ is approximately 20 $\%$ near $\omega=10$. Since $\Delta V$ vanishes also in a point close to the peak frequency, we observe that an optimal $v$-value gives a group speed curve that has minimal error over a rather large frequency range, but the error increases much faster than the error in phase speed for higher frequencies. The 2-profile approximation behaves similarly, but the errors are a factor of 20 or more smaller than for the 1-profile approximation in the interval of interest.

We have investigated other reasonable 'ad-hoc' optimization criteria, each of which is aimed to reduce the velocity error (either phase or group speed) over the whole relevant frequency interval. We found that the proposed kinetic optimization criterion performs better than these criteria, in addition to the fact that it is a direct consequence of the minimization property of the kinetic energy. For details see [23].

\subsection{TEST CASE}

In this section we consider the case of a focussing wave group (FWG). We describe in this section the main characteristics of the initial signal and spectrum, consider the evolution with the accurate AB-equation for linear and non-linear evolutions; in Section 4.5 we will use these together with the measurement at the focussing point to see the result of the simulation with the optimized VBM. For another realistic test case of a focussing wave group and performance of the VBM, see report [23].

\subsubsection{Focussing wave group (FWG)}

The test case is an example of a constructed wave field that was designed at MARIN, the Maritime Hydrodynamic Laboratory Netherlands, to generate high waves in a long wave tank. The design is to exploit dispersive focussing: short period, small amplitude waves are generated at a waveflap, followed by successively higher and longer period waves that catch up with the slower shorter waves at a predetermined position in the tank. This dispersive focussing requires a broad spectrum, and different speeds of different frequency components de- 

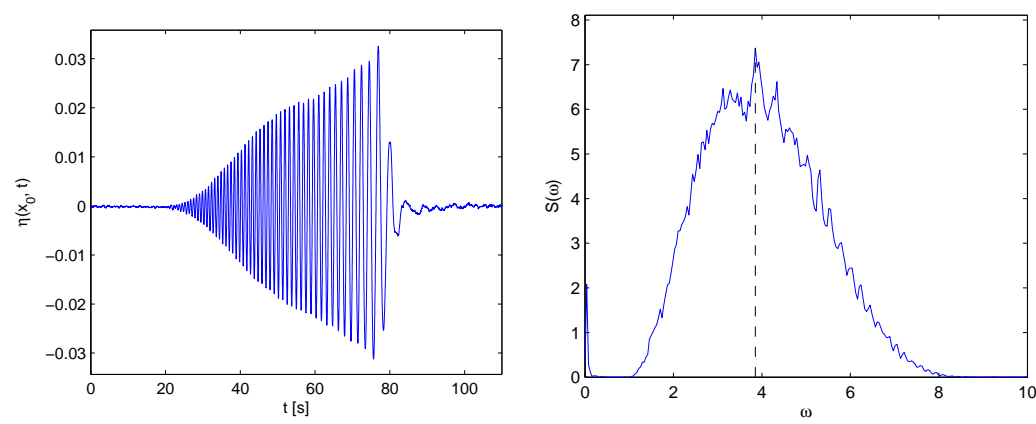

Figure 23: Signal (left) and its power spectrum (right) at $X_{0}=0$ for the Focussing Wave Group.

termine the focussing process in a critical way. Hence, the behaviour will be most sensitive to perturbations in the phase speed, which is why we choose this example.

For this case real laboratory measurements in a wavetank with depth of $1 \mathrm{~m}$ are available: the surface elevation at $x=X_{0}^{\prime}=10 \mathrm{~m}$ from the waveflap and at the (designed) focussing point $x=X_{1}^{\prime}=50 \mathrm{~m}$. We shift in our numerical model the starting position to the position $x=$ $X_{0}=0 \mathrm{~m}$ and accordingly the measurement position to $x=X_{1}=40 \mathrm{~m}$.

In Fig. 23, we present the time signal at $X_{0}$ together with the power spectrum. Observe that the spectrum is broad. The test case is rather extreme: just downstream of the focussing point some breaking (white capping) was observed.

\subsubsection{Accurate simulation of the focussing process}

The MARIN measurement of the elevation at $X_{1}$ is the only available information downstream of $X_{0}$. This gives only little information, which is why we performed additional numerical calculations with a very accurate and efficient model for uni-directional waves, i.e. the AB-equation derived by Van Groesen \& Andonowati [12] (see [13] for numerical results). The results with the non-linear version of this code - which uses the exact dispersion - will be used to compare with VBM calculations in the next section.

First, we show the result of a nonlinear evolution with a pseudospectral implementation of the AB-equation, downstream from the measured elevation signal at $X_{0}$.

In Fig. 24 at the left we show at $X_{1}$ the calculated signal and the measured signal. These results show that the simulations are remarkably accurate, even for such an extreme case as the FWG. In measurements and simulations the spectrum changes during the evolution due to nonlinear effects. In fact, detailed analysis of the simulations show 

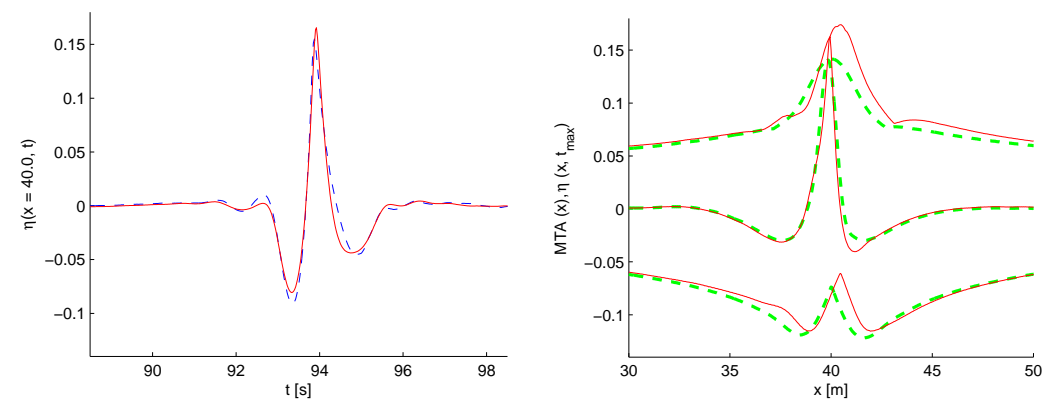

Figure 24: At the left the measured signal (dashed) at the focussing point $X_{1}=40 \mathrm{~m}$ is compared to the signal given by AB-nonlinear simulation (solid). At the right we compare MTA-s and wave elevation at the moment of the maximal wave amplitude at $X_{1}$ : by AB-linear (dashed) and by AB-nonlinear (solid). For this rather extreme case of the FWG we observe that the linear simulation gives a result that is quite close to the nonlinear simulation.

that long- and short-wave generation takes place very close to the focussing point. Since this thesis deals mostly with improvement of the linear dispersive effects of the VBM, we also consider the linear evolution of the initial signal which is the evolution according to the exact dispersion; this can be done with the linearized AB-equation. In Fig. 24 at the right we compare it to the non-linear simulation. Remarkably, also for this linear simulation the wave elevation at the time of focussing is quite similar to the non-linear one, albeit the amplitude is somewhat less, despite the fact that nonlinear effects do play a role in this test case.

The numerical simulations - different from the available measurements - also provide information at any point in between $X_{0}$ and $X_{1}$. To get condensed information of that evolution, we consider the maximal temporal amplitudes (MTA): the maximal crest height (positive curve) and the minimal trough depth (negative curve) at each position. The results are shown in Fig. 24, and we will use these to compare the MTA calculated with the VBM in the next section.

\subsection{OPTIMIZED VBM SIMULATIONS}

In this section we use the optimal parameter values found from the optimization method in Section 4.3 for FWG. We performed linear and nonlinear numerical simulations using these optimal values in the Finite Element implementation of VBM. In Subsection 4.5.1 we present the results of the VBM calculated time signals at the focussing points for the optimal values. In Subsection 4.5.2 we provide the results when looking at the downstream evolution as measured by the 

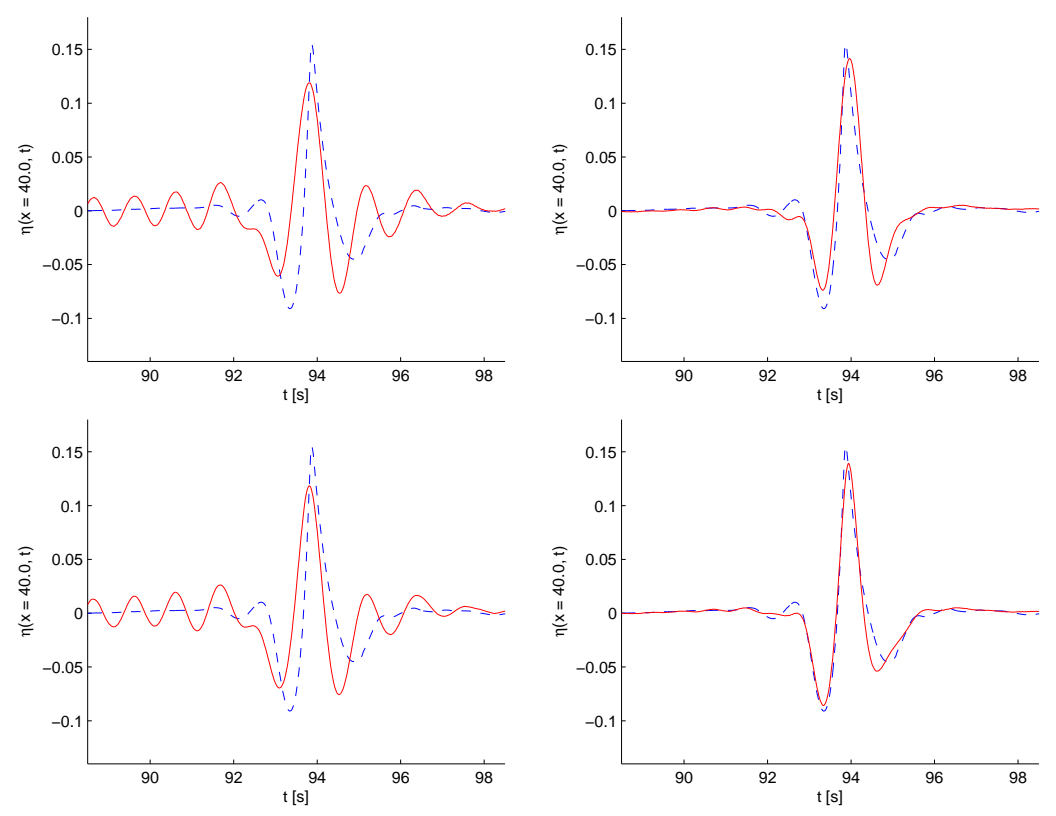

Figure 25: Comparison of temporal signals at $X_{1}$ for the FWG: at the top linear and at the bottom non-linear simulations. At the left OVBMsimulation with the 1-profile approximation (solid) compared to the measurement (dashed), and at the right for the optimized 2-profile approximation.

MTA-s. In Subsection 4.5.3 we provide results of simulations with two other codes, MIKE 21 BW [33] and SWASH [40].

\subsubsection{Signal at focussing point}

We used a Finite Element implementation of the optimized VBM to calculate the time signal at the focussing point, with the optimal values obtained from the kinetic energy optimization, as given in Table 3. The result is shown in Fig. 25. To compare the results, we also depicted in the same plot the signals of the measurement. It can be observed that in the case of the 1-profile approximation the main wave is relatively well represented, but that certain frequency components disturb the signal before and after the time of focussing. In the case of the 2-profile approximation the signal comes much closer to the experimental signal.

\subsubsection{Maximal temporal amplitudes}

Understandably, the additional oscillations in the VBM time signal with the 1-profile simulation, as seen in Fig. 25, also have effects on 

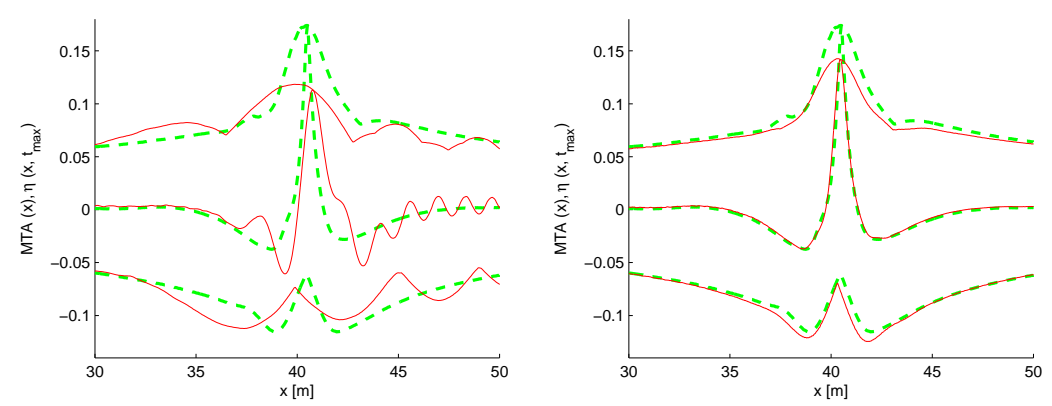

Figure 26: Nonlinear simulations to compare the MTA-s and a plot of the spatial wave profile at time $t=94.2 \mathrm{~s}$. At the left for OVBM with the 1-profile approximation (solid), at the right for OVBM with the 2-profile approximation (solid), compared to the AB-simulation (dashed).

the spatial profile. This is visible in Fig. 26 as well. In these spatial plots we compare the wave profile and the curves of Maximal Temporal Amplitudes for each simulation.

It is visible that the 2-profile VBM approximation gives good results, compared to the exact dispersion calculations. It is noticeable, that the position of simulated focussing, where the maximal amplitude wave appears, is happening slightly behind the measurement point $X_{1}=$ $40 \mathrm{~m}$.

Different additional measures, showing the optimality of the $v$-parameter choice in the 1-profile case, according to the kinetic energy minimization principle, are given in report [23].

\subsubsection{Simulation results with other codes}

The broad spectrum of the test case considered in this section is the main difficulty for accurate simulations: at the focussing position all waves should contribute maximally, i.e. with the same vanishing phase at the same time. This requires that the propagation speed is accurate enough for each of the waves to travel the distance from generation to focussing position, here $X_{1}=40 \mathrm{~m}$, in the correct time. To obtain the maximal wave height accurately, also the contribution of short waves is important. We present here result of simulations with two other codes. MIKE 21 BW [33] uses the 'enhanced' linear dispersion characteristics originally derived by Madsen et al. [28] and Madsen \& Sørensen [29]. The maximum depth to wavelength ratio is $\frac{1}{2}$, meaning that waves of wavelength smaller than two times the depth are not, or poorly, simulated. In Fig. 27, the MIKE result (solid line) shows that the largest wave occurs at the correct time, but the maximal height is poorly ap- 


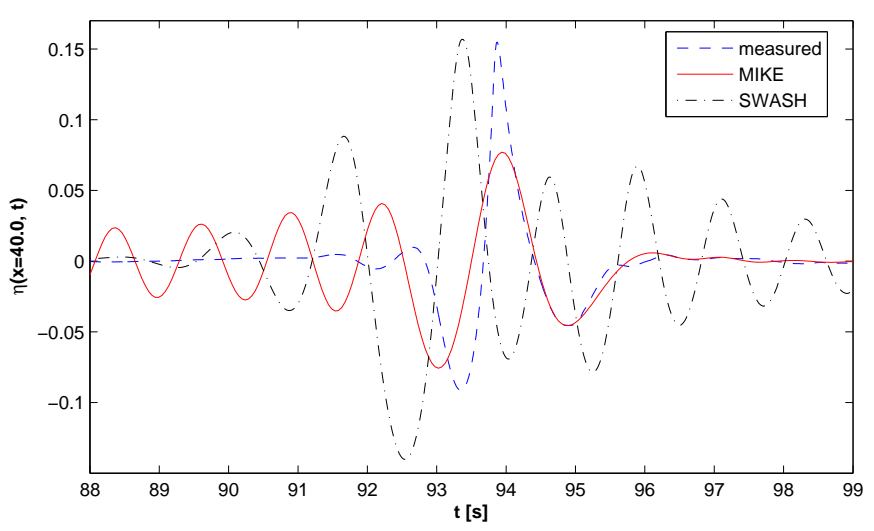

Figure 27: Shown are the time signals at $X=40 \mathrm{~m}$ of the measurement (dashed) and two simulations: MIKE21BW (solid) and SWASH (dash-dot).

proximated, seemingly because a substantial part of the waves arrive too early.

The dispersion in SWASH [40, 44] can be influenced by choosing a division in various layers. Because an optimal division is not obvious, we tried various choices. For a division of 3 layers given by 2.5, 5 and $92.5 \%$ (percentage of depth measured from the free surface), small variations around this division showed little difference in the results. The result of the simulation for the given division (dashed-dot) shows a large wave with much too low trough depth; this wave occurs close to the focussing time but is preceded and followed by substantial additional waves. It is clear that the energy of this simulated wave is much too high, but the reason for this is not clear. Possibly, the influxing of the time signal at the origin is not accurate enough.

\subsection{CONCLUSIONS AND REMARKS}

Although we provide some details about the numerical implementation in Subsection 4.2.3, the analysis in this thesis is concentrated on errors caused by the model equations, and not on numerical accuracy errors. The numerical simulations are performed for the exact dispersion with a spectral AB-code; for the VBM simulation we used the FEM-implementation with sufficiently fine grid.

The freedom in the Variational Boussinesq Model (VBM) to choose the vertical profiles of the fluid potential was exploited in this chapter. We discovered that taking progressively larger superpositions of Airy profiles that approximate the fluid potential, drastically improves the results. Already with a 2-profile approximation the dispersive charac- 
teristics of VBM are very close to the exact dispersion, as seen in Fig. 22.

For the effective wave numbers $\kappa_{j}$-s, used in the VBM, Airy theory provides the exact dispersion. Using the minimum kinetic energy principle it was shown that this criterium leads to wave numbers $\kappa_{j}$-s in an optimal way, minimizing the kinetic energy error, simultaneously minimizing the error in phase and group speed. It should be noted that to determine the optimal values we used properties of the influxed signal (or of an initial wave profile); this then determines the specific dispersive properties used in the simulation. This is different from most other wave models for which the simulation code does not depend on the specific wave to be simulated.

A good simulation of the focusing process requires all participating waves to evolve accurately. The result of the optimal performance is shown in Fig. 25 and Fig. 26. The spatial and temporal positioning of the maximal (focused) wave is rather accurate already for the 1-profile approximation in the OVBM. And further improvement by using the 2-profile approximation allows to fix the remaining focussing errors in the higher frequencies and capture the shape and amplitude of the maximal wave in a good way.

At this moment the optimization method has already been extended to be applicable to waves above bathymetry. Results of irregular waves evolving over a slope [2] are published already, and of harmonics to calculate reflection from slopes [25] will be published shortly. This will be also the topic of the next chapter.

\section{Addendum}

Lately M. Zijlema provided us with a new SWASH simulation of the focussing wave group. Fig. 28 shows his result obtained with different settings of the software, demonstrating that the SWASH model can be fine-tuned to obtain results better than those shown in Figure 27. We are very grateful for his reaction to our request to investigate the poor behaviour that we obtained. 


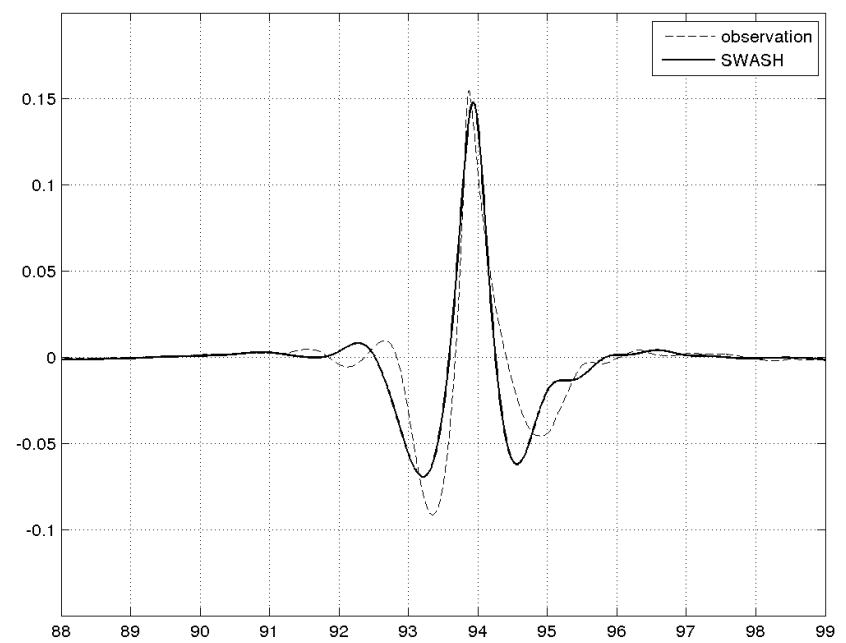

Figure 28: Shown are the time signals at $X=40 \mathrm{~m}$ of the measurement (dashed) and the later simulation by SWASH (solid). 

[1] D. Adytia, E. van Groesen, The Variational 2D Boussinesq model for wave propagation over a shoal, in: International Conference on Developments in Marine CFD, 18 - 19 November 2011, Chennai, India.

[2] D. Adytia, E. van Groesen, Optimized Variational 1D Boussinesq modelling of coastal waves propagating over a slope, Submitted.

[3] W.J.D. Bateman, C. Swan, P.H. Taylor, On the efficient numerical simulation of directionally spread surface water waves, J. Comput. Phys. 174 (2001) 277-305.

[4] A.M. Balk, A Lagragian for water waves, Phys. Fluids 8 (1996) 416-419.

[5] J. Boussinesq, Théorie des ondes et des remous qui se propagent le long d'un canal rectangulaire horizontal, en communiquant au liquide contenu dans ce canal des vitesses sensiblement pareilles de la surface au fond, J. Math. Pures Appl. 17 (1872) 55-108.

[6] L.J.F. Broer, On the Hamiltonian theory of surface waves, Appl. Sci. Res. 30 (1974) 340.

[7] D. Clamond, D. Dutykh, Practical use of variational principles for modeling water waves, Physica D (2011), In press.

[8] W. Craig, C. Sulem, Numerical simulation of gravity waves, J. Comput. Phys. 108 (1993) 73-83.

[9] M. Dingemans, Water wave propagation over uneven bottoms (World Scientic) 1 \& 2 (1997).

[10] A.E. Green, P.M. Naghdi, A derivation of equations for wave propagation in water of variable depth, J. Fluid Mech. 78 (1976) 237-246.

[11] E. van Groesen, Jaap Molenaar, Continuum Modeling in the Physical Sciences, in: Mathematical Modeling and Computation (SIAM) 13 (2007), 236 pp.; ISBN-13: 978-0-898716-25-2.

[12] E.van Groesen, Andonowati, Variational derivation of KdV-type of models for surface water waves, Phys. Lett. A 366 (2007) 195201. 
[13] E. van Groesen, Andonowati, L. She Liam, I. Lakhturov, Accurate modelling of uni-directional surface waves, J. Comput. Appl. Math. 234 (2010) 1747-1756.

[14] E. van Groesen, Andonowati, Fully dispersive dynamic models for surface water waves above varying bottom, Part 1: Model equations, Wave Motion 48 (2011) 658-667.

[15] E. van Groesen, I. van der Kroon, Fully dispersive dynamic models for surface water waves above varying bottom, Part 2: Hybrid spatial-spectral implementations, Wave Motion 49 (2012) 198-211.

[16] J.W. Kim, K.J. Bai, R.C. Ertekin, W.C. Webster, A derivation of the Green-Naghdi equations for irrotational flows, J. Eng. Math. 40 (2001) 17-42.

[17] J. T. Kirby, Nonlinear, dispersive long waves in water of variable depth, in: Gravity Waves in Water of Finite Depth (Computational Mechanics Publications) 10 (1997) 55-125.

[18] J. T. Kirby, Boussinesq models and applications to nearshore wave propagation, surfzone processes and wave-induced currents, in Advances in Coastal Modelling (Elsevier) 67 (2003) 1-41.

[19] G. Klopman, M. W. Dingemans, E. van Groesen, A variational model for fully non-linear water waves of Boussinesq type, in: Proceedings of the International Workshop on Water Waves and Floating Bodies, 2005.

[20] G. Klopman, M. W. Dingemans, E. van Groesen, Propagation of wave groups over bathymetry using a variational Boussinesq model, in: Proceedings of the International Workshop on Water Waves and Floating Bodies, 2007.

[21] G. Klopman, M. W. Dingemans, Reflection in variational models for linear waves, Wave Motion 47 (2010) 469-489.

[22] G. Klopman, E. van Groesen, M. W. Dingemans, A variational approach to Boussinesq modelling of fully nonlinear water waves, J. Fluid Mech. 657 (2010) 36-63.

[23] I. Lakhturov, E. van Groesen, Optimized Variational Boussinesq Modelling; part 1: Broad-band waves over flat bottom, Internal report, University of Twente, The Netherlands (2010). See http:// eprints.eemcs. utwente.nl/.

[24] I. Lakhturov, D. Adytia, E. van Groesen, Optimized Variational ID Boussinesq Modelling for broad-band waves over flat bottom, Wave Motion 49 (2012) 309-322 
[25] I. Lakhturov, E. van Groesen, Reflection calculations for waves above a slope using Optimized Variational 1D Boussinesq simulations, In preparation.

[26] J.C. Luke, A variational principle for a fluid with a free surface, J. Fluid Mech. 27 (1967) 395-397.

[27] P. Lynett, P. L.-F. Liu, Linear analysis of the multi-layer model, Coast. Eng. 51 (2004) 439-454.

[28] P.A. Madsen, R. Murray, O. R. Sørensen, A new form of the Boussinesq equations with improved linear dispersion characteristics, Coast. Eng. 15 (1991), 371-388.

[29] P.A. Madsen, O. R. Sørensen, A new form of the Boussinesq equations with improved linear dispersion characteristics. Part 2. A slowly-varying bathymetry, Coast. Eng. 18 (1992), 371-388.

[30] P. A. Madsen, H. A. Schäffer, Higher-order Boussinesq-type equations for surface gravity waves: derivation and analysis, Phil. Trans. R. Soc. Lond. A 356 (1998), 3123-3181.

[31] P. A. Madsen, H. A. Schäffer, A review of Boussinesq-type equations for gravity waves, in: World Scientic, Advances in Coastal and Ocean Engineering (World Scientic) 5 (1999) 1-95.

[32] P. A. Madsen, D. R. Fuhrman, High-order Boussinesq-type modelling of nonlinear wave phenomena in deep and shallow water, in: Advances in numerical simulation of nonlinear water waves (World Scientic) 5 (2010) 245-285.

[33] MIKE by DHI software, http://mikebydhi.com/

[34] J.W. Miles, On Hamilton's principle for surface waves, J. Fluid Mech. 83 (1977) 153-158.

[35] O. Nwogu, Alternative form of Boussinesq equations for nearshore wave propagation, J. Waterw. Port C-ASCE 119 (1993) 618-638.

[36] E. Pelinovsky, Hydrodynamics of tsunami waves, Waves in Geophysical Fluids, in: CISM Courses and Lectures (Springer) 489 (2006) $1-48$.

[37] A.C. Radder, Hamiltonian dynamics of water waves, Adv. Coast. Ocean Eng. 4 (1999) 21-59.

[38] R. Salmon, Hamiltonian fluid mechanics, Ann. Rev. Fluid Mech. 20 (1988) 225-256. 
[39] H. A. Schäffer, P. A. Madsen, Further enhancements of Boussinesq-type equations, Coast. Eng. 26 (1995) 1-15.

[40] SWASH, http: //swash. sourceforge.net/

[41] Liwei Xu, P. Guyenne, Numerical simulation of threedimensional nonlinear water waves, J. Comput. Phys. 228 (2009) 8446-8466.

[42] V.E. Zakharov, Stability of periodic waves of finite amplitude on the surface of a deep fluid, J. Appl. Mech. Tech. Phys. 2 (1968) 190194.

[43] V.E. Zakharov, E.A. Kuznetsov, Hamiltonian formalism for nonlinear waves, Usp. Fiz. Nauk 167 (1997) 1137-1168.

[44] M. Zijlema, G.Stelling, P. Smit, SWASH: an operational public domain code for simulating wave fields and rapidly varied flows in coastal waters, Coastal Eng. 58 (2011) 992-1012.

[45] Z. L. Zou, Higher order Boussinesq equations, Ocean Eng. 26 (1999) 767-792. 
In the previous chapters we searched for optimal dispersive properties of the VBM over flat bottom and showed results of comparing the experimental data with VBM calculations. In this chapter we calculate reflection coefficients of the model for the Booij's test case [1] and compare the obtained results with results of Porter \& Porter [17] and Klopman \& Dingemans [11]. Following [13], we also derive the analytical reflection-capable model based on WKB approximation and use it to obtain a reflection curve. VBM- and WKB-reflection results, being obtained with a simpler approach, are close to ones obtained by Porter \& Porter and Klopman \& Dingemans. We also do additional simulations, varying a different parameter than in the previous works.

\subsection{INTRODUCTION}

In the previous Chapters 3 and 4 we searched for optimal dispersive properties of the 1-D Variational Boussinesq model (VBM) over flat bottom and, using a finite element numerical code, investigated its quality, and showed results of comparing the VBM calculations to the experimental data. It also contained a comparison to other two other well-known models, MIKE and SWASH, as well as a comparison to the $\mathrm{AB}$-equation, recently derived and investigated by van Groesen et al. $[6,7]$. The latter model has already acquired a generalization for the varying bottom $[8,9]$.

In this chapter we use the VBM over varying bottom in order to obtain coefficients of the reflection from a plane slope bathymetry. In comparison with the previous chapter, we limit the model to the VBM with one profile (16) here. See Subsection 2.1.2 for the PDE system and Subsection 2.1.4 for the numerical implementation details. This is possible because of the numerical experiment setup, where only one time period for the influxed harmonic signal is used. Nevertheless, the model is suitable for wide range of bathymetries and wave types. The experimental layout, in turn, follows Booij's layout [1], later used by Porter \& Porter [17] and Klopman \& Dingemans [10, 11]. As an extension, we also use the model in a slightly different setting, in order to obtain the dependence of the reflection coefficient from the different parameter.

In addition, we derive a WKB-type of approximation and use it to obtain the reflection coefficients. This is an analytic approach, where the reflected wave is expressed by an integral formula via the influxed 
signal with a bathymetry-derived function as density kernel. As it is shown further, both approaches give remarkably good results.

The outline of the chapter is as follows. Further in the introduction we recall the derivation of the reflection coefficient from a vertical step in the bottom profile, as well as the derivation of the classical WKB approximation. Both are based on the well-known linear shallow water equation. In Section 5.2 we start with the same equation to derive the reflection-capable WKB-approximation. The next Section 5.3 gives details of the experiment setup, of the reflection coefficient calculations according to the both employed models, WKB and VBM, and compares them to the results obtained by Klopman \& Dingemans [11]. In Section 5.4 we provide some remarks and conclusions.

\subsubsection{Reflection from a step in the bathymetry}

We start out with the well-known one-dimensional bidirectional linear shallow water equation:

$$
\partial_{t}^{2} \eta=\partial_{x}\left(c^{2} \partial_{x} \eta\right)
$$

where $\eta(x, t)$ is the wave elevation depending on the spatial variable $x$ and the temporal variable $t$, and $c(x)=\sqrt{g h(x)}$ is the phase speed, which depends on the depth function $h(x) ; g$ is the gravitational acceleration.

Consider a jump in a bottom profile. How much reflection for a sinusoidal wave will provide such discontinuity? We recall how to find the reflection and transmission coefficients, making use of the linear shallow water equation (76).

The bottom geometry and incident waves are schematically shown in Fig. 29. The depth profile is given by $h(x)=h_{0}$ for $x<0$ and $h(x)=h_{1}$ for $x>0$, so the depth function jumps discontinuously with the relative change $h_{1} / h_{0}$. The incident wave is a sinusoid with amplitude $A$, propagating from the left. It is partially reflected from the discontinuity, making up a sinusoid with amplitude $B$, propagating to the left, and partially transmitted with amplitude $C$ to the right.

We write solutions as $\eta_{0}$ and $\eta_{1}$ - to the left and to the right of the bathymetry jump, correspondingly:

$$
\begin{aligned}
& \eta_{0}=\left(A e^{i k_{0} x}+B e^{-i k_{0} x}\right) \cdot e^{-i \omega_{0} t} \\
& \eta_{1}=C e^{i k_{1} x} \cdot e^{-i \omega_{0} t}
\end{aligned}
$$

where $k_{0}$ and $k_{1}$ are wave numbers to the left and to the right.

The reflection and transmission coefficients are expressed as

$$
R=\frac{B}{A}, \quad T=\frac{C}{A} .
$$




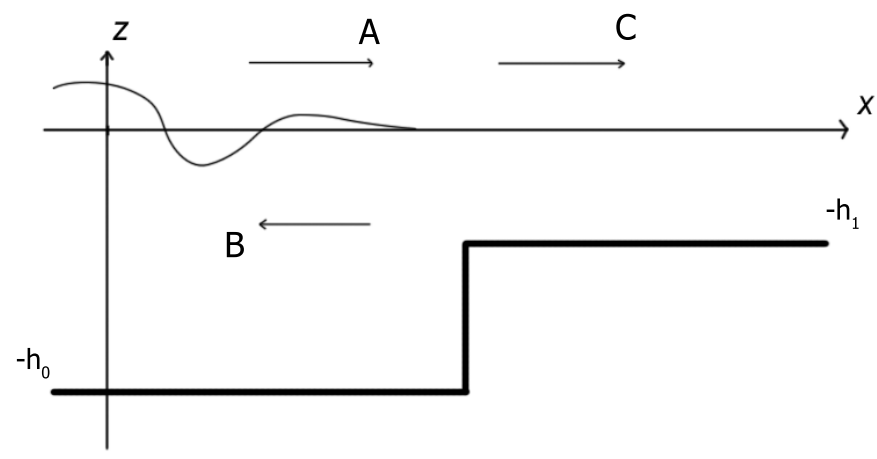

Figure 29: Reflection induced by a discontinuity in bathymetry. Bottom profile rises suddenly from $-h_{0}$ to $-h_{1}$. A sinusoidal wave with the amplitude $A$ coming from the left is partially reflected with the amplitude $B$ to the left and partially transmitted with the amplitude $C$ to the right.

It is necessary to require that there is no disconiuity in the wave profile:

$$
\eta_{0}=\left.\eta_{1}\right|_{x=0}
$$

which is immediately rewritten as $A+B=C$ or

$$
1+R=T .
$$

The second matching condition follows from the requirement that the flux is continuous at $x=0$ :

$$
c_{0}^{2} \partial_{x} \eta_{0}=\left.c_{1}^{2} \partial_{x} \eta_{1}\right|_{x=0} .
$$

Indeed, considering equation (76), if this expression has a jump in the zero point, there will be an infinite acceleration $\partial_{t}^{2} \eta$ of a wave surface. Rewriting this condition in the form $h_{0} \partial_{x} \eta_{0}=\left.h_{1} \partial_{x} \eta_{1}\right|_{x=0}$, we see that $A k_{0} h_{0}-B k_{0} h_{0}=C k_{1} h_{1}$, or

$$
k_{0} h_{0}-R k_{0} h_{0}=T k_{1} h_{1} .
$$

In the long-wave limit wave number $k$ is expressed through frequency $\omega$ (which is constant in our case) and depth $h(x)$ as $k=\omega / \sqrt{g h}$, so the ratio of wave numbers to the right and to the left is $k_{1} / k_{0}=$ $\sqrt{h_{0} / h_{1}}$. After simple algebraic transformations we obtain from (80) and (82):

$$
R=\frac{1-\sqrt{h_{1} / h_{0}}}{1+\sqrt{h_{1} / h_{0}}}, \quad T=\frac{2}{1+\sqrt{h_{1} / h_{0}}}
$$


These expressions conform to the reflection and transmission coefficients given in [4] (see expressions (2.296) at p. 138). As $\sqrt{h_{1} / h_{0}}=c_{1} / c_{0}$, we can also rewrite them through the phase speed:

$$
R=\frac{1-c_{1} / c_{0}}{1+c_{1} / c_{0}}, \quad T=\frac{2}{1+c_{1} / c_{0}},
$$

or, equivalently,

$$
R=\frac{c_{0}-c_{1}}{c_{0}+c_{1}}, \quad T=\frac{2 c_{0}}{c_{0}+c_{1}},
$$

which are the same expressions as in [16].

\subsubsection{Classical WKB-approximation}

Let us recall the derivation of the WKB-approximation. We start from the linear bidirectional shallow water equation (76). For constant depth $c$ this equation becomes $\partial_{t}^{2} \eta=c^{2} \partial_{x}^{2} \eta$ and the solution is the superposition of a right-propagating profile $F$ and a left-propagating profile G:

$$
\eta(x, t)=F\left(t-\frac{x}{c}\right)+G\left(t+\frac{x}{c}\right) .
$$

Let us derive the WKB-approximation for the equation (76), but only for the right-propagating wave. Assume $c(x)$ is a slowly varying function. Take the following Ansatz for the solution:

$$
\eta(x, t)=\rho(x) F(\theta(x, t)),
$$

ht5rrtfere $\rho$ is a slowly varying 'amplitude' and $\theta$ is a phase function to be found. Substitute this Ansatz into (76) and obtain the equation

$$
r_{0} F+r_{1} F^{\prime}+r_{2} F^{\prime \prime}=0,
$$

where the coefficients are

$$
\begin{aligned}
& r_{0}=-2 c c^{\prime} \rho^{\prime}-c^{2} \rho^{\prime \prime}=-\left(c^{2} \rho^{\prime}\right)^{\prime} \\
& r_{1}=\rho \theta_{t t}-2 c^{2} \rho^{\prime} \theta_{x}-2 c c^{\prime} \rho \theta_{x}-c^{2} \rho \theta_{x x} \\
& r_{2}=\rho \theta_{t}^{2}-c^{2} \rho \theta_{x}^{2}=\rho\left(\theta_{t}-c \theta_{x}\right) \underbrace{\left(\theta_{t}+c \theta_{x}\right)} .
\end{aligned}
$$

Taking into account only a right-propagating wave, i.e. neglecting the part with the underbrace, obtain the solution for the equation $r_{2}=$ 0: $\theta_{t}-c \theta_{x}=0$, i.e.

$$
\theta(x, t)=t-\tau(x)=t-\int_{0}^{x} \frac{d \zeta}{c(\zeta)} .
$$

Here $\tau(x)$ is a 'delay' or 'travel-time' function, which depends only on bathymetry and shows how long it takes for a wave to shift to the 
point $x$. Now that $\theta_{x}=-1 / c$ and $r_{1}=2 c \rho^{\prime}+c^{\prime} \rho$, obtain the solution of the equation $r_{1}=0$ :

$$
\rho(x)=\frac{A}{\sqrt{c(x)}},
$$

with a certain constant $A$, which we incorporate into the profile $F$ in (87).

Functions $c$ and $\rho$ are slowly varying with respect to $x$. As $r_{0}$ contains only second-order components, we neglect it. Then the WKBapproximation of the right-propagating solution for equation $(76)$ is

$$
\eta(x, t)=\frac{F(t-\tau)}{\sqrt{c}}=\frac{F\left(t-\int_{0}^{x} \frac{d \zeta}{c(\zeta)}\right)}{\sqrt{c(x)}} .
$$

Notice, this is also the solution of the unidirectional equation

$$
\partial_{t} \eta=\sqrt{c} \partial_{x}(\sqrt{c} \eta)
$$

which we will call further the WKB-equation, as in [5].

We have derived the classical WKB-approximation. In the next section we use it as the starting point for the derivation of the reflective WKB-model.

\subsection{REFLECTION-CAPABLE WKB-APPROXIMATION}

Following [13], we derive here a WKB-approximation capable of generating reflection from bathymetry features. In Section 5.3 we will compare these analytical results to the reflection results of the VBM.

We start from the linear shallow water equation (76), rewriting it in the form:

$$
\left(\partial_{t}+D\right)\left(\partial_{t}-D\right)=\left(\partial_{t}^{2}-D^{2}\right) \eta=b \eta
$$

with operator $D$ the WKB-operator from the equation (95):

$$
D=\sqrt{c} \partial_{x} \sqrt{c}
$$

and $b$ the operator

$$
b=\partial_{x} c^{2} \partial_{x}-\sqrt{c} \partial_{x} c \partial_{x} \sqrt{c} .
$$

It is notable that action of this operator is nothing else, but multiplication:

$$
b \eta=b \cdot \eta=-\frac{1}{4}\left(c^{\prime 2}+2 c c^{\prime \prime}\right) \cdot \eta=\frac{1}{2} c^{3 / 2} \frac{d}{d x}\left[\frac{c^{\prime}}{\sqrt{c}}\right] \cdot \eta .
$$

Let us search for a solution $\eta$ of (96) in the form

$$
\eta=\eta_{0}+\eta_{1}+\eta_{2}+\ldots
$$


where $\eta_{0}$ is the WKB-solution (94), propagating to the right, so the left part of equation (96) vanishes for it. Taking the first two $\eta$-components, we get from (96):

$$
\left(\partial_{t}+D\right)\left(\partial_{t}-D\right) \eta_{1}=b \eta_{0}+b \eta_{1}
$$

As this equation, generally speaking, cannot be solved analytically, we neglect the higher-order term $b \eta_{1}$, rewriting the equation as

$$
\left(\partial_{t}+D\right)\left(\partial_{t}-D\right) \eta_{1}=b \cdot \eta_{0}
$$

This one already has a solution, expressed with an integral formula. If we continue the process, we will end up with the similar expression

$$
\left(\partial_{t}+D\right)\left(\partial_{t}-D\right) \eta_{i+1}=b \cdot \eta_{i}
$$

To solve (102), we rewrite it as the equivalent system with $\xi$ the wave that satisfies a forced left-travelling wave equation:

$$
\left\{\begin{array}{l}
\left(\partial_{t}+D\right) \xi=b \eta_{0} \\
\left(\partial_{t}-D\right) \eta_{1}=\xi
\end{array}\right.
$$

and solve equations as initial value problems. The profile of the classical WKB-solution $\eta_{0}$ (94) is given for the starting time $t=0$, but other functions $\eta_{1}$ and $\xi$, forming the reflected wave, are zero initially.

We multiply these equations by $\sqrt{c(x)}$ and change variables from $x$ to the travel-time function $\tau(x)$ according to the expression (92) in order to obtain simple transport equations. Denoting $\overline{\eta_{i}}=\sqrt{c} \eta_{i}$ and $\bar{\xi}=\sqrt{c} \xi$, the system can be rewritten as

$$
\left\{\begin{array}{l}
\left(\partial_{t}+\partial_{\tau}\right) \bar{\xi}(\tau, t)=\bar{b}(\tau) \overline{\eta_{0}}(\tau, t) \\
\left(\partial_{t}-\partial_{\tau}\right) \overline{\eta_{1}}(\tau, t)=\bar{\xi}(\tau, t) .
\end{array}\right.
$$

Here $\tau(x)$ is the 'travel-time function', it gives the time needed for a signal to come from the zero point to the point $x$. Let us solve the first equation:

$$
\left(\partial_{t}+\partial_{\tau}\right) \bar{\xi}(\tau, t)=F(t-\tau) \bar{b}(\tau) .
$$

The function $F$ comes from expression (94) of the WKB-solution; notice that for $\tau(0)=0$ this function is a signal $s(t)$ at the origin (up to the factor $\sqrt{\mathcal{C}_{0}}$ ), and for different locations $x$ it is the same signal delayed with the period $\tau(x)$. The homogeneous solution of this non-homogeneous transport equation is zero due to initial condition, thus,

$$
\begin{aligned}
\bar{\xi}(\tau, t) & =\int_{0}^{t} F(t-\tau) \bar{b}(\tau+\sigma-t) d \sigma \\
& =F(t-\tau) \int_{\tau-t}^{\tau} \bar{b}(\sigma) d \sigma=F(t-\tau)[\bar{B}(\tau)-\bar{B}(\tau-t)] d \sigma
\end{aligned}
$$


where by $\bar{B}$ we denote the integral

$$
\bar{B}(\tau)=\int_{0}^{\tau} \bar{b}(\sigma) d \sigma
$$

For the same function, transferred to $x$-space, we give the name $B(x)$. After a few manipulations, we can obtain the following neat expression:

$$
\begin{aligned}
B(x) & =\int_{0}^{\tau(x)} \bar{b}(\zeta) d \zeta=\int_{0}^{x} \bar{b}(\zeta(\xi)) \frac{d \zeta}{d \xi} d \xi \\
& =\int_{0}^{x} b(\xi) \frac{1}{c(\xi)} d \xi=\frac{1}{2}\left[c^{\prime}(x)-c^{\prime}(0)\right]-\frac{1}{4} \int_{0}^{x} \frac{c^{\prime}(\xi)^{2}}{c(\xi)} d \xi .(108)
\end{aligned}
$$

Applying the similar procedure to the second equation of (105),

$$
\left(\partial_{t}-\partial_{\tau}\right) \overline{\eta_{1}}(\tau, t)=F(t-\tau)[\bar{B}(\tau)-\bar{B}(\tau-t)],
$$

we write down the solution

$$
\left.\overline{\eta_{1}}(\tau, t)=\int_{0}^{t} F(2 \sigma-t-\tau)\right)[\bar{B}(t+\tau-\sigma)-\bar{B}(t+\tau-2 \sigma)] d \sigma .
$$

The function $\eta_{1}$ can be first determined by formula with $\tau$-dependence:

$$
\left.\eta_{1}(\tau, t)=\frac{1}{\sqrt{\bar{c}(\tau)}} \int_{0}^{t} F(2 \sigma-t-\tau)\right)[\bar{B}(t+\tau-\sigma)-\bar{B}(t+\tau-2 \sigma)] d \sigma,
$$

and then transferred to the $x$-space. Another interesting form for this expression is

$$
\eta_{1}(\tau, t)=\frac{1}{\sqrt{\bar{c}(\tau)}}\left[\int_{\tau}^{\tau+t} F(t+\tau-2 \sigma) \bar{B}(\sigma) d \sigma-\frac{1}{2} \int_{\tau-t}^{\tau+t} F(-\sigma) \bar{B}(\sigma) d \sigma\right]
$$

As we are going to solve the signalling problem, the next step is to rewrite formula (III) via an influxed signal $s(t)$. Since $s(t)=\eta_{0}(0, t)=$ $\frac{F(t-\tau(0))}{\sqrt{c_{0}}}$ and $\tau(0)=0$ in the origin,

$$
F(t)=\sqrt{c_{0}} s(t),
$$

with $c_{0}=c(0)$ the phase speed in $x=0$. Now the integral formula can be immediately expressed in the form

$$
\eta_{1}(\tau, t)=\sqrt{\frac{\mathcal{c}_{0}}{\bar{c}(\tau)}} \int_{0}^{t} s(2 \sigma-t-\tau)[\bar{B}(\tau+t-\sigma)-\bar{B}(t+\tau-2 \sigma)] d \sigma . \text { (114) }
$$

In particular, in the origin the reflected wave is given by 
$\eta_{1}(0, t)=\int_{0}^{t} s(2 \sigma-t)[\bar{B}(t-\sigma)-\bar{B}(t-2 \sigma)] d \sigma=\int_{t / 2}^{t} s(2 \sigma-t) \bar{B}(t-\sigma) d \sigma$.

The last equality follows because $s(t)=0$ for $t<0$ and $\bar{B}(t-\sigma)=0$ in the chosen interval, as we regard the bottom function constant to the left of the origin. Rewritten as

$$
\eta_{1}(0, t)=\int_{0}^{t / 2} s(t-2 \sigma) \bar{B}(\sigma) d \sigma
$$

this expression can be interpreted geometrically. For instance, if we take the influx as a delta Dirac function $s(t)=\delta\left(t-t_{0}\right)$, delayed with some $t_{0}>0$, then the reflected signal will be

$$
\eta_{1}(0, t)=\int_{0}^{t / 2} \delta\left(t-t_{0}-2 \sigma\right) \bar{B}(\sigma) d \sigma=\bar{B}\left(\frac{t-t_{0}}{2}\right)
$$

for $t>t_{0}$, and zero otherwise. Now let $\xi_{0}$ be such point $x$, for which twice the travel time is $t-t_{0}$, i.e. $\tau\left(\xi_{0}\right)=\left(t-t_{0}\right) / 2$. Thus, $\bar{B}\left(\xi_{0}\right)$ is precisely the reflection at $x=0$ of the delta-function influx.

\subsection{NUMERICAL EXPERIMENT AND REFLECTION CURVE}

In our investigation of reflection in the VBM and WKB we follow the particular layout of the bathymetry profile, which stems from the note of Booij [1], and was used later by Porter \& Porter [17] and Klopman \& Dingemans [11]. Porter \& Porter give accurate reflection results for this bathymetry layout based particularly on the simulations with the full nonlinear equations. Klopman \& Dingemans use the time-harmonic linearized VBM and compare their results to the results of Porter \& Porter. In this chapter we compare our results against the results by Klopman \& Dingemans for being the most recent and using a related model.

It should be noted that our approach is more straightforward and simpler. They derive time-harmonic equations and use a heuristic procedure of choosing the normalization for the vertical cosh-profile in the VBM. In contrast, we use VBM as is - and obtain comparable results. The greatest advantage then is the capability of handling not only sinusoidal but arbitrary wave profiles.

As an addition, we also use the model in a slightly different setting, in order to obtain the dependence of the reflection coefficient on a different parameter. In contrast to Booij and Klopman \& Dingemans, who computed reflection curves dependent only on the bottom inclination, we also provide the reflection curves depending on the wave frequency. 


\subsubsection{Setup of the experiment}

Following experiments of the predecessors, we influx sinusoidal waves at the zero point $x=0$ :

$$
\eta_{0}=s(t)=A \sin \omega_{0} t
$$

where the amplitude $A=0.5 \mathrm{~m}$, so the wave height will be equal 1 $\mathrm{m}$, the angular frequency $\omega_{0}=\sqrt{k_{\infty} g}$, where $k_{\infty}$ is the infinite-depth wave number, equal to 1 , so the frequency $\omega_{0}=\sqrt{g} \approx \pi \approx 3.13 \mathrm{rad} / \mathrm{s}$. We ought to obtain the reflection coefficient $R=B / A$, which is the ratio of the reflected wave amplitude $B$ to the influxed wave amplitude $A$.

The layout of the experiment is schematically shown in Fig. 30; such bathymetry proflile is usually called a 'plane slope'. The one-dimensional domain spans the region from the leftmost (numerical) boundary $x=0$ to $x=x_{\text {right }}$, which is far enough not to generate reflections from the rightmost (numerical) boundary within the time span of our numerical experiment. The bottom rises from the depth $h_{0}$ at the left to $h_{1}$ at the right, passing through the plane slope between $x_{1}$ and $x_{2}$. Following the particular Booij's setup, we take these depth values equal to $0.6 \mathrm{~m}$ and $0.2 \mathrm{~m}$ respectively. The bed profile has discontinuities of the derivative in $x_{1}$ and $x_{2}$. The horizontal span of a bottom rise is $L=x_{2}-x_{1}$, its incline is 1 in $L /\left(h_{0}-h_{1}\right)$, or 1 in $L / 0.4$. Similar to the previous papers, we vary $L$ in order to obtain the reflection coefficient curves for the VBM and WKB - functions $R(L)$ in each case. The transmission coefficient is then trivially calculated and will not be given.

In addition, we make another set of simulations with fixed $L_{0}=6$ $\mathrm{m}$ (i.e. the slope is $1: 15)$ and varying $\omega$. This provides the reflection curves $R(\omega)$ for VBM and WKB with $\omega \in[0.2,4.5]$. Indeed, in the described layout there can be two parameters on which the reflection coefficient $R$ is dependent. Naturally, one is the incline of the slope, which we vary via the parameter $L$ in our simulations. And for the other parameter it is convenient to take the angular frequency of the incoming waves.

\subsubsection{Reflection in WKB-approximation}

Unlike in the VBM, the reflected signal (116) is already separated from the influxed signal in case of the reflection-capable WKB-approximation. Thus the reflection coefficient is obtained analytically.

When discussing the classical WKB-method for slowly varying bottom profile, it is common to use for $c(x)$ in $(76)$ the expression for shallow water waves: $c_{0}(x)=\sqrt{g h(x)}$. There is, however, another opportunity thanks to the fact that we simulate a sinusoidal signal. We also make use of the phase speed according to the exact dispersion at 


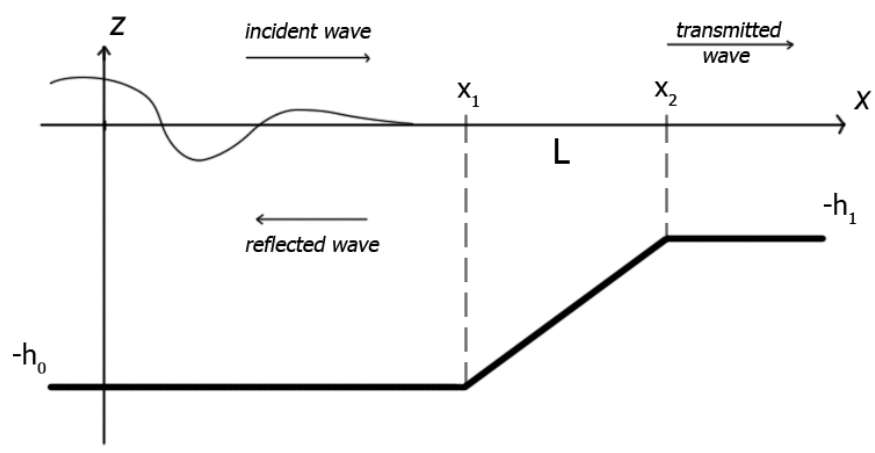

Figure 30: Schematic layout of the 'plane slope' experiment. Bottom profile is piecewise-linear and rises from $-h_{0}=-0.6 \mathrm{~m}$ to $-h_{1}=-0.2 \mathrm{~m}$ between $x_{1}$ to $x_{2}$. An incident wave coming from the left is partially reflected to the left and partially transmitted to the right.

varying bottom. To that end, for any fixed $\omega$, from the exact dispersion relation

$$
\omega=\Omega(\kappa(x), h(x))
$$

we determine the wave number $\kappa(x)$, dependent on the depth $h(x)$, and thus obtain the phase speed

$$
c(x)=\omega / \kappa(x) .
$$

In the derived above reflective WKB-model we use both $c_{0}(x)$ and $c(x)$ functions in expression (108), obtaining respectively functions $B_{0}(x)$ and $B(x)$. In order to compare, we use both of these to calculate the reflected wave according to (116), and we will show that the choice of the exact phase speed yields better results.

Having simulated the reflection according to the formula (116) for all slopes $L \in[0,12]$, we give the reflection coefficient curve in Fig. 31 . In the same figure we demonstrate the functions $B(x)$ and $B_{0}(x)$ for $L=8 \mathrm{~m}$, calculated according to (108) and used in the computations of $R(L)$.

Using the same WKB-reflection formula (116) we obtained the reflection curves $R(\omega)$ for $\omega \in[0.2,4.5]$ with fixed $L_{0}=6 \mathrm{~m}$ for both exact phase speed and shallow water approximation. They are shown in Figure 32. Notice that for small $\omega$ (long waves) the reflection coefficient is the same for both approximations, and for larger $\omega$ (shorter waves) the curves separate. This can be understood since for larger $\omega$ the deviation of the shallow water speed from the exact phase speed become larger. 

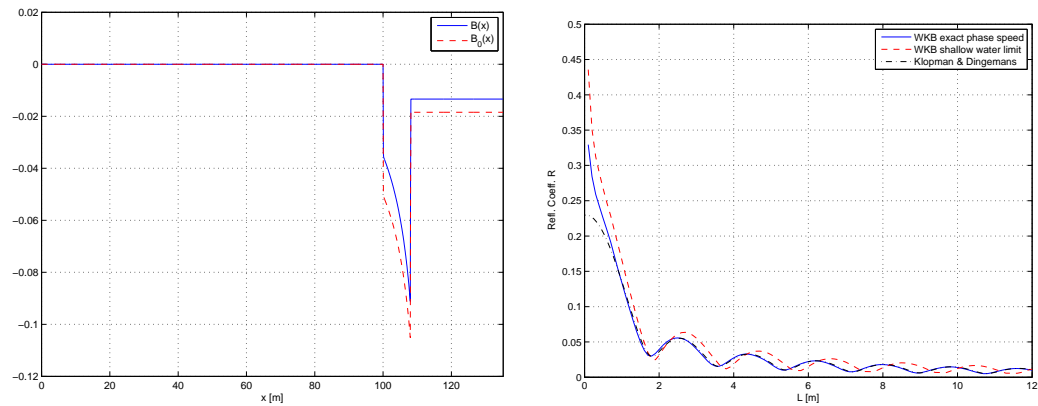

Figure 31: To the left are the functions $B(x)$ and $B_{0}(x)$ for $L=8 \mathrm{~m}$ : for the exact phase speed (solid) and for the shallow water limit (dashed). To the right the reflection coefficient curve $R(L)$ with fixed $\omega_{0}=\sqrt{g} \approx 3.13$ $\mathrm{rad} / \mathrm{s}$ of the WKB for the exact phase speed (solid), for the shallow water limit (dashed) and the best result of Klopman \& Dingemans (dash-dot) are compared.

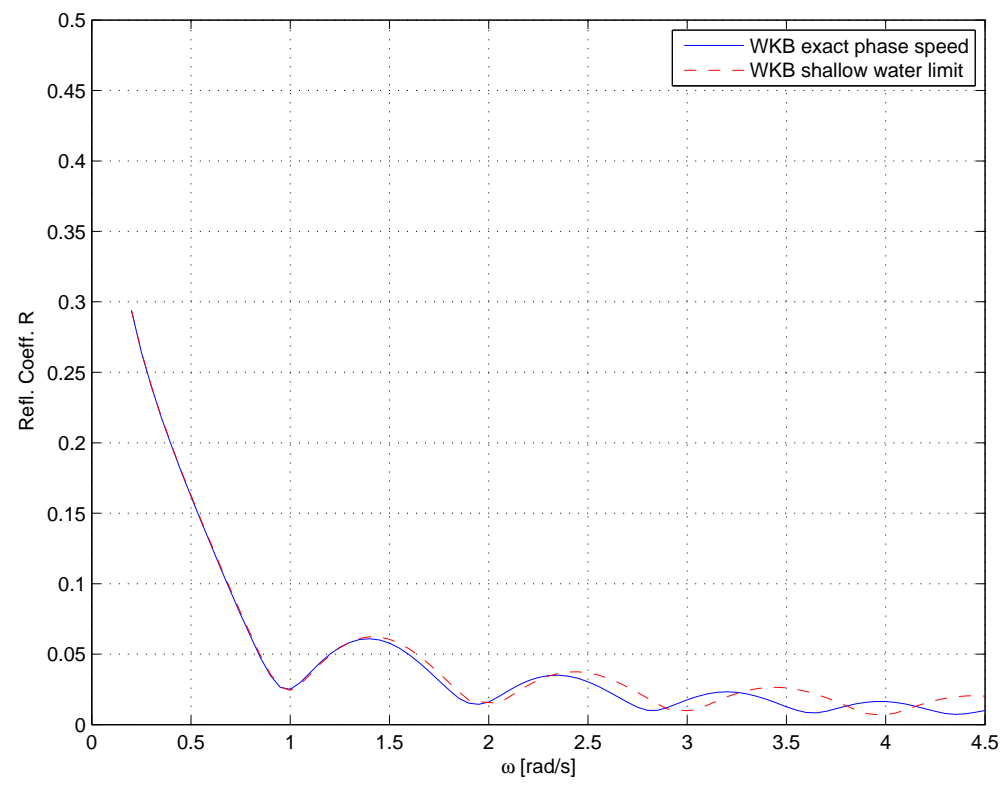

Figure 32: Reflection curves $R(\omega)$ with fixed $L_{0}=6 \mathrm{~m}$ of the WKB with the exact phase speed (solid) and for the shallow water limit (dashed) are compared. 


\subsubsection{Reflection in VBM}

As regards the reflected signal in VBM, the way to measure it is a bit more complicated.

At the zero point $x=0$ we generate sinusoidal waves (117) with help of the immersed boundary condition [18], i.e. forcing term which spans the segment $\left[0, x_{\gamma}\right]$, with $x_{\gamma}$ small comparing to $x_{1}$ (see Section 2.3 for details). For $x>x_{\gamma}$ the action of the forcing term vanishes almost completely. The wave running to the right is partially reflected from the rise in the bathymetry profile and then its superposition with the signal $\eta_{0}$ is measured in some point $x_{m}$ between $x_{\gamma}$ and $x_{1}$ and used for calculation of the reflection coefficient. The domain and the period of the experiment is chosen in such a way that the reflected wave has not come to the left boundary yet, but the frontal transient distortions of the wave already have passed the measurement point; the signal in this point $x_{m}$ indicates the stable mode afterwards.

The measured signal $\eta_{m}$ consists of the influxed wave $\eta_{0}$ and the reflected wave $\eta_{\text {refl }}$, running in opposite directions. Hence, the reflected wave can be calculated as the difference

$$
\eta_{r e f l}=\eta_{m}-\eta_{0}^{\prime}
$$

where $\eta_{0}^{\prime}$ is the same influxed harmonic as $\eta_{0}$, but with a shifted phase. It should be noted that the reflected wave is periodic, with a certain maximal amplitude $B$. The reflection coefficient is calculated as the ratio

$$
R=\frac{B}{A}=\frac{\max \eta_{r e f l}}{\max \eta_{0}^{\prime}}=\frac{\max \eta_{m}-A}{A} .
$$

The influxed and reflected waves have the same period but opposite directions (that is, partially forming a standing wave), so certain care should be taken in choosing $x_{m}$. Of course, it is more robust to take a segment for measurement of the maximum in (121) instead of a point. Even better way to obtain $\eta_{\text {refl }}$ is to repeat each experiment with the flat bottom and wide enough domain, in order to obtain original undisturbed signal $\eta_{0}^{\prime}$ as a difference between two experiments, and we employ this better approach.

We performed simulations on a sufficiently fine grid of $L \in[0,12]$, and obtained the reflection coefficient curve $R(L)$ with fixed $\omega_{0}=$ $\sqrt{g} \approx 3.13 \mathrm{rad} / \mathrm{s}$. Consider Fig. 33; here we provided the reflection curve for both VBM and WKB, compared to the best results by Klopman \& Dingemans. In addition, we performed simulations with fixed $L_{0}=6$ and varying $\omega$. The result, together with WKB reflection curve for exact speed, is shown in the same figure. 

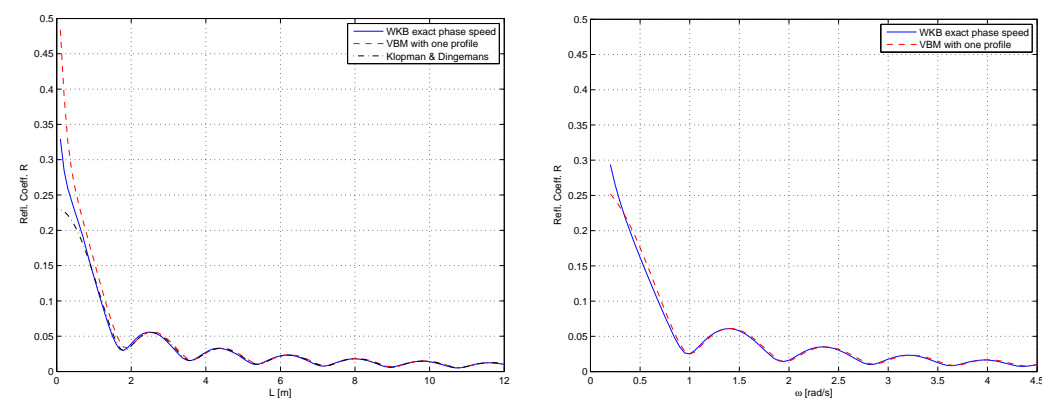

Figure 33: On the left: the reflection coefficient curves $R(L)$ with fixed $\omega_{0}=$ $\sqrt{g} \approx 3.13 \mathrm{rad} / \mathrm{s}$ of the reflection-capable WKB-approximation for the exact phase speed (solid), the linear VBM with one profile (dashed) and the best result of Klopman \& Dingemans (dash-dot). On the right: the reflection coefficient curves $R(\omega)$ with fixed $L_{0}=6$ $\mathrm{m}$ of the WKB-approximation (solid) and the VBM (dashed).

\subsection{CONCLUSIONS AND REMARKS}

The reflection curve by Klopman \& Dingemans [11], depicted in Figure 33, is almost identical to the accurate result by Porter \& Porter [17]. The result is obtained, however, with considerable effort by Klopman \& Dingemans, by deriving the time-harmonic VBM and tuning the normalization in the dispersive vertical profile of the VBM.

In contrast, our approach is straightforward: the model is used as-is, with the default normalization and the possibility of simulating wide range of waves. For example, the same model is employed in the article by Adytia \& van Groesen [3] to model irregular waves evolving over a slope. The model is easily adopted for $2 \mathrm{D}$ and nonlinearity, see e.g. $[2,3,12]$

Being simpler, our simulation provides resulting reflection curve of the VBM which looks almost identical to the reference for the slopes with $L>1 \mathrm{~m}$, diverging somewhat on more extreme, steeper slopes with $L<2 \mathrm{~m}$.

The same is suprisingly true for the derived reflection-capable WKBapproximation, which is analytic in its nature and provides an integral expression for the reflected wave signal. The reflection curve is very close to the reference for $L>1 \mathrm{~m}$. This model can be enhanced further by enabling more terms in the expansion (100).

In addition to the comparison with Klopman \& Dingemans, we made extra simulations and varied the parameter $\omega$ for fixed $L$. This demonstrated closeness of the reflection coefficient by the VBM and the reflective WKB-approximation. 

[1] N. Booij, A note on the accuracy of the mild-slope equation, Coastal Engineering 7 (1983) 191-203.

[2] D. Adytia, E. van Groesen, The Variational 2D Boussinesq model for wave propagation over a shoal, in: International Conference on Developments in Marine CFD, 18 - 19 November 2011, Chennai, India.

[3] D. Adytia, E. van Groesen, Optimized Variational 1D Boussinesq modelling of coastal waves propagating over a slope, Coast. Eng. 64 (2012) 139-150.

[4] M. Dingemans, Water wave propagation over uneven bottoms (World Scientic) 1 \& 2 (1997).

[5] E. van Groesen, Jaap Molenaar, Continuum Modeling in the Physical Sciences, in: Mathematical Modeling and Computation (SIAM) 13 (2007), 236 pp.; ISBN-13: 978-o-898716-25-2.

[6] E.van Groesen, Andonowati, Variational derivation of KdV-type of models for surface water waves, Phys. Lett. A 366 (2007) 195201.

[7] E. van Groesen, Andonowati, L. She Liam, I. Lakhturov, Accurate modelling of uni-directional surface waves, J. Comput. Appl. Math. 234 (2010) 1747-1756.

[8] E. van Groesen, Andonowati, Fully dispersive dynamic models for surface water waves above varying bottom, Part 1: Model equations, Wave Motion 48 (2011) 658-667.

[9] E. van Groesen, I. van der Kroon, Fully dispersive dynamic models for surface water waves above varying bottom, Part 2: Hybrid spatial-spectral implementations, Wave Motion 49 (2012) 198-211.

[10] G. Klopman, M. W. Dingemans, E. van Groesen, Propagation of wave groups over bathymetry using a variational Boussinesq model, in: Proceedings of the International Workshop on Water Waves and Floating Bodies, 2007.

[11] G. Klopman, M. W. Dingemans, Reflection in variational models for linear waves, Wave Motion 47 (2010) 469-489.

[12] G. Klopman, E. van Groesen, M. W. Dingemans, A variational approach to Boussinesq modelling of fully nonlinear water waves, J. Fluid Mech. 657 (2010) 36-63. 
[13] W. Kristina, O. Bokhove, E. van Groesen, Effective Coastal Boundary Conditions for Dispersive Tsunami Propagation, In preparation.

[14] I. Lakhturov, E. van Groesen, Optimized Variational Boussinesq Modelling; part 1: Broad-band waves over flat bottom, Internal report, University of Twente, The Netherlands. See http://eprints.eemcs.utwente.nl/.

[15] I. Lakhturov, D. Adytia, E. van Groesen, Optimized Variational ID Boussinesq Modelling for broad-band waves over flat bottom, Wave Motion 49 (2012) 309-322.

[16] E. Pelinovsky, Hydrodynamics of tsunami waves, Waves in Geophysical Fluids, in: CISM Courses and Lectures (Springer) 489 (2006) $1-48$.

[17] R. Porter, D. Porter, Approximations to the scattering of water waves by steep topography, J. Fluid Mech. 562 (2006) 279-302.

[18] L. She Liam, D. Adytia, and E. van Groesen, On internal wave generation for 1 - and $2 \mathrm{D}$ dispersive surface wave models, In preparation. 
In this thesis the Variational Boussinesq Model (VBM) was presented, and its applicability to modelling of complex wave fields was discussed. Numerical experiments for a few complicated test cases were performed, and compared to the data of the real-life experiments. The simulations were also compared with the results by other existing numerical codes, such as MIKE, SWASH and the AB-model, the later was briefly described in this thesis as well. In addition, specifically for needs of the reflection and run-up simulation an analytic model of the WKB (Wentzel-Kramers-Brillouin) type was derived and used in the previous chapter.

General advantages of the VBM were briefly mentioned in Chapter 2; here we briefly summarize the research done in this thesis, give a few recommendations and highlight possible directions for future advancements.

Dispersion in the VBM. The real-world applications provide requirements for improvements of a wave model. Being very flexible, the VBM allows for improvements in many respects. First of all, in this thesis we aim at the enhancement of dispersive characteristics of the model. When applied for such complicated cases, as for waves with broad spectra, the accuracy of the most currently used wave models and numerical codes are too low to satisfy needs of applications. In our judgment, the path to the better predictability of the real-world data goes through the enhancement of the model's dispersion. Indeed, it can be useless to improve, for example, nonlinearity in a model, if it provides not-so-good dispersion. As shown in Chapter 4, for the quite extreme test case (the wave was almost breaking at the focussing point), nonlinearity still plays a minor role comparing to the interference effect due to the linear dispersion.

The potential for dispersion improvement in the VBM comes from the ability to approximate the kinetic energy via approximating the vertical flow profile. Three steps were done on this way. The first is according to the 'classical' approach to the VBM, which provides a parabolic vertical dispersion profile. It performs reasonably well for wave lengths larger than twice the water depth. Generally speaking, the parabolic VBM would satisfy needs of tsunami simulations up to the coastal zone, as mostly the long waves have the appropriate propagation speed.

However, when it comes to laboratory waves with broad spectra, waves over a deep sea or e. g. a monochromatic wave train of a specific 
(high) frequency, the second choice, the cosh VBM with one vertical profile demonstrates its superiority over the previous choice. The dispersion accuracy for the long waves stays approximately the same as in the parabolic VBM. But additionally, for one specific wave number $\kappa$ the model obtains the exact dispersion as derived in the Airy wave theory, including the phase speed and the group speed.

The capacity of the model to choose the vertical profile of the fluid potential was exploited in this thesis by determining the optimal parameter value $\kappa$ in a parameterized class of profiles. This parameter is an effective wave number, the potential profile of which is taken as an approximation of the potential profile of all other waves with different wave numbers. The optimal parameter was found from an interesting minimum kinetic energy principle that depends on properties of the influxed signal (an initial wave profile can be easily used as well). Hence, in contrast with most other wave models, optimal dispersive properties are determined before the model is specified for simulating the evolution.

For applications, where the wave spectrum is very broad, even performance of the optimized cosh VBM with one vertical profile can become too poor. This is not a rare case: for example, for waves having the realistic JONSWAP spectrum the wave length in the spectral tail can become several times smaller than in the spectral peak. As demonstrated in Chapter 3, such short waves can become important for the appropriate focussing of a wave train, adding up to $15-20 \%$ of the wave amplitude.

For such extreme test cases the third choice, presented in Chapter 4, the optimized VBM with multiple cosh-vertical profiles shows really good performance. Already with two vertical profiles the dispersion accuracy errors become small enough to allow for almost exact reproduction of the real-life wave shape and amplitude. This can be done partially thanks to the appropriate choice of the parameters. For the effective wave numbers $\kappa_{j}$-s, used in this type of VBM, Airy theory provides the exact dispersion. Using the minimum kinetic energy principle it was shown that this criterium leads to wave numbers $\kappa_{j}$-s in an optimal way, minimizing the kinetic energy error, simultaneously minimizing the error in phase and group speed. Again, to determine the optimal values we used properties of the influxed signal (or of an initial wave profile); this then determines the specific dispersive properties used in the simulation.

Of course, additional accuracy does not come without a price. Each profile in the superposition of vertical dispersion profiles adds an addition equation to the elliptic PDE system, which has to be solved at each time step in the numerical code. Although the performance of the $1 \mathrm{D}$ VBM with 2 vertical profiles is quite fast $(3-5$ seconds to compute 1 second of the modelling time; straightforward FEM implementation in 
MATLAB), for $2 \mathrm{D}$ simulations this can become insufficient. Such types of simulations include modelling of harbour waves, tsunami run-up, coastal zone modelling. It can be recommended using and investigating the performance of fast iterative solvers with preconditioning for the solution of the elliptic PDE subsystem, and trying out different solvers for the time stepping.

Kinetic energy optimization. As said above, by minimizing the kinetic energy error one chooses the optimal parameters of the model, simultaneously minimizing the error in phase and group speed. This, in turn, leads to the very accurate simulation results, especially for the VBM with multiple profiles. This novel kinetic energy criterium is very natural for the VBM, and we would encourage its use, when a spectrum of an influxed signal (or an initial wave profile) is known. Then the optimal parameters of the model can be pre-calculated, thus, such improvement will not slow down the main computational loop.

It would be also interesting to investigate possibilities of introducing similar optimization criteria into different wave models.

Reflection. In Chapter 5 both the linear cosh VBM and the derived analytic WKB-type approximation demonstrated very good reflection performance, especially for the not-too-extreme inclines. This qualifies the VBM as a useful tool for tsunami run-up and harbour simulations, together with other coastal applications. It certainly would be interesting to investigate the reflective properties of the VBM and the WKBmodel for more complicated influxed waves and bathymetries.

Influxing boundary condition. The signalling problem naturally appears in such applications as simulations of the laboratory waves. Hence, it is important to introduce decent influx in a wave model. Wave generation in this thesis, both for the VBM and the AB-equation, was done using embedded (internally forced) influxing. This forcing (or source) term is calculated based on the analytic dispersion of the model and introduced into one of the equations in the VBM system, or added to the right hand side of the AB-equation. To our experience, this is the best way of generating a complex influx in the VBM, and especially, in the spectral setting of the AB numerical code.

It is tempting to see, whether (and how) such boundary condition could be introduced into different dispersive models.

Outflow boundary condition. Although test cases in this thesis did not require direct use of the outflow (or radiations) boundary conditions, their importance for the industry can not be underestimated. It would be interesting to investigate how well Sommerfeld non-reflective boundary conditions perform, how good is the standard absorption or damping layer for the VBM, and certainly, how to tune up parameters of such boundary conditions for the optimal performance of the model. 
Another question of interest is whether above-mentioned forcing term approach can be adjusted for using as a radiation boundary condition.

Numerical implementation. This thesis concentrates mostly on the modelling process. Although some details of the numerics are provided, the area for more deep analysis of the numerical VBM schemes is open for research. Many issues can be investigated and addressed, from analysis of numeric errors (one such example is in Subsection 2.1.5) and to optimization of a numerical implementation speed (this would be more needed in two-dimensional VBM). 


\section{APPENDIX}

Here we provide some secondary details as regards the FEM numerical implementation of the VBM, including the coefficients $\alpha, \beta, \gamma$, and the matrices used in the numerical code. The kinetic energy principle, used in Chapters 3 and 4 , is applied to JONSWAP spectra in order to observe the dependence of the optimal wave number on a spectrum's maximum.

\subsection{VAlues of COEFFicients $\alpha, \beta$ AND $\gamma$}

In Section 3.2 we have provided the coefficients $\alpha, \beta$ and $\gamma$ for the one-profile VBM, both for the parabolic- and cosh-approximation. In Chapter 4 , however, we have not shown the exact coefficients expressions.

Using the notation $H$ for the full depth until the surface, $H=h+\eta$, these integral coefficients are calculated as:

$$
\begin{aligned}
\alpha_{j j}\left(\kappa_{j}\right)= & -\frac{H}{2} \tanh ^{2} \kappa_{j} H-\frac{3}{2 \kappa_{j}} \tanh \kappa_{j} H+\frac{3}{2} H, \\
\alpha_{j m}\left(\kappa_{j}, \kappa_{m}\right)= & H-\frac{1}{\kappa_{j}} \tanh \kappa_{j} H-\frac{1}{\kappa_{m}} \tanh \kappa_{m} H+ \\
& \frac{1}{2\left(\kappa_{j}+\kappa_{m}\right)}\left[\tanh \kappa_{j} H+\tanh \kappa_{m} H\right]+ \\
& \frac{1}{2\left(\kappa_{j}-\kappa_{m}\right)}\left[\tanh \kappa_{j} H-\tanh \kappa_{m} H\right], \text { for } j \neq m, \\
\beta_{j}\left(\kappa_{j}\right)= & \frac{1}{\kappa_{j}} \tanh \kappa_{j} H-H, \\
\gamma_{j j}\left(\kappa_{j}\right)= & \frac{\kappa_{j}^{2} H}{2} \tanh \kappa_{j} H+\frac{\kappa_{j}}{2} \tanh \kappa_{j} H-\frac{\kappa_{j}^{2} H}{2}, \\
\gamma_{j m}\left(\kappa_{j}, \kappa_{m}\right)= & \frac{\kappa_{j} \kappa_{m}}{2\left(\kappa_{j}+\kappa_{m}\right)}\left[\tanh \kappa_{j} H+\tanh \kappa_{m} H\right]- \\
& \frac{\kappa_{j} \kappa_{m}}{2\left(\kappa_{j}-\kappa_{m}\right)}\left[\tanh \kappa_{j} H-\tanh \kappa_{m} H\right], \text { for } j \neq m .
\end{aligned}
$$

Particularly, for 1-profile approximation this yields

$$
\begin{aligned}
& \alpha_{11}\left(\kappa_{1}\right)=-\frac{H}{2} \tanh ^{2} \kappa_{1} H-\frac{3}{2 \kappa_{1}} \tanh \kappa_{1} H+\frac{3}{2} H, \\
& \beta_{1}\left(\kappa_{1}\right)=\frac{1}{\kappa_{1}} \tanh \kappa_{1} H-H, \\
& \gamma_{11}\left(\kappa_{1}\right)=\frac{\kappa_{1}^{2} H}{2} \tanh ^{2} \kappa_{1} H+\frac{\kappa_{1}}{2} \tanh \kappa_{1} H-\frac{\kappa_{1}^{2} H}{2} .
\end{aligned}
$$


The Taylor expansion of these coefficients around $\kappa_{1}=0$ for 1 -profile approximation is given by:

$$
\begin{aligned}
& \alpha_{11}\left(\kappa_{1}\right)=H\left[\frac{2}{15}\left(\kappa_{1} H\right)^{4}-\frac{34}{315}\left(\kappa_{1} H\right)^{6}+O\left(\left(\kappa_{1} H\right)^{8}\right)\right], \\
& \beta_{1}\left(\kappa_{1}\right)=H\left[-\frac{1}{3}\left(\kappa_{1} H\right)^{2}+\frac{2}{15}\left(\kappa_{1} H\right)^{4}-\frac{17}{315}\left(\kappa_{1} H\right)^{6}+O\left(\left(\kappa_{1} H\right)^{8}\right)\right], \\
& \gamma_{11}\left(\kappa_{1}\right)=\frac{1}{H}\left[\left(\kappa_{1} H\right)^{4}-\frac{4}{15}\left(\kappa_{1} H\right)^{6}+O\left(\left(\kappa_{1} H\right)^{8}\right)\right],
\end{aligned}
$$

The Taylor expansion for the 1-profile approximation around $k=0$ yields

$$
\begin{gathered}
\Omega_{V B M} \approx c_{0} k\left[1-\frac{\beta_{1}^{2}}{2 H \gamma_{11}} k^{2}+\left(\frac{\alpha \beta_{1}^{2}}{2 H \gamma_{11}^{2}}-\frac{\beta_{1}^{4}}{8 H^{2} \gamma_{11}^{2}}\right) k^{4}-\right. \\
\left.\left(\frac{\alpha_{11}^{2} \beta_{1}^{2}}{2 H \gamma_{11}^{3}}-\frac{\alpha_{11} \beta_{1}^{4}}{4 H^{2} \gamma_{11}^{3}}+\frac{\beta_{1}^{6}}{16 H^{3} \gamma_{11}^{3}}\right) k^{6}+O\left(k^{8}\right)\right] .
\end{gathered}
$$

It should be remarked that these expressions are valid for a VBM with any vertical potential approximation $F(z)$ in (64), although the integral coefficients (68) can depend on model parameters, e.g. the wave number $\kappa_{1}$ as appears in the approximation above. It can be noted also that after substituting the cosh-coefficients values (68) into (124) the Taylor expansion around $k=0$ yields the same expression as in the parabolic case, see Klopman e.a. [2, 3] and Madsen e.a. [4, 5].

\subsection{MATRICES IN VBM NUMERICAL IMPLEMENTATION}

In Chapter 2 we have described the numerical implementation of the VBM. Here we add some details about the matrices used in the FEM numerical code.

For the matrices we make use of the upper index, denoting spatial distribution of weights. In particular, the so-called mass matrix $M$ is comprised of the elements $M_{i, j}^{\rho}=\int \rho(x) T_{i}(x) T_{j}(x) d x$, where $\rho$ represents certain weight of the element. For instance, the depth function $h(x)$ can be used as the weight; the upper index is omitted for the unitary weight. The so-called stiffness matrix $D$ consists of the elements $D_{i, j}^{\rho}=\int \rho(x) T_{i}^{\prime}(x) T_{j}^{\prime}(x) d x$.

In the numerical system, corresponding to the simplest case (15) of the linear VBM with one profile, no upper indices are used, i. e. all weights are unitary. For the one horizontal dimensions both matrices $M$ and $D$ are trilinear. The diagonal elements of the mass matrix are:

$$
m_{k k}=\frac{1}{3}\left(\triangle x_{k-1}+\triangle x_{k}\right),
$$

except for corners, where $m_{11}=\frac{1}{3} \triangle x_{1}$ and $m_{n n}=\frac{1}{3} \triangle x_{n-1}$. Here we denote the grid step between $k$-th and $(k+1)$-th point as $\triangle x_{k}$. The elements above and below the main diagonal are: 


$$
m_{k, k-1}=\frac{1}{6} \triangle x_{k-1}, \quad m_{k, k+1}=\frac{1}{6} \triangle x_{k} .
$$

On the diagonal of the stiffness matrix there are elements:

$$
d_{k k}=\frac{1}{\triangle x_{k-1}}+\frac{1}{\triangle x_{k}}
$$

except for corners, where $d_{11}=\frac{1}{\triangle x_{1}}$ and $d_{n n}=\frac{1}{\triangle x_{n-1}}$. Above and below the main diagonal there are elements:

$$
d_{k, k-1}=-\frac{1}{\triangle x_{k-1}}, \quad d_{k, k+1}=-\frac{1}{\triangle x_{k}} .
$$

Suppose, the grid is equidistant with the grid step $\triangle x$. Then the matrices $M$ and $D$ have the simplest form:

$$
M=\frac{\Delta x}{6}\left(\begin{array}{ccccccc}
2 & 1 & 0 & 0 & \cdots & & 0 \\
1 & 4 & 1 & 0 & & & \\
0 & 1 & 4 & 1 & & & \\
0 & 0 & 1 & 4 & & & \vdots \\
\vdots & & & & \ddots & & 0 \\
& & & & & 4 & 1 \\
0 & & & \cdots & 0 & 1 & 2
\end{array}\right)
$$

and

$$
D=\frac{1}{\Delta x}\left(\begin{array}{ccccccc}
1 & -1 & 0 & 0 & \ldots & & 0 \\
-1 & 2 & -1 & 0 & & & \\
0 & -1 & 2 & -1 & & & \\
0 & 0 & -1 & 2 & & & \vdots \\
\vdots & & & & \ddots & & 0 \\
& & & & & 2 & -1 \\
0 & & & \ldots & 0 & -1 & 1
\end{array}\right) .
$$

When the matrices have to be calculated with certain weights, this does not complicate the calculation procedure much. The new matrices $M_{\gamma}, D_{\alpha}$, etc. are obtained from the presented above matrices $M$ and $D$ by multiplying each row $j$ with the values $\gamma_{j}$ and $\alpha_{j}$, respectively; these values are calculated in the grid-point $x_{j}$.

\subsection{JONSWAP SPECTRUM AND KINETIC ENERGY MINIMIZATION}

In Chapters 3 and 4 we derived the novel kinetic energy optimization criterion, with which we successfully simulated the complicated cases 
of the focussing wave groups. In the first place we observed the interesting fact that the optimal wave number (in case of the one-profile VBM), calculated according to this criterion, is not even closely corresponding to the peak frequency in the spectrum. This is contradictory to the first guess we could make, but after some thought it becomes visible that not all wave numbers of the spectrum are equally important to the kinetic energy minimization problem. Apparently, shorter waves, though not dominant in the spectrum, provide more input for it. This is due to the inevitably larger error VBM dispersion relation has for the shorter waves.

In a sea the waves are usually generated by wind. There is a number of spectra estimates for realistic wave fields developed fully or partially in the sea. Here we use one of them, the so-called JONswAP spectrum [1], which represents the realistic spectrum of a wave field, according to the data collected in the North Sea.

The expression for the JONSWAP spectrum is

$$
S(\omega)=\frac{\alpha g^{2}}{\omega^{5}} \exp \left[-\frac{5}{4}\left(\frac{\omega_{p}}{\omega}\right)^{4}\right] \gamma^{r},
$$

where

$$
r=\exp \left[-\frac{\left(\frac{\omega}{\omega_{p}}-1\right)^{2}}{2 \sigma^{2}}\right] .
$$

Here $\omega_{p}$ is the peak (angular) frequency, connected in a standard way to the peak period $T_{p}, \omega_{p}=2 \pi / T_{p}$. The intensity of the spectrum $\alpha$ affects the total energy; we take $\alpha=0.0081$. The skewness parameter $\sigma$ defines the left- and right-sided widths: $\sigma=0.07$ for $\omega \leqslant \omega_{p}$ and $\sigma=0.09$ for $\omega>\omega_{p}$. The "peak enhancement" factor $\gamma$ is what differs the JonswaP spectrum from another well-known Pierson-Moskowitz spectrum [6]; we take $\gamma=3.3$. For calculations we use the fixed significant wave height $H_{m 0}=3 \mathrm{~m}$.

While varying the peak frequency $\omega_{p}$ of JonswAP spectra, we use the kinetic energy criterium (74) for calculations of the optimal frequency $v_{1}$ for the one-profile VBM with cosh-profile. In Figure 34 we plot two JONswAP spectra for the peak periods $T_{p}=9 \mathrm{~s}$ and $T_{p}=12$ s. Also in the same figure one can see the optimal frequency dependence on the spectral peak frequency for three different depths. Note, although the spectrum itself does not depend on depth, the optimal frequencies do depend on depth. We can observe that the optimal frequency is almost two times greater than the peak frequency of the spectrum. 

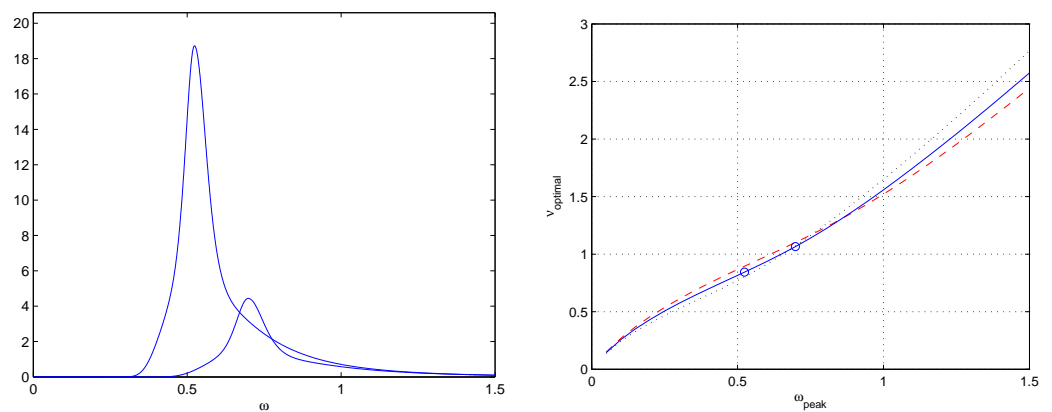

Figure 34: On the left: Jonswap spectra with the peak periods $T_{p}=9 \mathrm{~s}$ and $T_{p}=12 \mathrm{~s}$. On the right: optimal frequency $v_{1}$ for the one-profile VBM, depending on the peak frequency $\omega_{p}$. Three curves correspond to different depths: $20 \mathrm{~m}$ (dashed), $30 \mathrm{~m}$ (solid) and $50 \mathrm{~m}$ (dotted). Two circles show the spectral optima for the peak periods $T_{p}=9 \mathrm{~s}$ and $T_{p}=12 \mathrm{~s}$. The significant wave height $H_{m 0}$ in all cases is fixed and equal to $3 \mathrm{~m}$. 

[1] K. Hasselmann, T. Barnett, E. Bouws, H. Carlson, D. Cartwright, K. Enke, J. Ewing, H. Gienapp, D. Hasselmann, P. Kruseman, A. Meerburg, P. Mller, D. Olbers, K. Richter, W. Sell, H. Walden, Measurements of wind-wave growth and swell decay during the Joint North Sea Wave Project (JonswaP), Ergäzungsheft zur Deutschen Hydrographischen Zeitschrift Reihe A 8 (1973), 9-95.

[2] G. Klopman, M. W. Dingemans, E. van Groesen, A variational model for fully non-linear water waves of Boussinesq type, in: Proceedings of the International Workshop on Water Waves and Floating Bodies, 2005.

[3] G. Klopman, M. W. Dingemans, E. van Groesen, Propagation of wave groups over bathymetry using a variational Boussinesq model, in: Proceedings of the International Workshop on Water Waves and Floating Bodies, 2007.

[4] P.A. Madsen, R. Murray, O. R. Sørensen, A new form of the Boussinesq equations with improved linear dispersion characteristics, Coast. Eng. 15 (1991), 371-388.

[5] P.A. Madsen, O. R. Sørensen, A new form of the Boussinesq equations with improved linear dispersion characteristics. Part 2. A slowly-varying bathymetry, Coast. Eng. 18 (1992), 371-388.

[6] W. Pierson, L. Moskowitz, Proposed Spectral Form for Fully Developed Wind Seas Based on the Similarity Theory of S. A. Kitaigorodskii, J. Geophys. Res. 69 (1964), 5181-519o. 



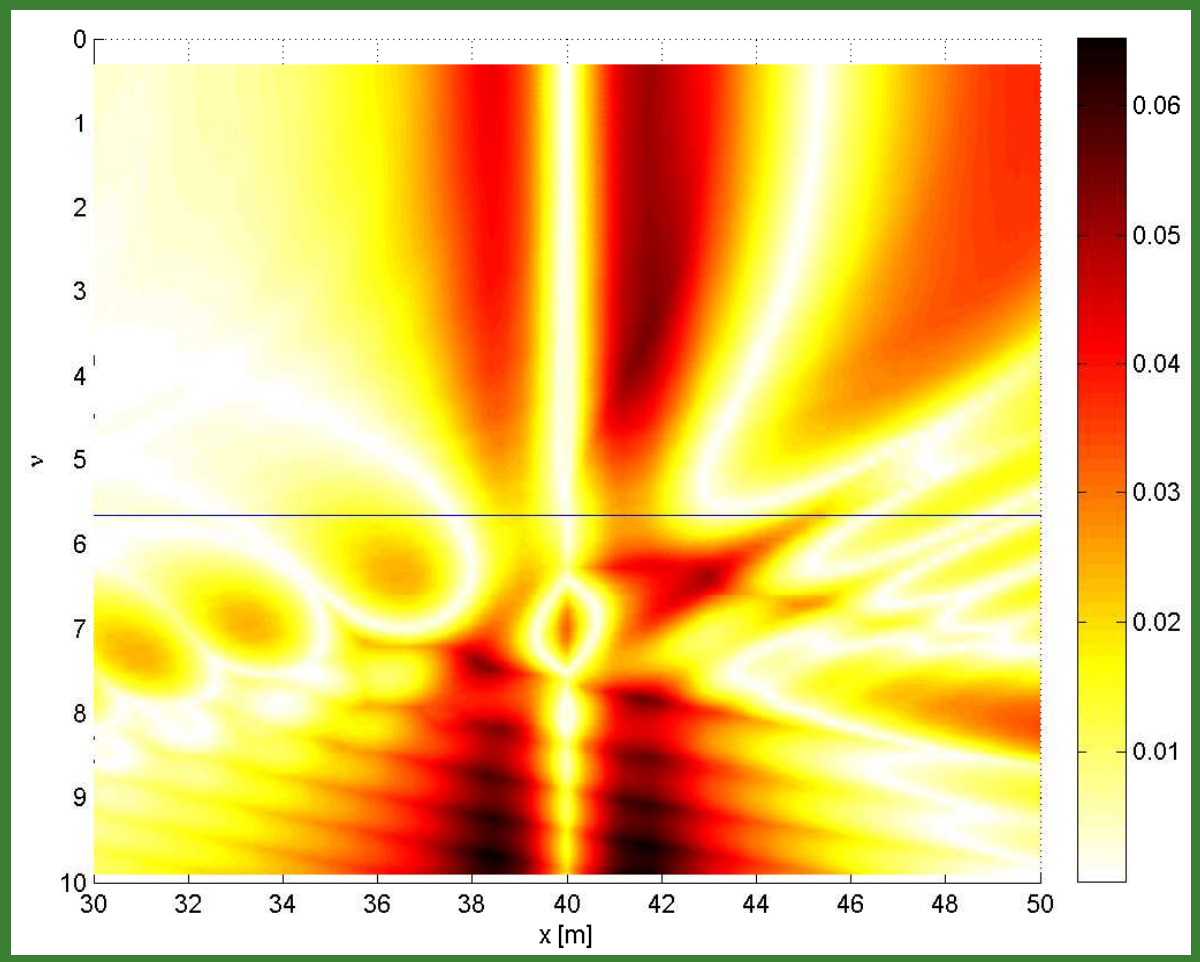

ISBN: 978-90-365-3447-5 